US Army Corps

of Engineers ${ }_{\circledast}$

Engineer Research and

Development Center

Installation Technology Transition Program

\title{
Evaluation of Roof Leak Detection Utilizing Unmanned Aircraft Systems Equipped with Thermographic Sensors
}

Samuel J. Vance, Matthew E. Richards, and

December 2018 Matthew C. Walters

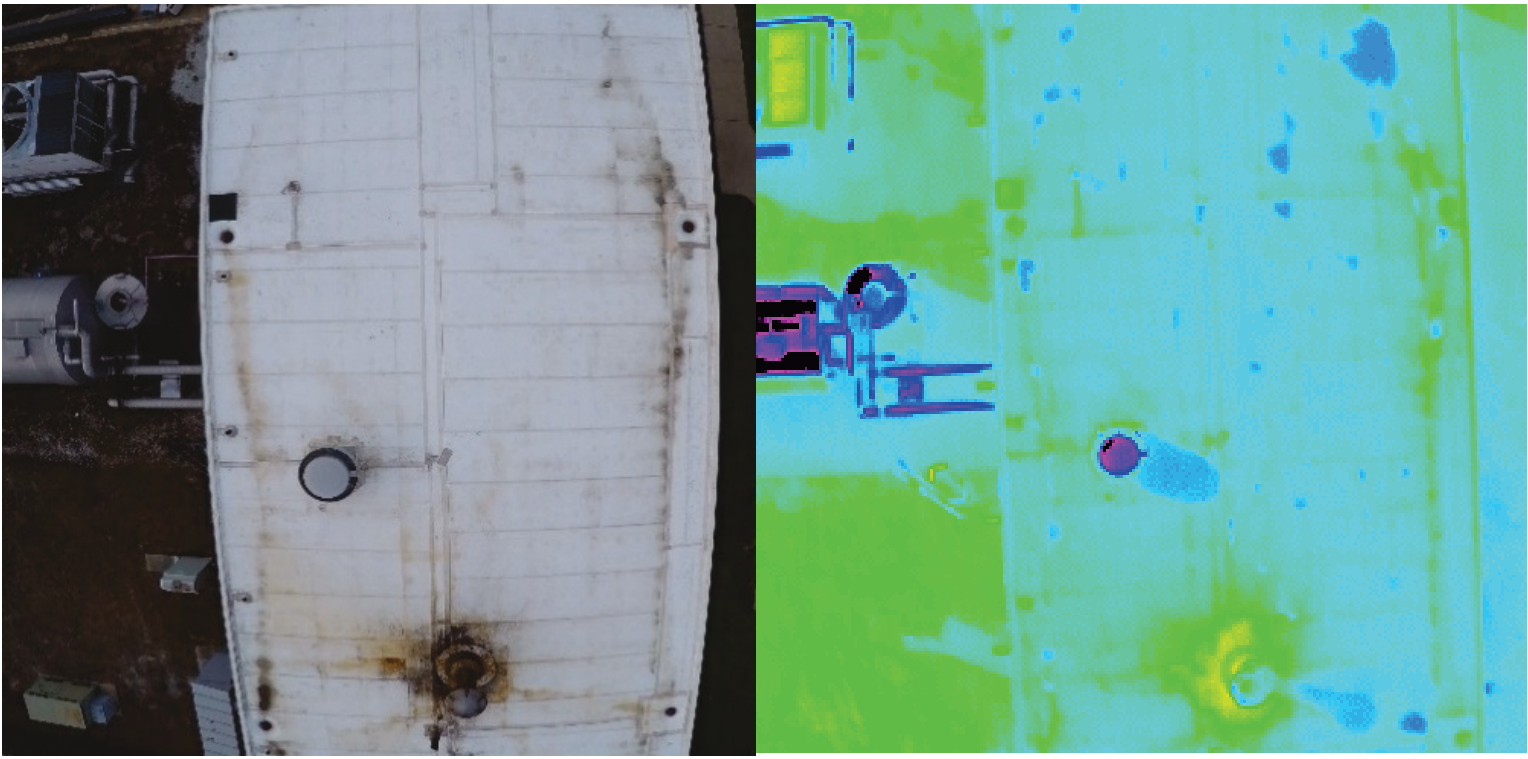


The U.S. Army Engineer Research and Development Center (ERDC) solves the nation's toughest engineering and environmental challenges. ERDC develops innovative solutions in civil and military engineering, geospatial sciences, water resources, and environmental sciences for the Army, the Department of Defense, civilian agencies, and our nation's public good. Find out more at www.erdc.usace.army.mil.

To search for other technical reports published by ERDC, visit the ERDC online library at http://acwc.sdp.sirsi.net/client/default. 


\section{Evaluation of Roof Leak Detection Utilizing Unmanned Aircraft Systems Equipped with Thermographic Sensors}

Samuel J. Vance, Matthew E. Richards, and Matthew C. Walters

Construction Engineering Research Laboratory

U.S. Army Engineer Research and Development Center

2902 Newmark Drive

Champaign, IL 61822

Final report

Approved for public release; distribution is unlimited.

Prepared for Office of the Assistant Chief of Staff for Installation Management (OACSIM) 600 Army Pentagon

Washington, DC 20314-1000

Under Project 457492, “Aerial Thermography Utilizing Autonomous Unmanned Aircraft Systems" 


\section{Abstract}

Management of the extensive U.S. Army roof portfolio increasingly relies on intelligent, data-driven decisions in a resource-limited environment. Roof leaks constitute millions of dollars in damage to equipment, insulation, and other assets, and can be the source for mold and other human environmental hazards. In recent years, thermographic imaging has been used as a nondestructive means to detect the location of wet insulation in low-pitched roofs. Traditional acquisition methods of nondestructive roof leak detection include handheld rooftop inspection and aerial imaging by conventional, manned aircraft. Through the advent and maturation of unmanned aircraft system (UAS) technology, nondestructive thermographic inspections are a viable alternative to traditional thermographic data acquisition methods. This report concludes that the use of a UAS for roof leak detection is a cost-effective and efficient alternative to other inspection methods, and it gives recommendations for the U.S. Army's use of the technology.

DISCLAIMER: The contents of this report are not to be used for advertising, publication, or promotional purposes. Citation of trade names does not constitute an official endorsement or approval of the use of such commercial products. All product names and trademarks cited are the property of their respective owners. The findings of this report are not to be construed as an official Department of the Army position unless so designated by other authorized documents.

DESTROY THIS REPORT WHEN NO LONGER NEEDED. DO NOT RETURN IT TO THE ORIGINATOR. 


\section{Contents}

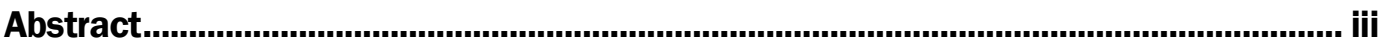

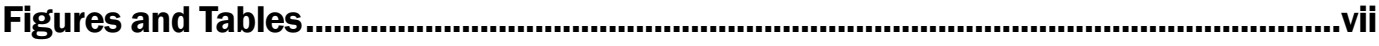

Preface

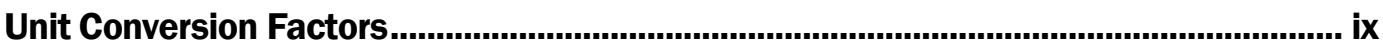

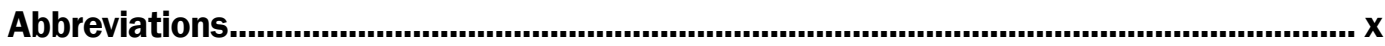

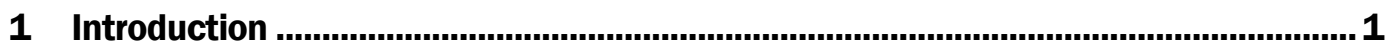

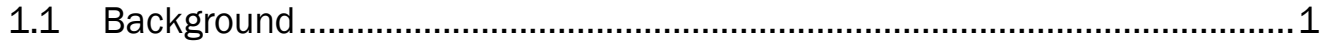

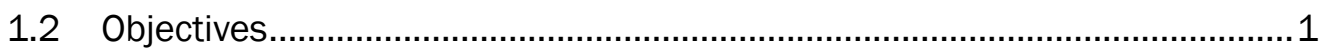

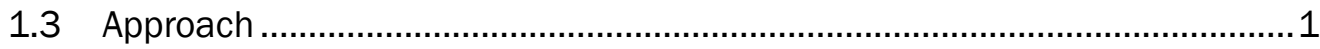

2 Roof Leak Detection Using Thermal Imaging............................................................. 3

2.1 Science of roof leak detection via thermal imaging ........................................

2.2 Traditional thermal roof imaging acquisition ..............................................

2.3 Unmanned aircraft system thermal imaging ............................................... 5

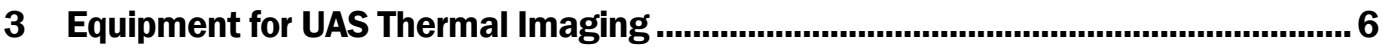

4 Assessments at ERDC-CERL

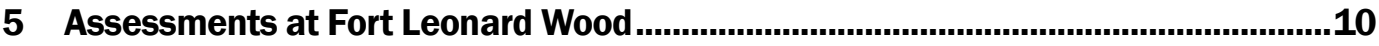

6 Comparison of Thermal Roof Leak Detection Methods and Feasibility for Use by Department of Army..............................................................................12

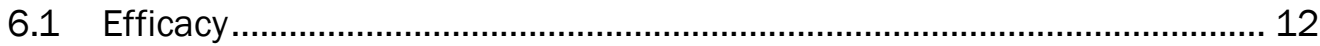

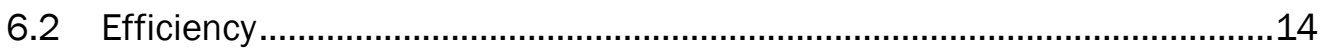

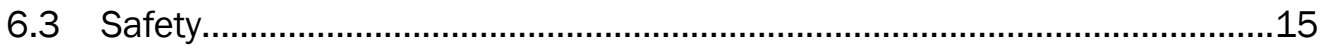

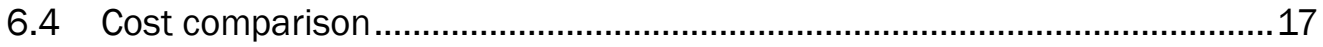

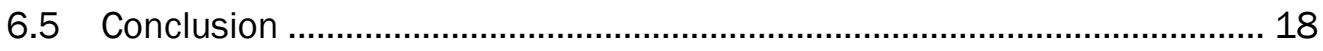

7 Outline of Implementation Plan for Army Installations...........................................19

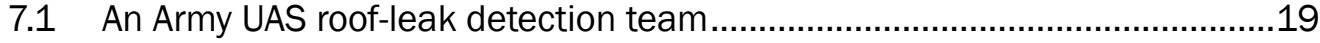

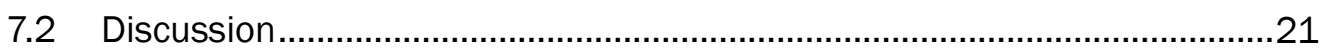

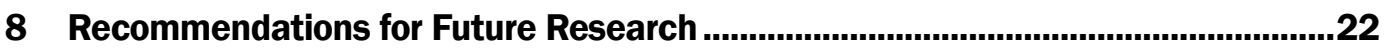

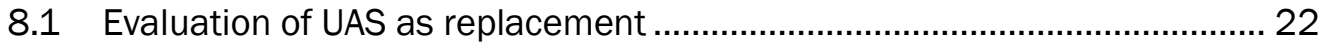

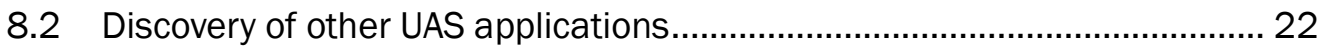

8.3 Development of UAS toolkit ................................................................. 22

9 Unmanned Aircraft System Flight Approval Process .............................................23

9.1 Standing operating procedure development ...............................................24 
9.2 System Safety Management Plan development........................................ 25

9.3 Operational Risk Acceptance Letter ........................................................ 25

9.4 Material Risk Acceptance Letter (UAS model-specific) .............................. 25

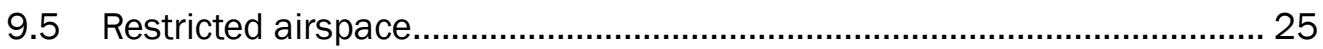

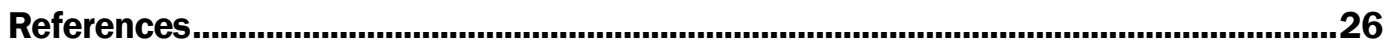

Appendix A: Arduino Source Code .................................................................................27

Appendix B: Raspberry Pi, Flir Camera Control Source Code ...............................................31

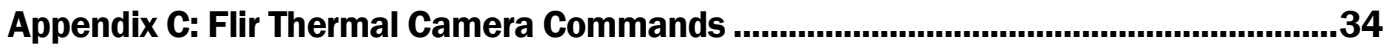

Appendix D: Standing Operating Procedure Template/Sample for Unmanned

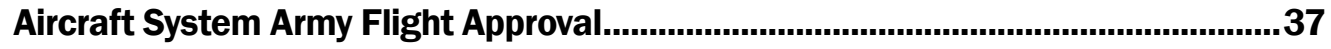

Appendix E: System Safety Management Plan Template/Sample ....................................79

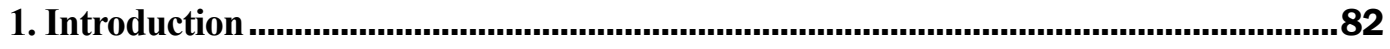

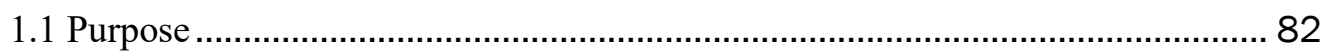

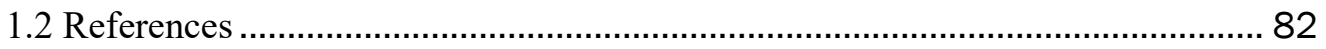

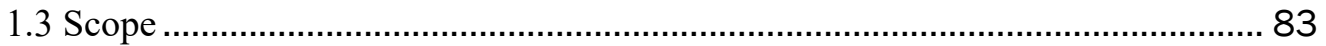

1.4 System Safety Program Goal................................................................ 83

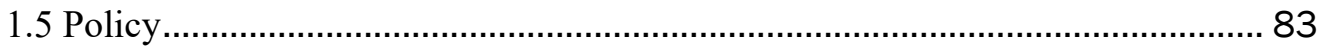

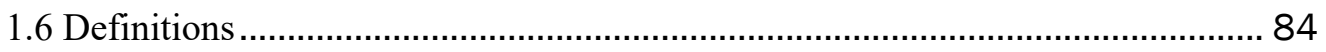

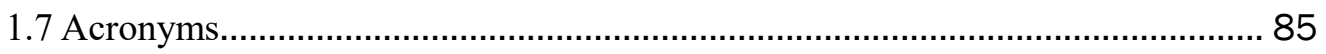

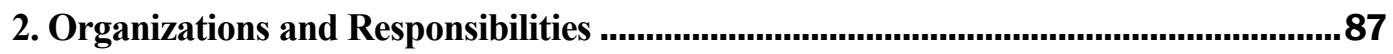

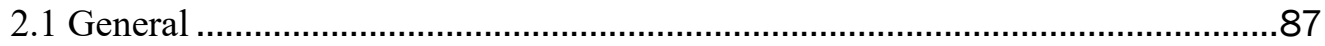

2.2 Integration of Associated Disciplines......................................................... 87

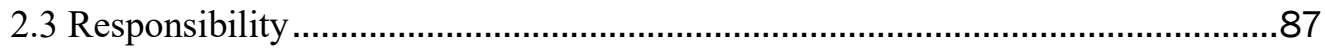

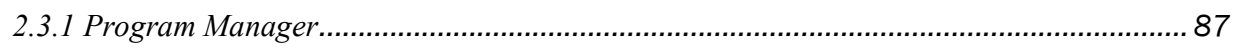

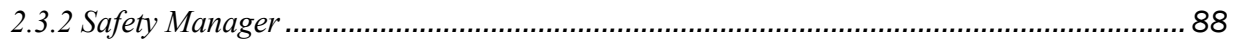

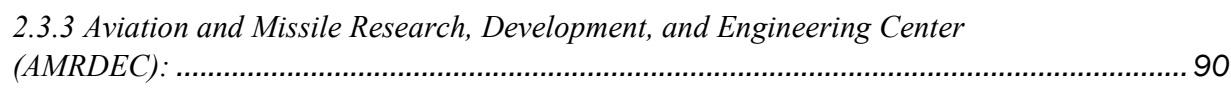

2.3.4 Contractor System Safety Support........................................................................ 90

3. Risk Management ..............................................................................................90

3.1 Hazard Severity Categories .................................................................... 90

3.2 Hazard Probability Levels .......................................................................91

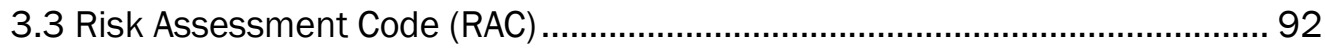

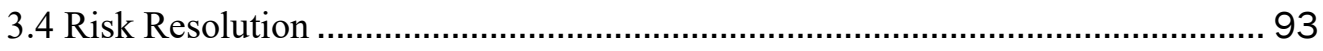

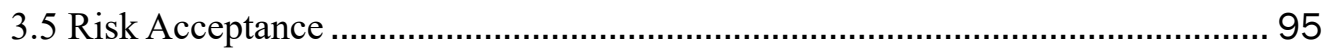

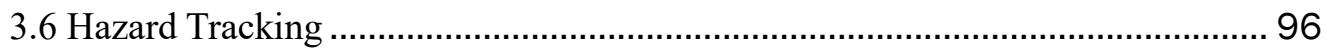

Appendix F: Operational Risk Assessment and Acceptance Memo Template

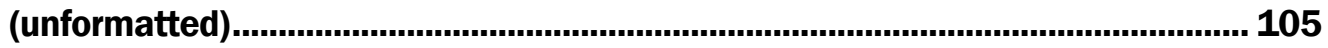


Appendix G: Material Risk Assessment Memo Template.......................................... 108 Report Documentation Page 


\section{Figures and Tables}

\section{Figures}

Figure 1. Relative temperatures of low-slope roofs as viewed through a thermal

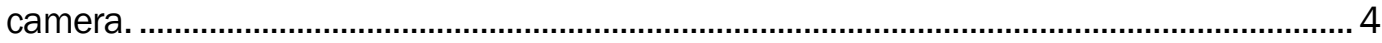

Figure 2. Location of Central Plant Facility on ERDC-CERL's campus. ................................. 8

Figure 3. Visual spectrum and thermal images of ERDC-CERL's Central Plant roof. ........... 9

Figure 4. Red circles indicate locations of two building rooftops assessed at Fort Leonard Wood quarry.

Figure 5. Northernmost quarry building's roof images (thermal image, left side; photograph, right side), with UAV positioned directly over roof).

Figure 6. Northernmost quarry building, thermal image roof (UAV positioned to the south of the facility).

Figure 7. Visual spectrum image of Building 750 (lower left), acquired by helicopter during the 2015 ERDC-CERL project.

Figure 8. Thermal spectrum image of Building 750 (lower left), acquired by helicopter during the 2015 ERDC-CERL project.

Figure 9. Thermal image of quarry facility (right side) obtained by UAS. ............................14

Figure 10. Frequency/consequence matrix............................................................................

Figure 11. Frequency of incident vs. consequence of incident occurrence. .......................17

\section{Tables}

Table 1. UAS equipment list. 6

Table 2. Cost estimate and comparison of thermal roof-leak detection techniques.

Table 3. Estimated cost of Army UAS thermal roof-leak detection team. 


\section{Preface}

This study was conducted for the Office of the Assistant Chief of Staff for Installation Management (OACSIM) under the Installation Technology Transition Program (ITTP), Project Number 457492, "Aerial Thermography Utilizing Autonomous Unmanned Aircraft Systems (UASs).” The technical monitor was Mr. Philip Columbus, OACSIM.

The work was performed by the Engineering Processes Branch (CFN) of the Facilities Division (CF), U.S. Army Engineer Research and Development Center, Construction Engineering Research Laboratory (ERDCCERL). At the time of publication, Ms. Kelly Moon was the ERDC ITTP Manager; Mr. Charles G. Schroeder was Chief, CEERD-CFN; Mr. Donald K. Hicks was Chief, CEERD-CF; and Mr. Kurt Kinnevan, CEERD-CZT, was the associated technical director. The Deputy Director of ERDC-CERL was Dr. Kirankumar Topudurti, and the Director was Dr. Lance D. Hansen.

A special thanks to Mr. Joe Mata and Mr. Jeff Walsh for their aid in coordinating flights at Fort Leonard Wood, MO.

The Commander of ERDC was COL Ivan P. Beckman, and the Director was Dr. David W. Pittman. 


\section{Unit Conversion Factors}

\begin{tabular}{|c|c|c|}
\hline Multiply & By & To Obtain \\
\hline degrees (angle) & 0.01745329 & radians \\
\hline miles (nautical) & 1,852 & meters \\
\hline pounds (mass) & 0.45359237 & kilograms \\
\hline square feet & 0.09290304 & square meters \\
\hline
\end{tabular}




\section{Abbreviations}

\begin{tabular}{|c|c|}
\hline Term & Meaning \\
\hline AAAR & Abbreviated Aviation Accident Report \\
\hline $\mathrm{AAE}$ & Army Acquisition Executive \\
\hline A\&E & ammunitions and explosives \\
\hline AED & automated external defibrillator \\
\hline AED & Aviation Engineering Directorate \\
\hline AMC & Army Materiel Command \\
\hline AMCOM & Aviation and Missile Command \\
\hline AMRDEC & Aviation and Missile Research, Development, and Engineering Center \\
\hline AR & Army Regulation \\
\hline ASAP & as soon as possible \\
\hline ATC & Air Traffic Control \\
\hline AWR & Airworthiness Release \\
\hline CHPPM & Center for Health Promotion and Preventive Medicine \\
\hline $\mathrm{COA}$ & Certificate of Authority/Authorization \\
\hline COTS & commercial off-the-shelf \\
\hline CPR & cardiopulmonary resuscitation \\
\hline CRC & Combat Readiness Center \\
\hline DA & Department of the Army \\
\hline DA-PAM & Department of the Army Pamphlet \\
\hline DART & Downed Aircraft Recovery Team \\
\hline DoD & Department of Defense \\
\hline DPW & Directorate of Public Works \\
\hline DRMO & Defense Reutilization and Marketing Office \\
\hline DVR & digital video recorder \\
\hline EPDM & ethylene propylene diene monomer \\
\hline $\begin{array}{l}\text { ERDC- } \\
\text { CERL }\end{array}$ & $\begin{array}{l}\text { Engineer Research and Development Center-Construction } \\
\text { Engineering Research Laboratory }\end{array}$ \\
\hline FAA & Federal Aviation Administration \\
\hline FM & field manual \\
\hline FO & Flight Operator \\
\hline FPV & first-person view \\
\hline GCS & Ground Control Station \\
\hline GPIO & general purpose input/output \\
\hline GPS & global positioning system \\
\hline GSA & General Services Administration \\
\hline $\mathrm{HC}$ & Hazardous Classification \\
\hline
\end{tabular}




\begin{tabular}{|c|c|}
\hline Term & Meaning \\
\hline $\mathrm{HD}$ & Hazard Division \\
\hline $\mathrm{HHA}$ & HEALTH Hazard Assessment \\
\hline HRED & Human Research and Engineering Directorate \\
\hline IMA & Independent Medical Assessor \\
\hline IPT & Integrated Product Team \\
\hline $\begin{array}{l}\text { MANPRIN } \\
T\end{array}$ & Manpower Personnel Integration \\
\hline MC & Mission Commander \\
\hline MIL-STD & Military Standard \\
\hline MOA & memorandum of agreement \\
\hline $\mathrm{MOI}$ & Memorandum of Instruction \\
\hline NOTAM & Notice to Airman \\
\hline NOV & Notice of Violation \\
\hline PM & Program Manager \\
\hline $\mathrm{PM} / \mathrm{D}$ & Program Manager/Director \\
\hline PEO & Program Executive Officer \\
\hline PPE & personal protective Equipment \\
\hline PPM & pulse position modulation \\
\hline PRAM & Preliminary Report of Aviation Mishap \\
\hline RAC & Risk Assessment Code \\
\hline $\mathrm{RC}$ & radio control \\
\hline RFP & Request for Proposal \\
\hline ROI & return on investment \\
\hline SAA & Satellite Accumulation Area \\
\hline SM & Safety Manager \\
\hline so & safety observer \\
\hline SOP & Standing Operating Procedure \\
\hline SSMP & System Safety Management Plan \\
\hline SSP & System Safety Program \\
\hline SSRA & System Safety Risk Assessment \\
\hline SSWG & System Safety Working Group \\
\hline SUAS & small unmanned aircraft system \\
\hline TRADOC & Training and Doctrine Command \\
\hline TCM & TRADOC Capabilities Manager \\
\hline UA & unmanned aircraft \\
\hline UAS & unmanned aircraft system \\
\hline USB & Universal serial bus \\
\hline USACE & U.S. Army Corps of Engineers \\
\hline
\end{tabular}




\section{Introduction}

\subsection{Background}

Management of the large Army facility portfolio relies on intelligent, datadriven decisions. Due to the consequence of roof failure, performing accurate roof leak detection and roof condition assessment is critical to the overall lifespan of each facility. Accurate roof leak detection is aided by the use of thermographic imaging (see Chapter 2). Thermographic images unveil distresses not evident by traditional visual and destructive roof inspection techniques. ${ }^{1}$ Thermal imaging cameras can be used by inspection teams on rooftops, by manned flights equipped with cameras, or by an unmanned aircraft system (UAS). Challenges to thermographic roof imaging include the safety and efficacy of technicians performing roof-top thermographic inspections or alternatively the high costs, detail, and weather considerations associated with manned flight imaging.

\subsection{Objectives}

This study was designed to accomplish the following objectives:

1. Produce a comparison of UAS thermal roof leak detection technology with non-UAS thermal inspection techniques by using economic analysis and engineering quality measures.

2. Develop the process of flight approvals for UASs for the purposes of roof inspection and other engineering data-acquisition purposes.

3. Provide an outline for an implementation plan by the Army on the use of UAS thermal roof inspections.

4. Communicate suggestions for future research (if any).

\subsection{Approach}

The study took place on building roofs at the Engineer Research and Development Center-Construction Engineering Research Laboratory (ERDC-CERL) in Champaign, Illinois, and at Fort Leonard Wood, Mis-

1 Destructive techniques include the use of core samples, moisture probes, and gravimetric analyses. 
souri. A commercial off the shelf (COTS) UAS was equipped with a thermal camera and operated over low-slope roofs. Leaks were found via visual inspection of the thermal images. The flight approval process was delineated by research and communication with the Army Aviation Engineering Directorate (AED). An economic analysis and a risk analysis were developed from the information gathered. Lessons learned from the workflow of roof leak detection-purposed UAS operations led to creation of a preliminary Army UAS Roof Leak Detection implementation plan, which is included in Chapter 7 . 


\section{Roof Leak Detection Using Thermal Imaging}

\subsection{Science of roof leak detection via thermal imaging}

When water is introduced into an area below the water-resistant membrane of a low-slope roof section, the wet area exhibits dissimilar thermal characteristics when compared with the surrounding, dry roof section. Wet areas differ thermally from dry areas due to a higher composite thermal capacitance than surrounding dry areas. If viewed during a warming period, wet areas appear cooler in thermal imaging, indicating their relative low temperature compared with the surrounding area. After excluding the possibilities for other causes of localized temperature variances, the area most likely identifies a roof leak. This phenomena is apparent when viewing thermal imaging acquired in the early morning when ambient temperatures are rising. Conversely, localized areas of relatively warmer temperatures often indicate roof leaks during periods of cooling, such as those that occur near dusk.

If multiple images in both the thermographic spectrum and the visual spectrum are taken and are visually compared and contrasted, it is possible for trained technicians to identify roof leaks with high accuracy in lowslope roofs.

Figure 1 is a representation of a typical low-slope roof that has a leak. In the pre-dawn timeline (left image), the entire roof section is the same temperature (with the exception of vents and like structures, represented by small circles at lower left in all images). At dusk, the area of the roof leak is apparent (center image), as it is relatively warmer than the surrounding areas. In the early morning timeframe (right image), the leak area also is easily identified, as it is cooler than the surrounding area (conversely to what is found at dusk). 
Figure 1. Relative temperatures of low-slope roofs as viewed through a thermal camera.

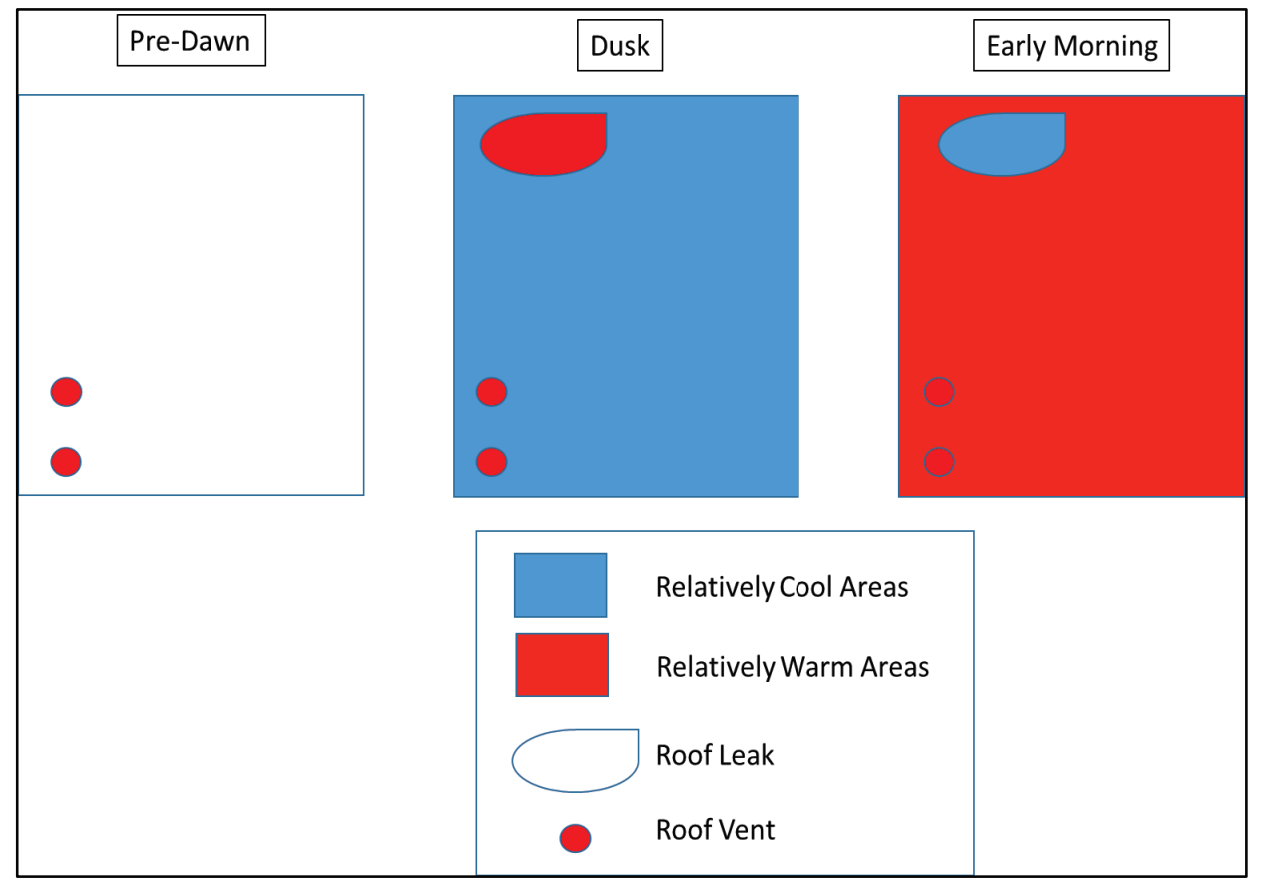

The science of leak detection via thermal imaging depends only on relative temperature variances; actual temperatures are not required. As such, it is possible that leaks can be detected when solar reflectance is present; however, false positives may be encountered. Therefore, thermal imaging is best done when solar reflectance is minimal-near or shortly after dusk and shortly after dawn. Furthermore, to reduce thermal reflectivity, it is best to image normal to the rooftop (i.e., 90 degrees to the nearly flat roof).

\subsection{Traditional thermal roof imaging acquisition}

Traditional thermographic roof imaging for identifying roof leaks has largely been conducted by roof inspectors who physically traverse roofs with handheld thermal cameras. Although proven effective, this method of image acquisition requires the acceptance of safety risks by the individuals who gather the data, most often in periods of low visibility (night). The resolution of the handheld imagery is high due to the close proximity of the camera; however, it is difficult to photograph the whole roof section in one photo. Moreover, the efficiency of handheld data acquisition is comparatively low when compared with alternative methods. 
Another method of thermal roof image acquisition is by using conventional aircraft. Although not used to the extent of handheld imagery, imagery captured from fixed-wing aircraft and helicopters has been used with success to identify roof leaks. The shortcomings of this approach include image resolution as the aircraft must operate at comparatively high altitudes when compared with handheld acquisition. Additionally, the scheduling of flights is problematic because weather conditions greatly affect the ability of the aircraft to fly and the efficacy of imagery to detect leaks. In addition, many roof managers have found the costs associated with conventional aircraft imagery acquisition are prohibitive, especially if the portfolio of roofs is rather small.

\subsection{Unmanned aircraft system thermal imaging}

Through the advent and maturation of UASs, the use of the aerial platform for thermal imagery collection has recently become a viable option and offers new benefits. The UAS platform can hover at the optimum altitude to capture thermal images. The platform is rapidly deployable and represents a potential cost-effective acquisition option due to its data collection efficiency, relatively low operating costs, and low purchase cost.

There are inherent challenges, however, that are associated with the use of UASs by the U.S. Army. UAS flights in national airspace require the acquisition of a Certificate of Authority (COA) to operate a UAS, issued by the Federal Aviation Administration (FAA), and an Airworthiness Release (AWR) from the U. S. Army Aviation and Missile Research, Development, and Engineering Center's (AMRDEC) AED for both the location of planned flights and the aircraft to be used. Coordination of radio frequencies also is required, as well as filing a Notice to Airmen (NOTAM) with the FAA that advises pilots in the area that UAS flights will be conducted.

Other challenges include those associated with physical flight and imagery acquisition. Although operation of UAS technology has become much more intuitive in recent years, practice is still required to operate UASs safely. Training also is required in the procedure for gathering quality imagery, which includes the optimal altitude of flights, the use of first person view (FPV; aircraft is always within line-of-sight), and other pertinent considerations. 


\section{Equipment for UAS Thermal Imaging}

Assessments of the thermal camera-equipped UAS started with the assembly and customization of the UAS at the ERDC-CERL location in Champaign, Illinois. The UAS procured by ERDC-CERL, although available as a COTS system, was not assembled. After significant assembly time, the visual spectrum and thermal payloads were added by using COTS gimbals. ${ }^{2}$ Table 1 displays the equipment used to create ERDC-CERL's UAS thermal system.

Table 1. UAS equipment list.

\begin{tabular}{|l|l|}
\hline DJI S1000+ & Unmanned aircraft system \\
\hline Flir Tau 2 336 & Thermal camera, 9 mm lens \\
\hline Horizon Hobby GB200 & Gimbal, modified for thermal camera \\
\hline GoPro Hero 4 Black & $\begin{array}{l}\text { Visual-spectrum camera and videorecorder } \\
\text { with wide-angle lens }\end{array}$ \\
\hline DJI Zenmuse H4-3D & Gimbal for GoPro Hero 4 Black \\
\hline Everfocus EMV200S & Digital video recorder for thermal video feed \\
\hline Futaba T14SB & Radio controller \\
\hline Fatshark Attitude V2 & FPV goggles \\
\hline Raspberry Pi & Onboard computer \\
\hline Teensy ++ 2.0 & Onboard computer \\
\hline
\end{tabular}

At the time of procurement, the Flir Tau 2 thermal camera did not have an accompanying gimbal. Subsequently, the Horizon Hobby GB200 was altered for use as a gimbal by the thermal camera. 3

The Flir camera was controlled by the Raspberry Pi, Arduino (Teensy++ 2.0), and a second Futaba radio receiver-all mounted onboard the aircraft. The Raspberry Pi General Purpose Input/Output (GPIO) interrupts were too slow to detect the pulse width from the pulse position modulation (PPM) signal received by the radio. Therefore, the Arduino-compatible

\footnotetext{
$2 \mathrm{~A}$ gimbal is a pivoted support that allows rotation of an object in multiple axes.

${ }^{3} \mathrm{~A}$ gimbal is now commercially available for an appropriately sized Flir thermal camera.
} 
Teensy++ 2.0 was necessary to decode the PPM signal and relay commands to the Raspberry Pi (see Appendix A for code). The Raspberry Pi converts the relayed commands to a serial data packet that is sent to the Flir camera via a universal serial bus (USB; see Appendix B). These command data packets for the Flir were not available in the documentation, so they were computed and assembled manually by the team (see Appendix C). The Flir camera is equipped with enough storage to store 100 digital thermal images, each with an image resolution of $336 \times 224$ pixels. Additionally, an EverFocus digital video recorder (DVR) equipped with global positioning system (GPS) capability was used to capture analog thermal video via the composite video port on the Flir. This video was used for further analysis and acquisition of additional analog frame stills from the mission. 


\section{Assessments at ERDC-CERL}

Early training flights of the UAS apparatus were in vacant, grassy areas of the ERDC-CERL compound. Significant training time was invested to ensure that operators were skilled in piloting the aircraft. After flight training, ERDC-CERL researchers conducted flights over the Central Plant mechanical facility (Figure 2).

Figure 2. Location of Central Plant Facility on ERDC-CERL's campus.

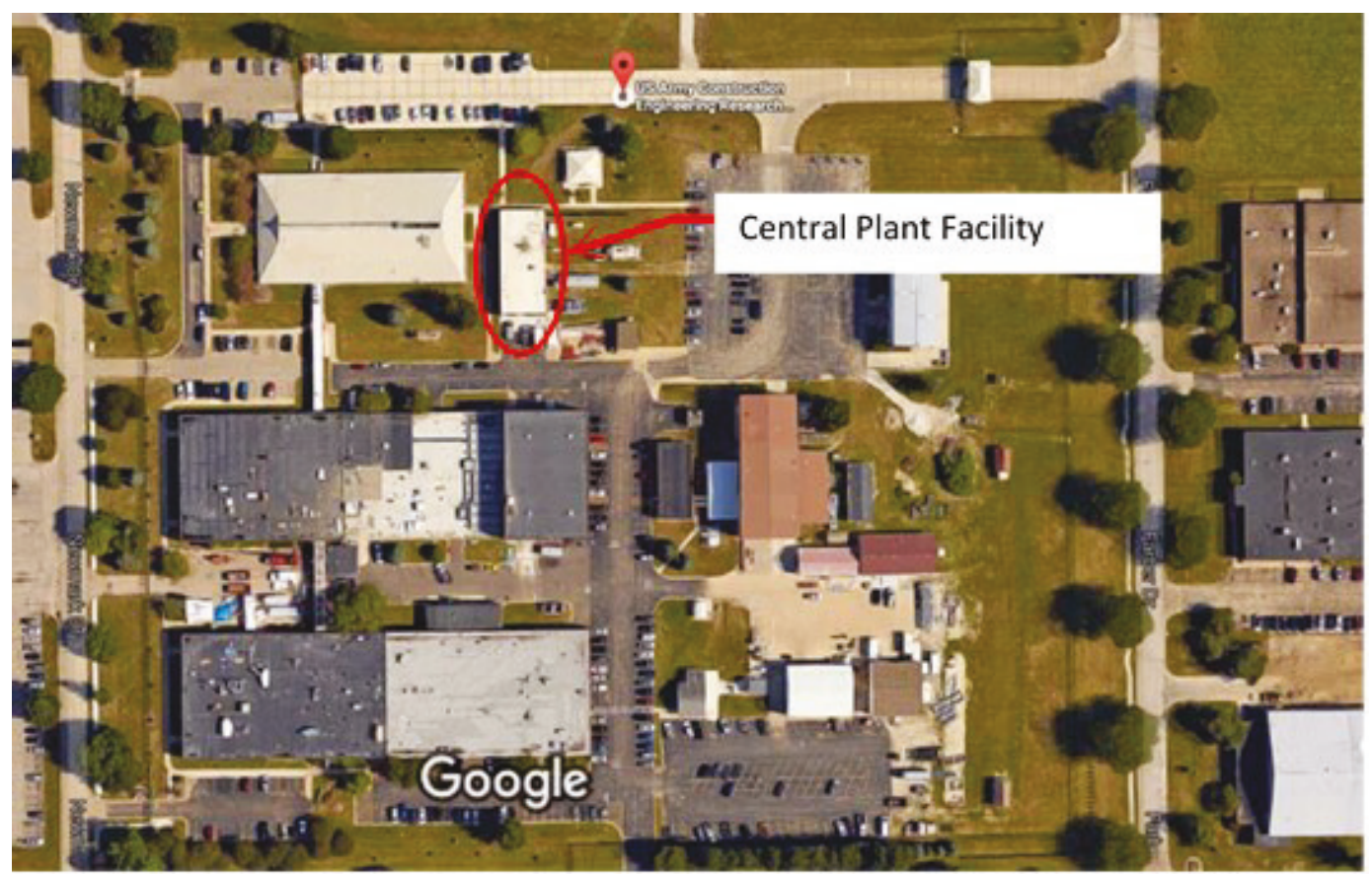

The subject roof consisted of a single-ply plastic membrane. After multiple thermal images were acquired through multiple flights, two areas consistently appeared darker in the images, indicating relatively cool areas. Because the roof membrane was rather new, the team questioned the data. After interviewing the building manager, however, the team found that there were indeed leaks in the subject roof. (Researchers had no knowledge of roof leaks before conducting flights.)

FPV was used to ensure the aircraft was positioned correctly over the intended roof section. FPV was not used to navigate, and the UAS was never out of the pilot's line-of-sight. 
The UAS thermal imaging revealed two leaks. The leak identified in the upper right corner of Figure 3 indicated a previous roof leak that supposedly had been repaired by the contractor under roof warranty. The leak identified to the lower left was an active roof leak, only recently identified by the building manager. The University of Illinois Urbana-Champaign (building owner) subsequently used these images to submit a claim on the contractor's repair under roof warranty.

Based on the results from the ERDC-CERL location testing, the team concluded that a UAS is capable of delivering thermal imaging of sufficient resolution to detect roof leaks.

Figure 3. Visual spectrum and thermal images of ERDC-CERL's Central Plant roof.
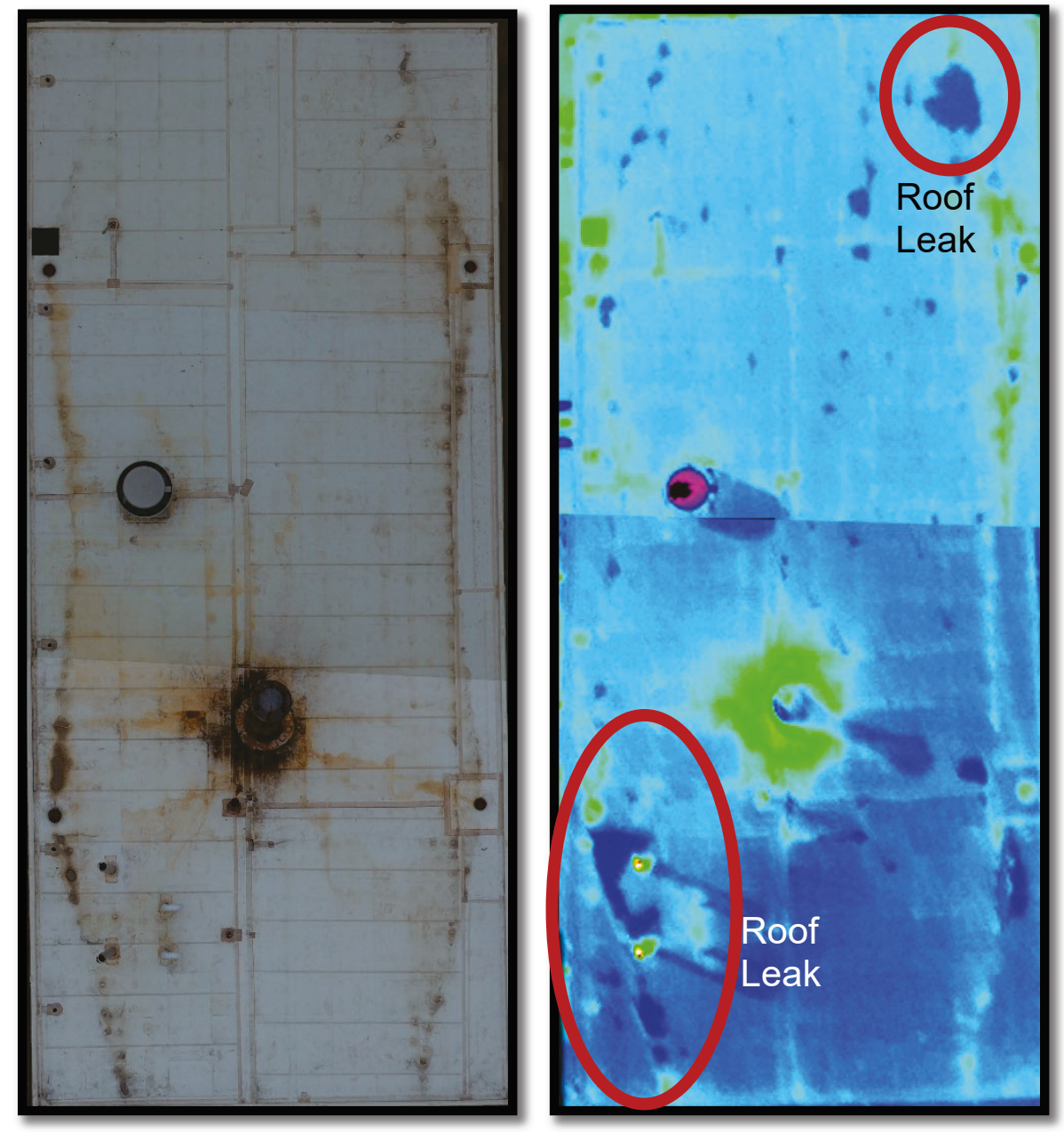


\section{Assessments at Fort Leonard Wood}

Roof assessments were also conducted on two facilities located at the Fort Leonard Wood training quarry. The training quarry area is located in restricted airspace that is controlled by the Fort Leonard Wood Range Control. Flights were coordinated and permission to conduct flights was granted by Fort Leonard Wood Range Control.

Two roofs were observed, as pictured in Figure 4. Both roofs visually appeared to be single-ply EPDM (ethylene propylene diene monomer) membrane on a pitched roof

Figure 4. Red circles indicate locations of two building rooftops assessed at Fort Leonard Wood quarry.

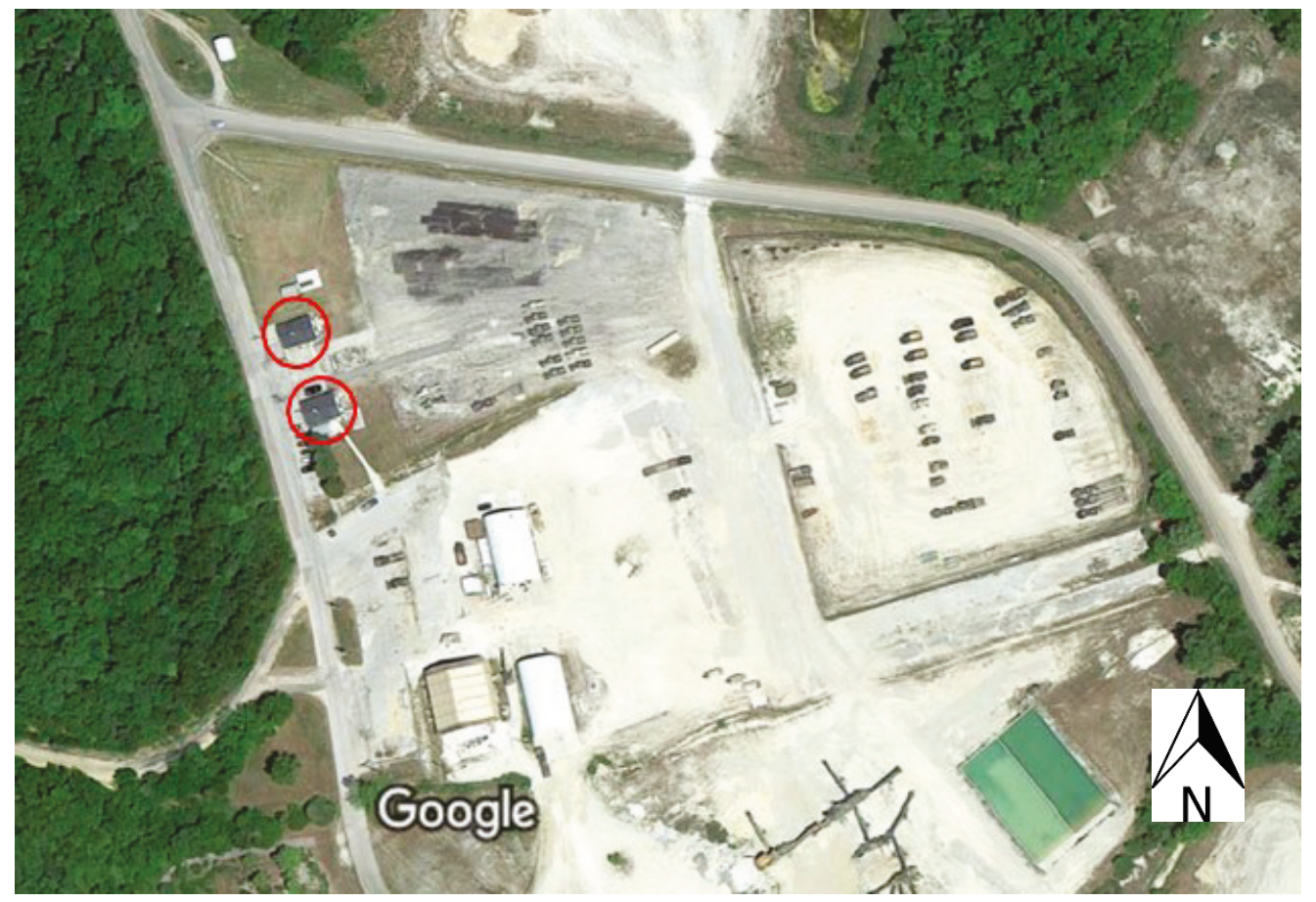


A thermal image of the northernmost of the two buildings (left side, Figure 5 and Figure 6) shows an area which appears darker near the center exhaust fan, indicating a relatively cool area. In the photograph at the right side of Figure 5, a light area coincides with the same area identified in the thermal image. Upon review of all photos, thermal images, and $4 \mathrm{k}$ video, the team determined that the EPDM membrane had been removed in the identified area. The identified area was reported to the roofing point of contact at the Fort Leonard Wood Directorate of Public Works (DPW).

Figure 5. Northernmost quarry building's roof images (thermal image, left side; photograph, right side), with UAV positioned directly over roof).

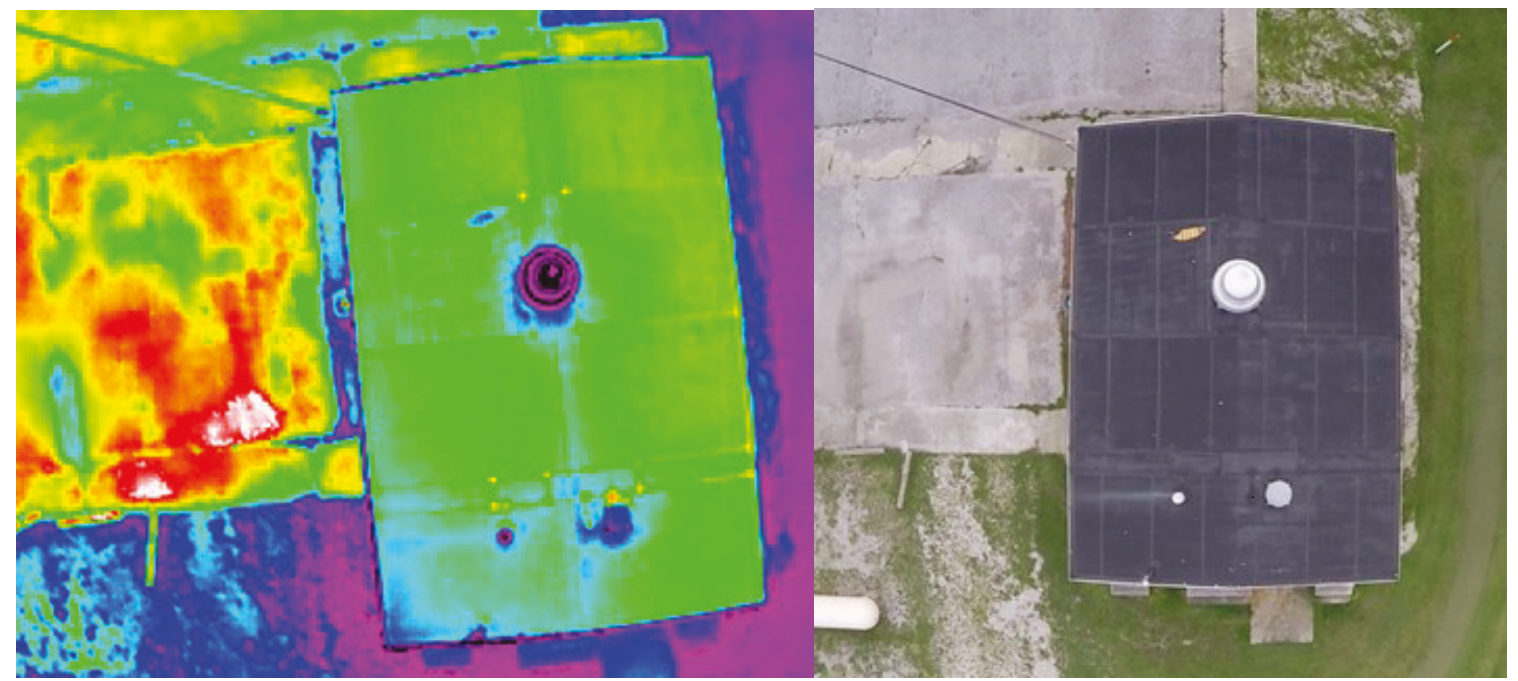

Figure 6. Northernmost quarry building, thermal image roof (UAV positioned to the south of the facility).

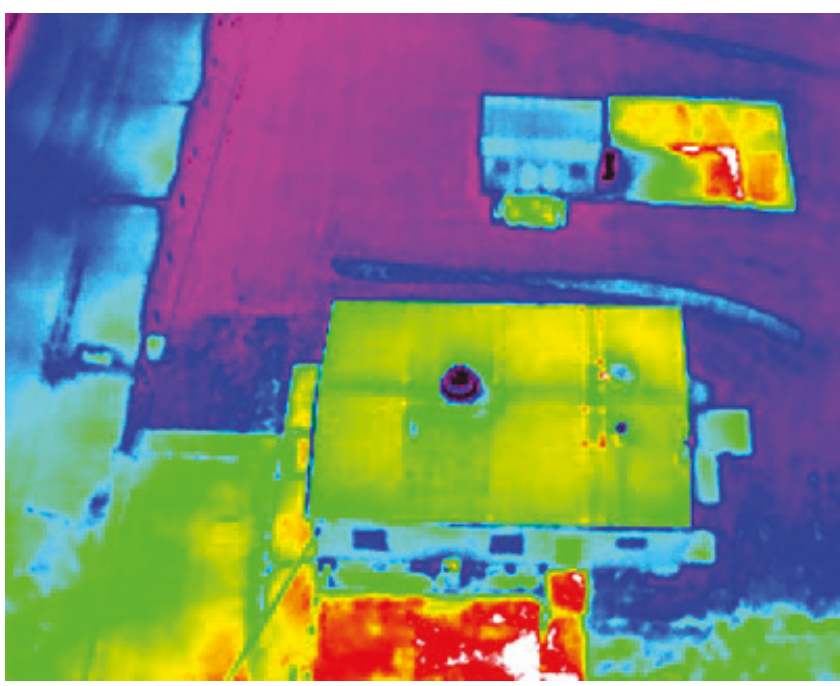




\section{Comparison of Thermal Roof Leak Detection Methods and Feasibility for Use by Department of Army}

\subsection{Efficacy}

Handheld thermal imaging by roof inspectors/technicians is effective in detecting roof leaks because of high image resolution. However, because technicians haven't a means to gather data from a significant altitude at a normal angle to the roof plane, the images are likely to show significant solar radiation and thus must be taken at nighttime to gather optimal results.

Traditional manned aircraft, both fixed-wing and helicopter, can perform aerial inspections. Because of the altitude at which a fixed-wing aircraft (and to a lesser extent, helicopters) must operate, the data resolution is compromised when compared to competing inspection methods. Small leaks and thermal bridges are very difficult to distinguish when using traditional manned aircraft. Additionally, weather conditions affect traditional manned aircraft much more so than competing inspection methods. These conclusions were reached in part by interviewing Mr. Jeffrey "Lake" Lattimore, an ERDC-CERL researcher who performed thermal roof leak inspections from a helicopter in fiscal year 2015 at Fort Leonard Wood.

Image resolution with a UAS is optimal because it can take both largescale photos, taken from higher altitudes, along with very close images. As with all forms of thermal image acquisition, thermal radiation is a concern. However, because a UAS can easily hover normal to the roof, a UAS can operate during morning and evening daylight hours-hours without the interference of solar radiance. (Solar radiance is not reflected toward the UAS during the morning and evening hours.)

Figure 7-Figure 9 illustrate the resolution of the most similar-sized facility (Building 750) assessed in the helicopter study to that of one obtained by the UAS of a much smaller quarry facility. As can be seen, the image resolution of the much smaller quarry facility is much higher than that obtained by helicopter of the larger Building 750. The quarry facility's roof area is approximately 1,000 sq. $\mathrm{ft}$., while Building 750's roof area is approximately $7,000 \mathrm{sq} . \mathrm{ft}$. 
Figure 7. Visual spectrum image of Building 750 (lower left), acquired by helicopter during the 2015 ERDC-CERL project.

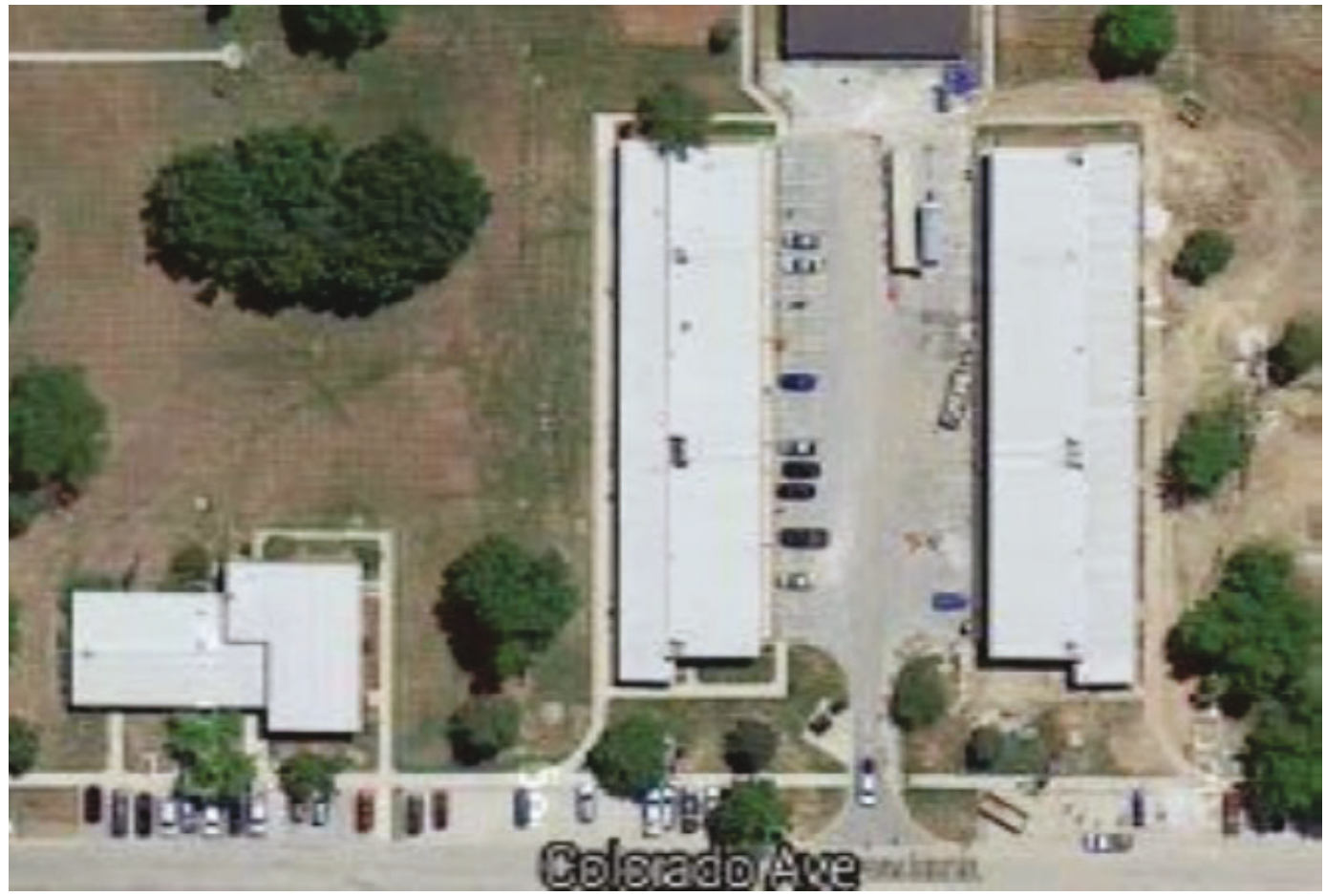

Figure 8. Thermal spectrum image of Building 750 (lower left), acquired by helicopter during the 2015 ERDC-CERL project.

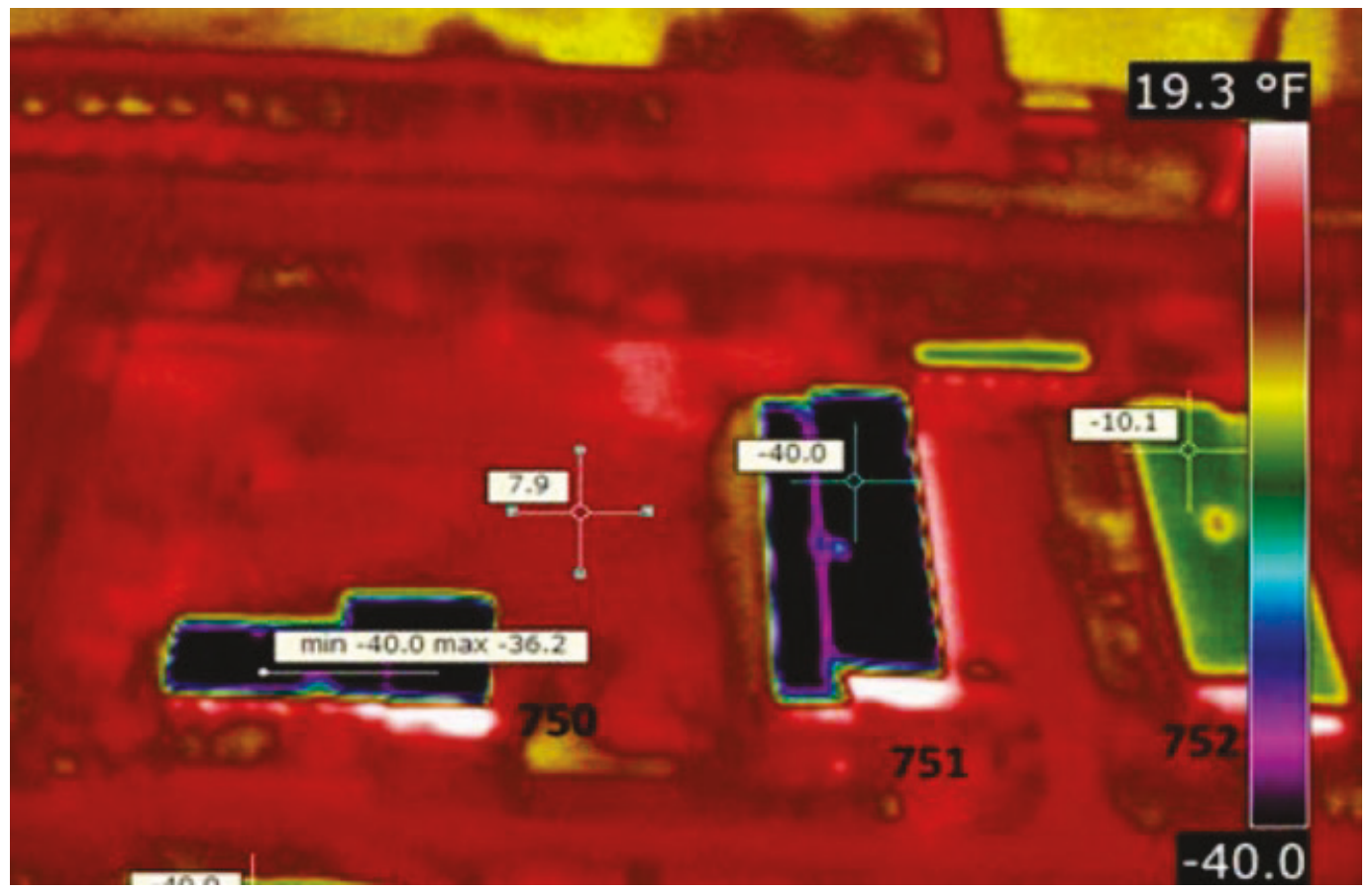


Figure 9. Thermal image of quarry facility (right side) obtained by UAS.

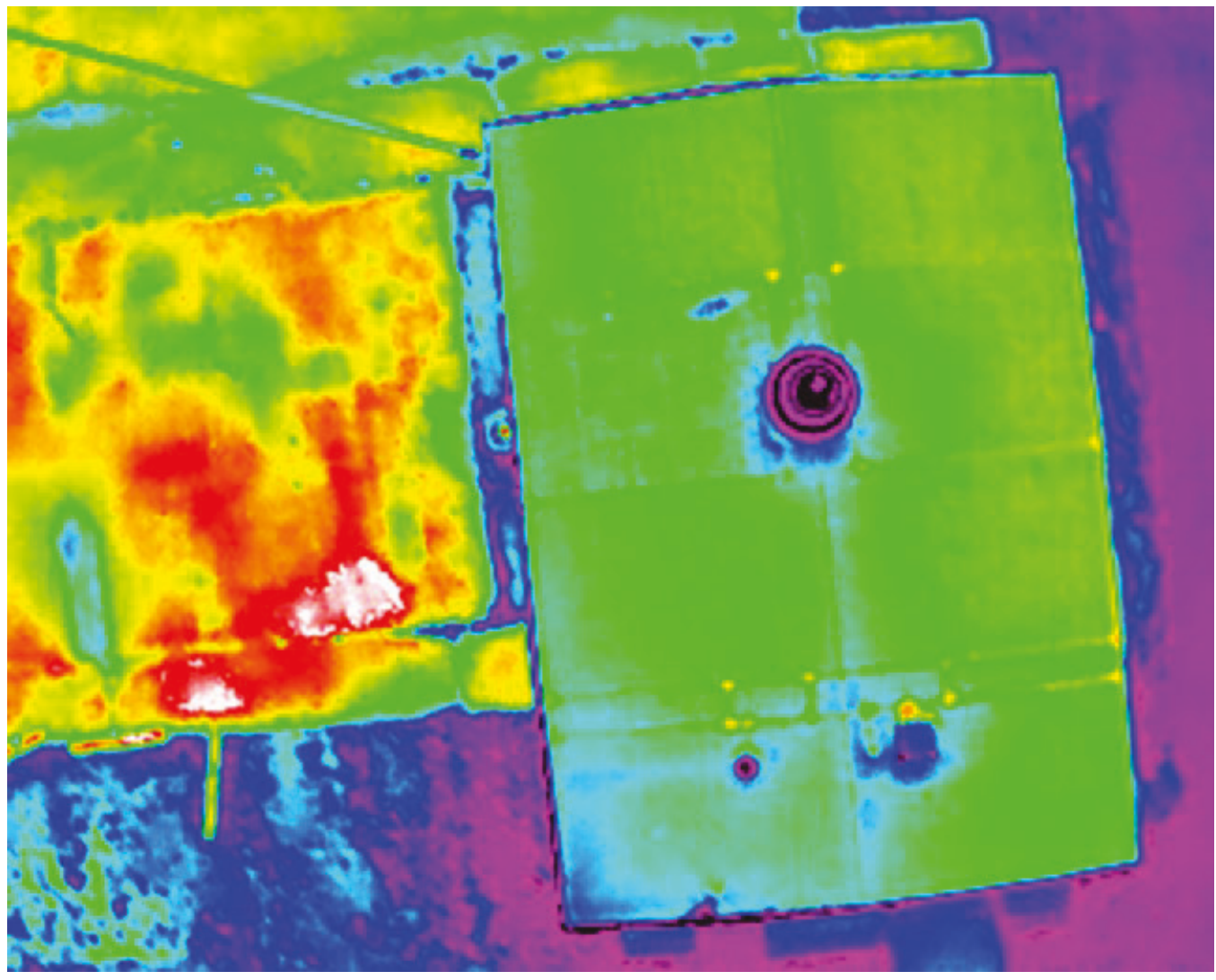

Although not part of this study, full roof inspections are appropriate to be performed by the UAS platform because of its use of a combination of the thermal and visual spectrum cameras, FPV capability, and the ability to hover at varied altitudes. This potential for full roof inspections is discussed in Chapter 8, "Recommendations for Future Research."

\subsection{Efficiency}

Handheld, rooftop thermal imaging is relatively inefficient if the purpose of such thermography is merely to obtain thermal images. The costs and time required of a roof inspector to gain access to a facility rooftop and the inability to take images of multiple roofs simultaneously renders this method quite inefficient. Because handheld thermal leak detection is ineffective during the daytime, any prospective efficiencies gained by conducting traditional full-roof inspections (such as those required for use of the ROOFER program) are moot. However, there is little preparation required of users to use handheld thermal imagers. 
Roof thermal imaging by manned fixed-wing aircraft or helicopter is very efficient in regards to time of acquisition. Multiple roofs are assessed simultaneously with this method-potentially a whole installation's cantonment area can be imaged in a single flight, depending on the size of the installation. The only downside in terms of time efficiency is the possibility that weather conditions could curtail a scheduled flight, extending the time required to receive images and likely increasing costs. Another consideration is the availability of aircraft and crew, which may result in long lead times compared with alternatives.

UAS roof thermal imaging is equivalent in efficiency to manned fixed-wing aircraft or helicopter and is far more efficient in terms of time of acquisition when compared with handheld thermal imaging. As UASs can operate, pursuant to the FAA/Department of the Army memorandum of agreement (MOA), at higher elevations than current regulations allow commercial companies or hobbyists, it is feasible that UASs can duplicate the results seen by manned aircraft.

The time required to create the necessary documents and attain required signatures for UAS must be considered in regard to efficiency. The process's duration is variable, and one cannot estimate the time required to produce and attain signatures, as well as the time the AED and FAA take to issue applicable approvals. However, after gaining approvals to operate the UAS, such approvals remain in place for two years. If done in coordination with other UAS uses, the overhead costs expended on flight approval can be dispersed.

\subsection{Safety}

In terms of safety, handheld thermal image acquisition is relatively dangerous to the inspector due to the potential to fall from a rooftop. When conducted nocturnally, handheld inspections are particularly dangerous.

Manned aerial thermal imaging acquisition is relatively safe to operators, bystanders, and property. Manned aircraft have undergone stringent testing and pilots are highly trained.

UAS thermal imaging acquisition safety is a complex issue. There is higher probability a UAS will fail in flight than will conventional manned aircraft. 
In over 40 hours of CERL testing, no safety incidents occurred which jeopardized the well-being of bystanders or property. However, this is not a sufficient duration of testing to determine the reliability of the UAS. However, the likely consequence of UAS failure, relative to handheld and manned aircraft failure, is low.

Figure 10 and Figure 11 are graphical representations of ERDC-CERL's estimation of a frequency-consequence matrix. The metric for frequency is as discussed previously in this section. The metric for the determination of consequence is the loss of life and/or property in failure.

Figure 10. Frequency/consequence matrix.

\begin{tabular}{|c|c|c|c|c|c|}
\cline { 2 - 5 } \multicolumn{1}{c|}{} & Insignificant & Minor & Moderate & Major & Severe \\
\hline $\begin{array}{c}\text { Almost } \\
\text { Certain }\end{array}$ & Medium & Medium & High & High & Extreme \\
\hline Likely & Medium & Medium & Medium & High & Extreme \\
\hline Possible & Low & Mediun & Medium & High & High \\
\hline Unlikely & Low & Low & Medium & Medium & High \\
\hline Rare & Low & Low & Low & Medium & High \\
\hline
\end{tabular}


Figure 11. Frequency of incident vs. consequence of incident occurrence.

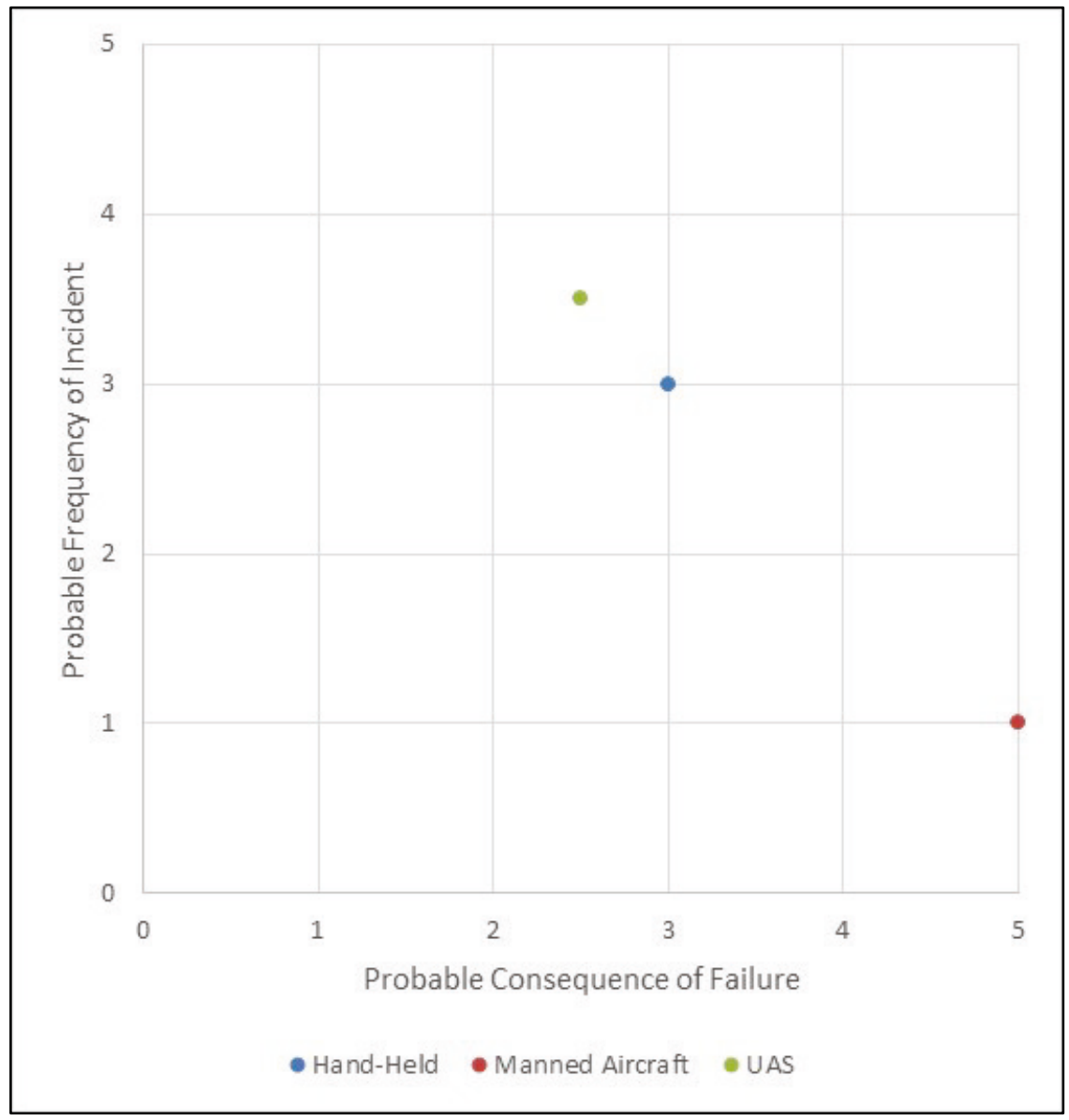

\subsection{Cost comparison}

For the purposes of programming and feasibility analysis, Table 2 displays a cost estimate and comparison of techniques produced with the knowledge ascertained in this study.

Table 2. Cost estimate and comparison of thermal roof-leak detection techniques.

\begin{tabular}{|c|c|c|c|c|c|c|c|c|}
\hline & $\begin{array}{c}\text { Cost of } \\
\text { Equipment }\end{array}$ & $\begin{array}{c}\text { Useful life of } \\
\begin{array}{c}\text { Equipment } \\
\text { (years) }\end{array}\end{array}$ & $\begin{array}{c}\text { Services per } \\
\text { Year (full } \\
\text { capacity) }\end{array}$ & $\begin{array}{c}\text { Average Labor } \\
\text { per Service } \\
\text { (hours*) }\end{array}$ & Labor Rate & $\begin{array}{l}\text { Roofs per } \\
\text { Service }{ }^{* *}\end{array}$ & $\begin{array}{c}\text { Cost per } \\
\text { Roof }\end{array}$ & $\begin{array}{c}\text { Cost per } \\
\text { Square Foot** }\end{array}$ \\
\hline Hand-Held & $\$ 3,500.00$ & 10 & 1320 & 1 & $\$ \quad 100.00$ & 1 & 100.27 & $\$ 0.01337$ \\
\hline Manned Aircraft* ${ }^{*}$ & NA & NA & & $7,500.00$ & NA & 100 & 75.00 & $\$ 0.01000$ \\
\hline UAS (as tested) & $\$ 20,000.00$ & 5 & 2640 & 0.5 & $\$ \quad 100.00$ & 3 & 17.17 & $\$ 0.00229$ \\
\hline \multicolumn{9}{|c|}{${ }^{*}$ Total manned flight costs shown in Labor per Service } \\
\hline${ }^{* *}$ Based upon an & erage roof si & ize of 7500 sq. & & & & & & \\
\hline
\end{tabular}

As shown in Table 2, the estimated costs for UAS thermal roof-leak detection are significantly lower than the alternatives. UAS-delivered thermal imaging saves approximately $83 \%$ when compared with the handheld 
technique, and $77 \%$ of the manned aircraft alternative. (The manned aircraft alternative does not include procurement of equipment, assuming the service will be contracted out.)

Another alternative is to use Army aircraft on training missions to gather thermal data as the costs per flight are reduced dramatically by a multipurposed flight. This option was not considered, as the results from the 2015 ERDC-CERL research from a helicopter rendered images with resolutions insufficient to detect roof leaks. Availability of such aircraft at all installations is also a concern with this approach.

The estimate does not consider the overhead necessary to acquire flight approvals because of the variability of effort needed by different user organizations. This expense is significant in the first year, but the expense is significantly lessened in subsequent years. If the Army were to implement UAS thermal roof-leak detection in a centralized manner (see Chapter 7 , "Outline of Implementation Plan for Army Installations"), the costs for the acquisition of flight approvals could more easily be determined and would likely be less costly than the sum of costs of individual components gaining independent flight approvals.

\subsection{Conclusion}

When considering the relatively high efficacy, efficiency, safety attributes, and costs, the UAS thermal roof-leak detection option is the best choice from an engineering perspective. It is the most effective way to identify roof leaks because of its ability to hover at low or high altitudes. It, along with manned aircraft, are the most efficient means of gathering thermal imagery. Although the safety attributes relative to alternatives are debatable, UASs perform well when considering the product of the probability of failure and consequence of failure. Finally, UASs are simply less expensive than other alternatives.

The analysis did not include the public's view of UAS, as that was not the objective of the study. However, the public's view often considers a UAS to be the equivalent of a "spy drone," and such a view could have an impact on the probability of implementation. 


\section{Outline of Implementation Plan for Army Installations}

\subsection{An Army UAS roof-leak detection team}

Proactive management of roof leaks is possible through thermal imaging techniques. Given the sizable consequences of roof leaks and the scale of the Army's low-slope roof portfolio of 225 million square feet, contemplation of a centrally managed UAS thermal imaging team is a worthwhile exercise. The complexity of UAS flight approvals, operation of the UAS, and roof construction and inspection familiarity add to the necessity of developing a centrally managed team.

The team would travel between installations and be comprised of three roof experts, able and willing to be trained on UAS operation, as well as being supported in administrative functions through an organization such as a U.S. Army Corps of Engineers (USACE) District.

Although the return on investment (ROI) cannot be calculated because the average cost of a roof leak in the Army does not exist, the total yearly costs for a UAS thermal roof-leak detection team have been estimated by this study (Table 3). Handheld and manned aircraft alternatives are included only to compare relative cost; based on this study, the best choice for Army roof-leak detection is UAS, delivered as stated in section 6.5, "Conclusion."

Unlike the estimate created in section 6.4 ("Cost comparison"), Table 3 (next page) includes an estimate of flight-approval costs. This added estimate is possible because a centralized method for obtaining those approvals has been assumed.

Other assumptions used in the Table 3 estimate are listed below:

- "Initial Training" includes development of training materials as well as in-depth initial training by ERDC-CERL and experienced pilots.

- "Initial Flight Approvals" includes the development and signature of the SOP and System Safety Management Plan (discussed in Chapter 9). 
Table 3. Estimated cost of Army UAS thermal roof-leak detection team.

\begin{tabular}{|c|c|c|c|c|c|c|c|c|c|}
\hline & $\begin{array}{c}\text { Cost per } \\
\text { Square Foot }\end{array}$ & $\begin{array}{c}\text { Initial } \\
\text { Training }\end{array}$ & $\begin{array}{l}\text { Annual } \\
\text { Training }\end{array}$ & $\begin{array}{l}\text { Initial Flight } \\
\text { Approvals }\end{array}$ & $\begin{array}{c}\text { Annual Flight } \\
\text { Approvals }\end{array}$ & Annual Travel & $\begin{array}{c}\text { Year } 1 \\
\text { Implementation }\end{array}$ & $\begin{array}{l}\text { Subsequent } \\
\text { Yearly Cost }\end{array}$ & 3 Year Cost \\
\hline Hand-Held & $\$ 0.01337$ & $\$ 50,000.00$ & $\$ 5,000.00$ & $\$$ & $\$$ & $\$$ & $\$ 1,052,651.52$ & $\$ 1,007,651.52$ & $\$ 3,067,954.55$ \\
\hline \begin{tabular}{|l} 
Manned \\
Aircraft \\
\end{tabular} & $\$ 0.01000$ & $\$$ & $\$$ & $\$ 200,000.00$ & $\$ 100,000.00$ & $\$ 40,000.00$ & $990,000.00$ & $890,000.00$ & $\$ 2,770,000.00$ \\
\hline $\begin{array}{l}\text { UAS (as } \\
\text { tested) }\end{array}$ & $\$ 0.00229$ & $\$ 100,000.00$ & $\$ 15,000.00$ & $\$ 500,000.00$ & $\$ 125,000.00$ & $\$ 100,000.00$ & $871,717.17$ & $411,717.17$ & $\$ 1,695,151.52$ \\
\hline
\end{tabular}

*Each year $1 / 3$ of the portfolio is inspected 
Note that the UAS thermal roof-leak detection team concept would be difficult to staff with contracted employees. The flight approval process currently dictates that a GS-15, O-6 equivalent (or above) accept the risks associated with such flights. Clearly, a problem arises when the government would be accepting risks on the behalf of contractors for their conduct.

\subsection{Discussion}

If one hypothetically assumes that the cost of an average roof leak is in the range of $\$ 5,000-\$ 500,000$, with an average value of $\$ 25,000$, then the number of roof leaks which would need be detected (that weren't evident to the occupant by observed leakage) to offset the yearly cost of just over $\$ 400,000$ is approximately 16 leaks. ERDC-CERL researchers are reasonably confident that such a team would find more than 16 previously unidentified roof leaks within a yearly inspection area of 75 million square feet, given that such a roof leak was detected on only 5,000 square feet of inspected roof in this study. This hypothetical is obviously inexact, but reasonable.

It stands to reason that if manned roof inspections (as required for roof management systems such as ROOFER) could be replaced by UAS inspections, then the economics of the hypothetical discussed in the above paragraph would become even more advantageous to the government.

If other data could be collected during roof inspections by the UAS team, the economic model would change advantageously. Some other data collection opportunities for such a team include:

- aerial 2D map making;

- plant health monitoring;

- water tower, electrical structure, and radio tower inspection;

- facility envelope inspection: visual and thermal; and

- pipe location and leak detection. 


\section{Recommendations for Future Research}

\subsection{Evaluation of UAS as replacement}

The evaluation of a thermally equipped UAS as a replacement for traditional manned rooftop inspections is an important next step to this study. One could estimate that such an inspection will not be as accurate or as thorough as the product of a highly experienced roof inspector. However, given the high costs of experienced roof inspectors (considering their low relative efficiency), the potential UAS replacement study would be a worthwhile endeavor.

\subsection{Discovery of other UAS applications}

Another suggestion for future research is discovering other applications for a similarly equipped UAS. (i.e., similarly equipped as required for roof inspections). Much like a Swiss Army knife, the UAS team's value grows with corresponding reductions in the number of other "tools" previously needed to perform tasks.

\subsection{Development of UAS toolkit}

UAS procurement, flight approvals, and technical knowledge (both application-specific and UAS-specific) are all difficult to acquire in the Department of Defense (DoD) at the present time. The development and maintenance of a UAS toolkit, complete with a means of procurement (General Services Administration [GSA] catalog or similar), streamlined flight approvals, technical knowledge that includes support of the hardware and software, and application-specific knowledge would make the benefits of UASs much more likely to be realized. 


\title{
9 Unmanned Aircraft System Flight Approval Process
}

As of this document's date, UAS flights in the Army are not governed by FAA rules and procedures that apply to the commercial sector or the hobbyist sector-that is, the former Section 333 (FAA 2012) or the newer Part 107 (FAA 2016). Instead, the Department of the Army operates in the national airspace pursuant to a formal memorandum of agreement (MOA) between the Department of the Army and the FAA. The MOA covers all types of flights, including small UAS (i.e., one that weighs less than 20 pounds). 4

Although multiple forms and documents will be created in the pursuit of flight approval for small UAS (as detailed below), two documents are required to operate a UAS-a COA issued by the FAA and an AWR issued by the AMRDEC AED.

It is best to first contact the AED before contemplating UAS operations. The AED is intimately familiar with rules and regulations, and it also is upto-date with seemingly constantly changing flight procedures. The AED may be contacted at the following:

\author{
U. S. Army Aviation and Missile Research, Development, and \\ Engineering Center (AMRDEC) \\ Aviation Engineering Directorate \\ Unmanned Aircraft Systems Division \\ Bldg. 5400, Redstone Arsenal, AL 35898 \\ (256) $313-6456$
}

Because the AED is a fee-for-service organization, funds must be transferred to AED for an AWR to be released. The AWR is a required document for both the location(s) of contemplated flights and the aircraft. Aircraft for which there hasn't previously been an AWR issued may be re-

\footnotetext{
${ }^{4}$ A larger UAS would follow a different set of rules.
} 
quired to be reviewed by AED headquarters physically at Redstone Arsenal, Alabama. Due to this fact and as was just stated, it is suggested that prospective users contact the AED before procuring a UAS.

The four documents required to be completed in order to gain a COA and AWR are listed below.

1. Unmanned Aircraft System Standing Operating Procedure (SOP)

2. System Safety Management Plan

3. UAS model-specific Material Risk Acceptance Letter

4. UAS model-specific Operational Risk Acceptance Letter

The SOP and the System Safety Management Plan documents are completed within the using organization, and multiple internal signatures are required. The SOP and System Safety Management Plan require periodic review and constant updates. The two risk acceptance letters (Material Risk Acceptance Letter and Operational Risk Acceptance Letter) need to be signed by an O-6 (GS-15 equivalent) or above.

The overall goal during creation of these documents is to consider all possible hazards to people, property, and other air traffic, and to take steps to mitigate or lessen those risks. A well-developed SOP and System Safety Management Plan, along with the knowledge gained from the development of those documents, will better ensure the safe operation of the UAS and the comfort level of those signing the documents.

\subsection{Standing operating procedure development}

Flight approval starts with creating the SOP of the organization requesting approval to operate the UAS. (A template SOP is included in Appendix D.) The SOP establishes the responsible individuals in the UAS flight program and their respective responsibilities, and it delineates the rules and procedures required of the organization's UAS program as well.

Because the document is complex and requires multiple signatures, it is likely that its development will take substantial resources and will require multiple iterations. 


\subsection{System Safety Management Plan development}

The System Safety Management Plan includes much of the data created in the SOP, but it expounds on the safety aspect of UAS flights and includes greater detail on the specific remedies for incidences. (A template is included in Appendix E.)

\subsection{Operational Risk Acceptance Letter}

The Operational Risk Acceptance Letter, as with the Material Risk Acceptance Letter, is straightforward to create from the template provided in Appendix F. Like the Material Risk Acceptance Letter, the essence of the risks accepted through signature of this letter relate to lack of MIL-STD810G (DoD 2008) test data. The letter requires O-6 (GS-15) or higher signature, and the letter is UAS model-specific.

\subsection{Material Risk Acceptance Letter (UAS model-specific)}

The Material Risk Acceptance Letter is straightforward to create from the template provided in Appendix G. The DoD conducts lengthy, detailed, and expensive tests of aircraft to quantify their airworthiness and risks associated with flight. Since the UAS hasn't undergone extensive airworthiness qualification because such tests are cost prohibitive, the aircraft is considered unreliable. The material risks which are being accepted by the signatory are those risks associated with this lack of test data. The letter requires signature by an O-6 (GS-15) or higher.

\subsection{Restricted airspace}

A COA is not required to conduct UAS flights in DoD restricted airspace. Since the space (at the time the MOA is active) is not part of the national airspace and is controlled solely by the DoD, no COA is required. However, the restricted airspace must be outside a five nautical mile-radius created by a controlled airport. Since most areas of interest at Army installations are within either the national airspace or within the five nautical mile-radius around a controlled airspace, the practical uses of UASs without a COA are few. 


\section{References}

DoD. 31 October 2008. MIL-STD-810G "Environmental Engineering Considerations and Laboratory Tests." Washington, DC: Department of Defense, Defense Standardization Program.

FAA. 2012. Section 333, "Special Rules for Certain Unmanned Aircraft Systems." Under Subtitle B (Unmanned Aircraft Systems), FAA Modernization and Reform Act of 2012. Washington, DC: Federal Aviation Administration.

. 29 August 2016. Part 107, "Small Unmanned Aircraft Systems." Under Title 14 (Aeronautics and Space), Chapter 1 (Federal Aviation Administration, Department of Transportation) - Volume 2, Subchapter F (Air Traffic and General Operating Rules). Washington, DC: Federal Aviation Administration. 


\section{Appendix A: Arduino Source Code}

Appendix A includes the source code (as modified by ERDC-CERL researchers from example code for the Arduino or Teensy microcontroller) to communicate with the Futaba Radio Control (RC) receiver. The Raspberry $\mathrm{Pi}$ is unable to monitor the rise and fall of pulses at the speed the $\mathrm{RC}$ receiver is sending the pulses. The time between the pulses is related to the position of the switches on the RC transmitter. This modified code interprets the position of the switches and relays the command to the Raspberry Pi GPIO pins. 


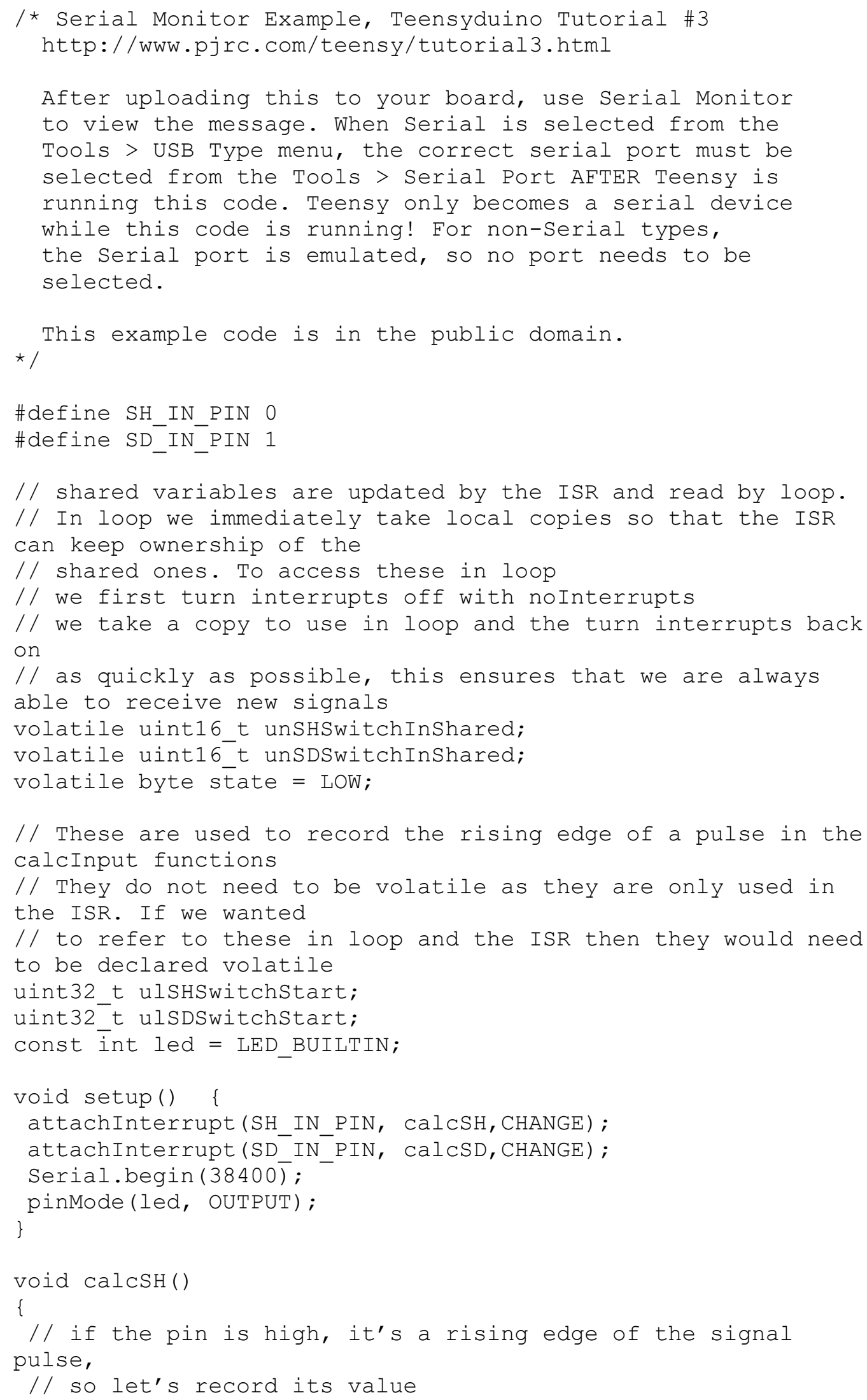




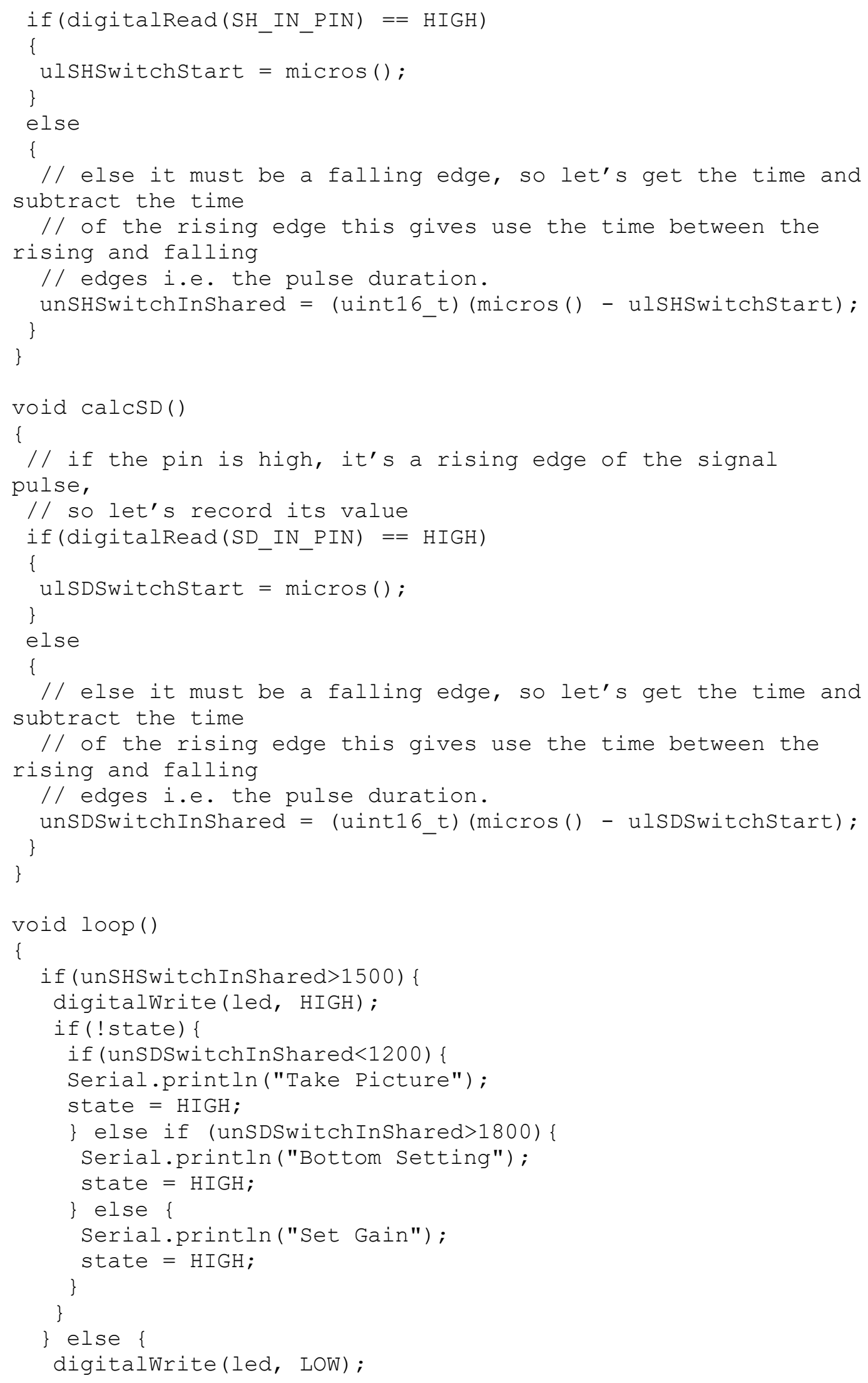


state = LOW;

\}

\} 


\section{Appendix B: Raspberry Pi, Flir Camera Control Source Code}

Appendix B contains the source code compiled in C for the Raspberry Pi which is required to communicate between the Arduino and the Flir Camera. The Flir camera is attached to one of the Raspberry Pi universal serial bus (USB) ports (/dev/ttyUSBo). The Arduino is attached to the GPIO ports, and the code listens for the GPIO ports to rise. When a rise is detected, this code interprets the command and sends the command to the Flir Camera. 


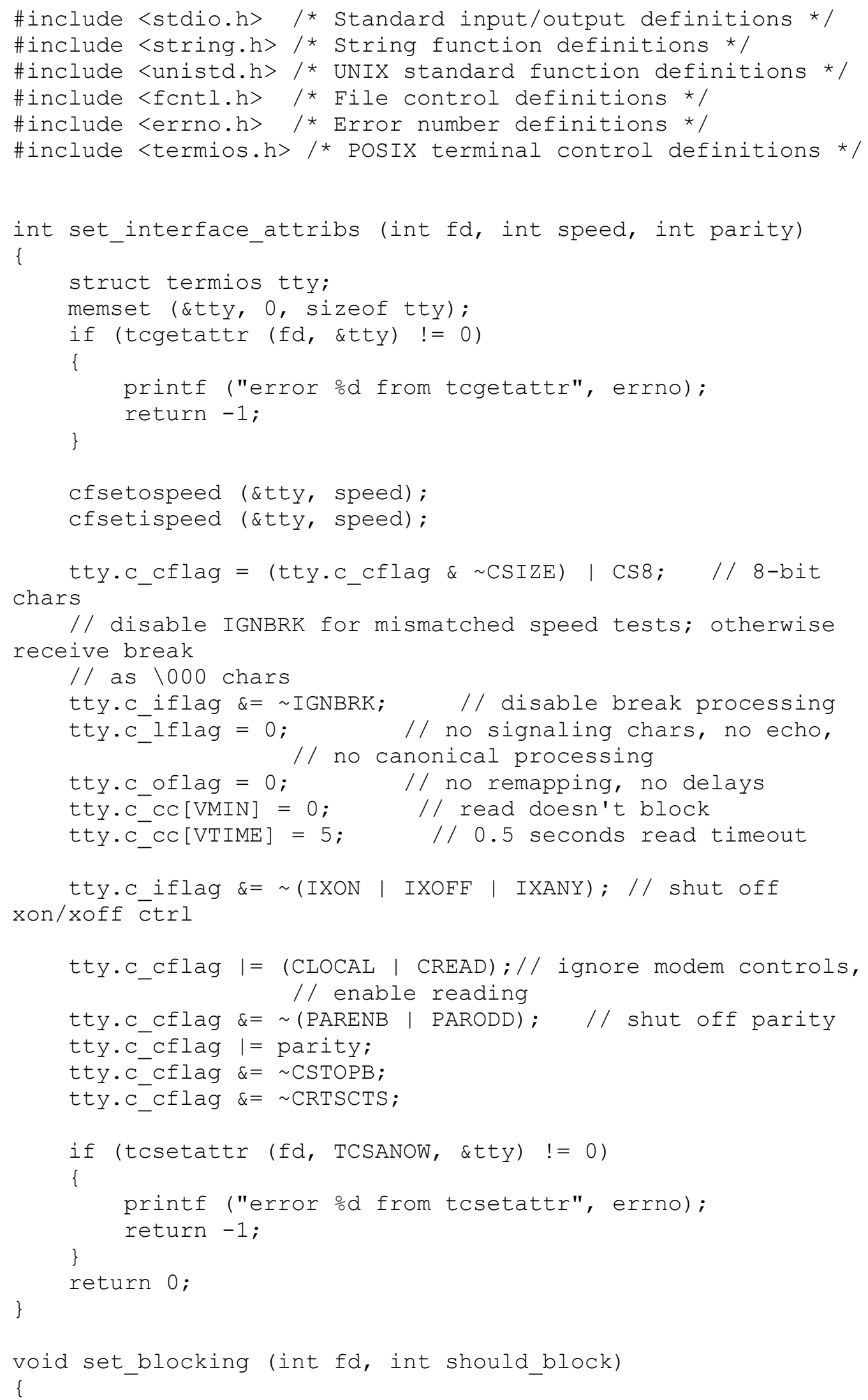




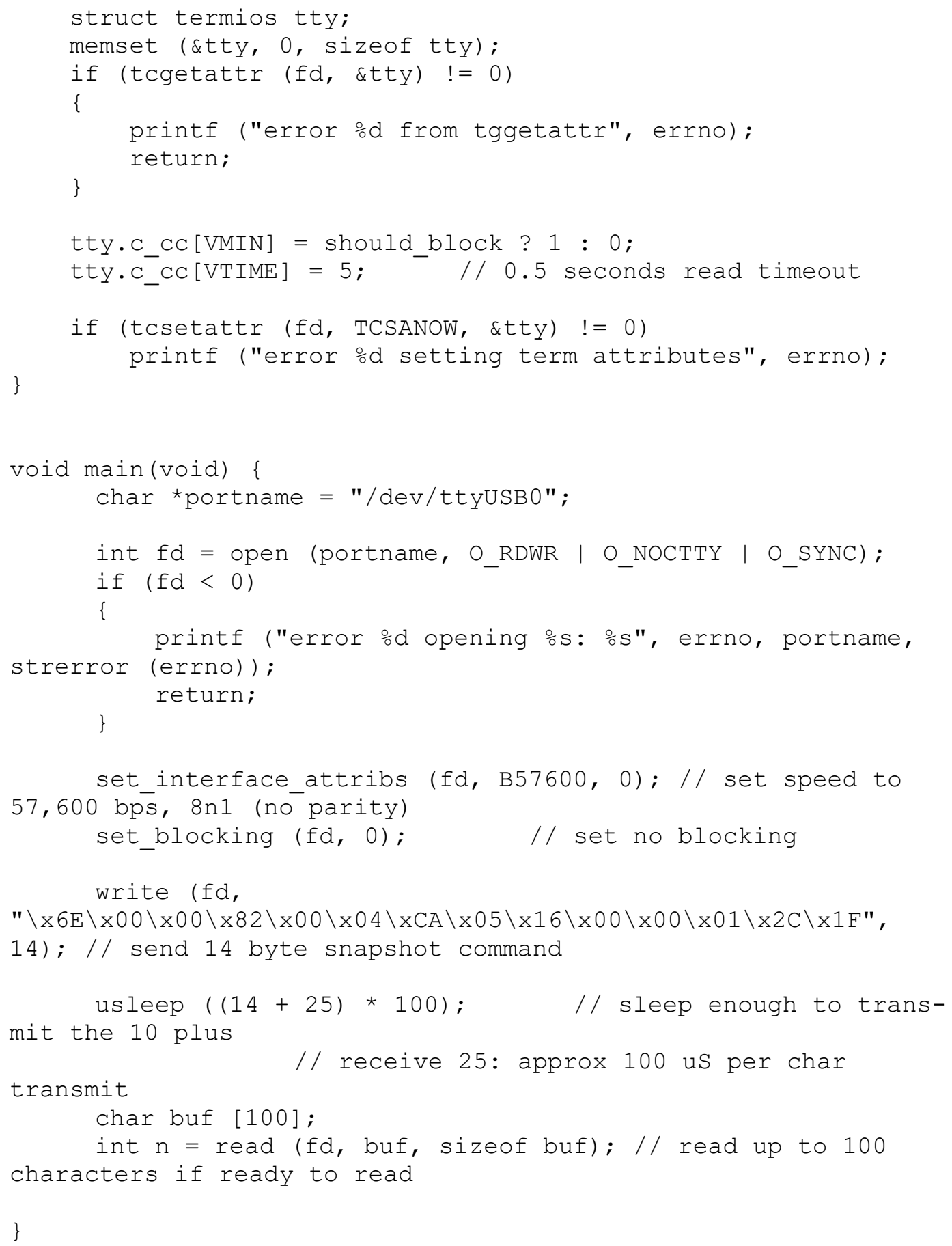




\section{Appendix C: Flir Thermal Camera Commands}

Appendix C contains a list of the commands as used by the source code in Appendix B to control the Flir camera. 
AGC Manual:

6E;00;00;13;00;02;E5;CA;00;03;30;63

AGC Auto Bright:

6E;00;00;13;00;02;E5;CA;00;02;20;42

AGC Plateau Histogram (Default):

6E;00;00;13;00;02;E5;CA;00;00;00;00

Reboot

6E;00;00;02;00;00;B1;DB;00;00

Meter Display Off

6E;00;00;2B;00;02;89;CE;00;00;00;00

Meter Display Numeric Only

6E;00;00;2B;00;02;89;CE;00;01;10;21

Meter Display Thermometer Only

6E;00;00;2B;00;02;89;CE;00;02;20;42

Meter Display Thermometer and Numeric

6E;00;00;2B;00;02;89;CE;00;03;30;63

Take Snapshot to Next File Position

$$
\text { 6E;00;00;82;00;04;CA;05;16;00;00;01;2C;1F }
$$

Take Snapshot to File Position 1

$$
\text { 6E;00;00;82;00;04;CA;05;16;01;00;01;1B;2F }
$$

Get Number of Snapshots

$$
\text { 6E;00;00;D6;00;04;48;0B;FF;FE;00;13;91;A2 }
$$

Display Snapshot at File Position 1 


$$
\text { 6E;00;00;82;00;04;CA;05;17;01;00;01;6D;9B }
$$

Display Snapshot at File Position 2

$$
\text { 6E;00;00;82;00;04;CA;05;17;02;00;01;34;CB }
$$

Resume Video

$$
\text { 6E;00;00;82;00;04;CA;05;17;00;00;00;4A;8A }
$$

Set Video Palette \#1 (White Hot):

$$
\text { 6E;00;00;10;00;02;BC;9A;00;00;00;00 }
$$

Set Video Palette \#2 (Black Hot):

6E;00;00;10;00;02;BC;9A;00;01;10;21

Set Video Palette \#3 (Color 1):

6E;00;00;10;00;02;BC;9A;00;02;20;42

Set Video Palette \#4 (Color 2):

$$
\text { 6E;00;00;10;00;02;BC;9A;00;03;30;63 }
$$




\section{Appendix D: Standing Operating Procedure Template/Sample for Unmanned Aircraft System Army Flight Approval}

The following sample is reproduced here to inform other users of what is needed to create their own SOP and UAS Army flight-approval documents. 


\section{Organization Name \\ Sub-Organization Name}

\section{STANDING OPERATING \\ PROCEDURE}

FOR

\section{Operation of Unmanned Aircraft Systems (UAS)}

SOP Number: 1000-1-1

Revision Number: $\mathbf{n} / \mathbf{a}$

Change Number: $\mathbf{n} / \mathbf{a}$

Date: 23 September 2016

\section{Residual Risk Assessment Code (RAC): LOW}

MEDIUM

This Standing Operating Procedure is in compliance with Army Regulation 385-10 (Safety), The Army Safety Program, and Department of the Army Pamphlet 385-30 (Safety), Mishap Risk Management, and Occupational Safety and Health Administration (OSHA) Standards and Requirements. These regulatory publications mandate a program of hazards analysis and risk assessment, personnel qualifications and training, written operating procedures, and risk mitigation using prudent safe work practices, engineering controls, and personal protective clothing and equipment. 


\section{INTERNAL REVIEW / RISK ACCEPTANCE PAGE:}

1. STANDING OPERATING PROCEDURE FOR: Unmanned Aircraft Systems

2. OPERATION / PROCESS / TASK TYPE: Flight Operations of UAS

3. SOP NO.: 1000-1-1

DATE:23 Sept. 2016

4. REVISION NO.: $\mathrm{n} / \mathrm{a}$

DATE: $\mathrm{n} / \mathrm{a}$

5. CHANGE NO.: $\mathrm{n} / \mathrm{a}$

DATE: $\mathrm{n} / \mathrm{a}$

6. PREPARING DIRECTORATE/FUNCTION/ OFC SYMBOL:

7. RESIDUAL RISK ASSESSMENT CODE (RAC):

8. PREPARED BY:

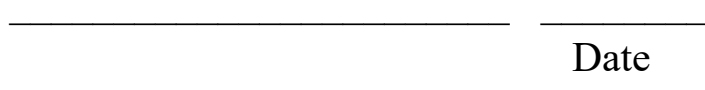

9. TECHNICAL REVIEW:
OFC SYMBOL:

TITLE:

(Tel \#):
OFC SYMBOL:

TITLE:

(Tel \#):

10. SAFETY REVIEW:

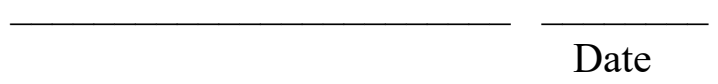

OFC SYMBOL:

TITLE:

(Tel \#): 
11. CONCURRENCE BY MANAGEMENT / DATE:

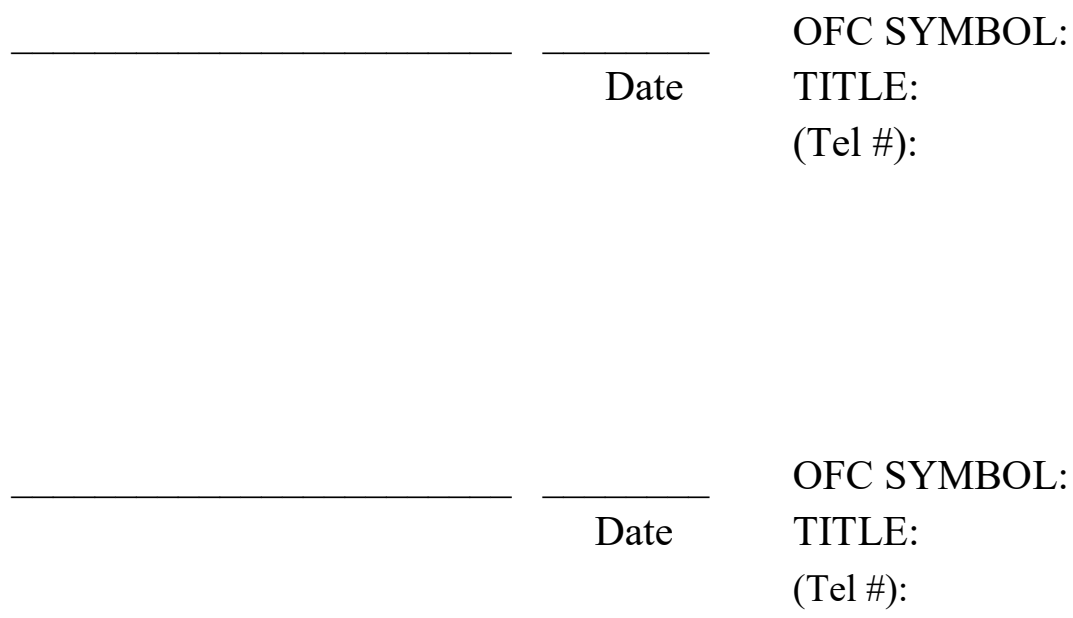

12. FINAL APPROVAL (RISK ACCEPTANCE - LOW):

OFC SYMBOL:

Date TITLE: (O-6, GS-15 or above) (Tel \#): 


\section{ANNUAL REVIEWS*}

I have reviewed this SOP and assessed its accuracy and applicability for the specific operations and determined that there are no changes to the facilities, operations, materials, tooling, or other parameters that increase safety risk or invalidate the original risk assessment.

\begin{tabular}{lll}
\hline & & OFC SYMBOL: \\
Date & TITLE: \\
& $($ Tel \#):
\end{tabular}

$\begin{array}{lll} & & \text { OFC SYMBOL: } \\ \text { Date } & \text { TITLE: } \\ & (\text { Tel \#): }\end{array}$

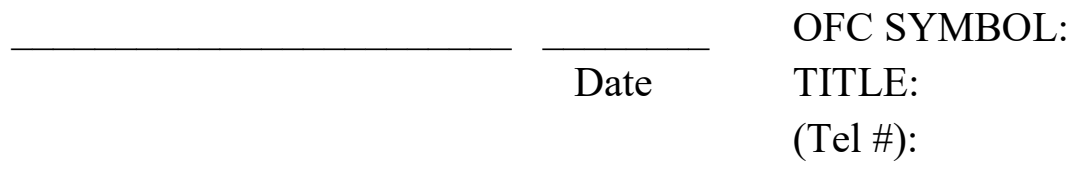

*Annual reviews are required by AMCR 700-107/AR 385-10. Any change to this SOP before its Annual review requires re-staffing. 


\section{SUPERVISOR AND “RESPONSIBLE INDIVIDUAL(S)" STATEMENT}

THE SUPERVISOR \& PERSONNEL ASSIGNED AS "RESPONSIBLE INDIVIDUAL(S)" WHO DIRECT OR AFFECT THE ACTIONS OF OTHERS WILL:

- MAINTAIN A SAFE AND HEALTHFUL WORK ENVIRONMENT.

- INSPECT WORK AREAS FOR POTENTIAL HAZARDS PRIOR TO INITIAL START OF OPERATIONS AND PERIODICALLY THEREAFTER.

- PROMPTLY TAKE ACTION AS REQUIRED TO CORRECT THE HAZARDS.

- ENSURE A JOB HAZARD ANALYSIS IS CONDUCTED AS PART OF PLANNING, DEVELOPMENT AND PREPARATION OF ALL OPERATIONS AND INTEGRATE CORRECTIVE ACTIONS INTO THE SOP.

- ENSURE ALL PERSONNEL ARE RESPONSIBLE FOR ACCIDENT PREVENTION.

- ENSURE ALL GOVERNMENT AND CONTRACTOR PERSONNEL ARE PROPERLY TRAINED TO PERFORM THE OPERATIONS AND WORK TASKS DELINEATED IN THIS SOP.

- $\quad$ TAKE THE NECESSARY ACTION(S) TO ENSURE THAT PERSONNEL FOLLOW PRUDENT, SAFE WORK PRACTICES, SAFETY GUIDELINES, STANDARDS, AND REGULATIONS, INCLUDING WEARING REQUIRED PERSONAL PROTECTIVE EQUIPMENT (PPE).

- $\quad$ ENSURE THAT THE NECESSARY PROCEDURES FOR ACCIDENT/INCIDENT NOTIFICATION AND EMERGENCY RESPONSE ARE DOCUMENTED AND UNDERSTOOD.

- BE RESPONSIBLE FOR AND BE HELD ACCOUNTABLE FOR ACCIDENTS/INCIDENTS, PROPERTY DAMAGE AND ENVIRONMENTAL CONTAMINATION AS A RESULT OF SAFETY VIOLATIONS OCCURRING DURING OPERATIONS UNDER THEIR DIRECT SUPERVISION AND CONTROL. 
1. The Supervisor and individual(s) with assigned responsibility/authority (referred to as "Responsible Individual(s)") for occupational safety and environmental compliance will read and sign this statement.

2. I have personally reviewed the procedures and hazard control measures delineated in this SOP and confirm that the operation can be performed in a safe, efficient, and environmentally compliant manner. I have verified that all Government and/or contractor personnel conducting the operation are qualified, have been properly trained and are capable of performing in a safe manner. I have verified that each employee has read this SOP, understands the safety precautionary measures and understands that these procedures must be followed without deviation (unless prior approval is obtained in writing from the Responsible Individual(s)).

3. I acknowledge that anyone involved with the operation prescribed in this SOP may call a halt to any activity if they perceive that it has or will become an immediate danger to safety, health and/or property.

4. The Supervisor/Responsible Individual(s) as the person(s)-in-charge will sign (below) when first assigned responsibility for conduct of an operation prescribed in this SOP, and:

a. Annually, acknowledging this Statement of Responsibility,

b. Prior to restarting an operation that is intermittent (non-routine) or has not been performed for 90-days or more,

c. When a change or modification is made to this SOP (procedure or equipment).

\begin{tabular}{|l|l|}
\hline $\begin{array}{l}\text { SUPERVISOR \& “RESPONSIBLE } \\
\text { INDIVIDUAL'S" SIGNATURE }\end{array}$ & \\
\hline & DATE \\
\hline & \\
\hline & \\
\hline & \\
\hline
\end{tabular}




\section{OPERATOR/TASK PERFORMER STATEMENT}

1. Employees assigned as an operator/task performer (Government or contractor) will read and sign this statement.

2. I have read and understand the general and specific requirements contained in this SOP pertaining to prudent and safe work practices, hazard control and environmental protection necessary to safely conduct the operation. Further, I will wear required personal protective equipment (PPE) identified in this SOP for my personal protection. I am knowledgeable of the potential hazards and level of risk associated with performing operations contained in this SOP. I will ensure that equipment and tools used are properly inspected and clean to facilitate their safe operation. I will report any unsafe work conditions or unsafe acts to my Supervisor/Responsible Individual(s). I agree to abide by these instructions for all operations I am involved with and for any procedure or activity defined herein.

3. I understand that I have the authority to stop any activity prescribed herein that I perceive has or will become an immediate danger to safety, health and/or property.

4. The operator/task performer will sign (below) when first assigned responsibility for performing an operation or work task prescribed in this SOP, and:

a. Annually, acknowledging this Statement of responsibility,

b. Prior to restarting an operation that is intermittent (non-routine) or has not been performed for 90-days or more,

c. When a change or modification is made to this SOP (procedure or equipment).

\begin{tabular}{|l|l|}
\hline OPERATOR/TASK PERFORMER SIGNATURE & DATE \\
\hline & \\
\hline & \\
\hline & \\
\hline & \\
\hline
\end{tabular}




\subsection{PURPOSE:}

This document describes the operational procedures and operator selection/training requirements necessary to ensure safe flight operations of the UAS by personnel within Organization Name, SubOrganization Name.

The Army is incorporating Small Unmanned Aircraft System(s) (SUAS) into the inventory. Currently there is not a clear description from the Army on how to handle development of SUAS equipment not part of a Program of Record. Many of these SUAS efforts either support the warfighter indirectly by use as a research asset or support domestic missions CONUS. Sub-Organization personnel have developed this SOP to guide the safe use of all SUAS air vehicles in the Sub-Organization inventory.

\subsection{DESCRIPTION OF OPERATION:}

This document describes UAS manual and autonomous flight operations, maintenance and safety considerations, and personnel requirements related to the operation of multirotor aircraft within the UAS family of configurations.

SUB-ORGANIZATION procured the research assets within the UAS family to serve as a research platform for sensor development and experimentation. The primary use of the platforms is aerial video coverage of controlled research events such as thermographic research and visual inspection research.

Note: This SOP must be present in the operational area of use. A copy will also be maintained in a file within the office of the technical lead or organizational manager.

\subsection{APPLICABILITY:}

This SOP is applicable to all government and contractor personnel who are actively involved in air or ground operations related to the UAS vehicles. Operational procedures described in this SOP will be used to maintain prudent, safe operating practices and to ensure that appropriate response actions are taken in the event of an emergency. 
Airworthiness oversight of the UAS assets is documented in a Level 3 Airworthiness Release (AWR) as issued by the Special Projects Team, Unmanned Aircraft Systems Division, and Aviation Engineering Directorate (AED) of RDECOM. This SOP is subordinate to the Level 3 AWR and the SUB-ORGANIZATION System Safety Management Plan. Additionally, this SOP shall be subordinate to the SOP's or regulations of National Airspace controlled by the FAA, Restricted Airspace, any relevant agreements between the DOD and FAA or research ranges where the UAS is operating.

\subsection{RESPONSIBILITIES:}

\subsection{General}

The roles and responsibilities of personnel involved in both the performance and in the occupational health and safety, explosives safety, and environmental protection oversight of the operations described in this document are defined in Section 2 of the SUB-ORGANIZATION System Safety Management Plan, Roles and responsibilities specific to the operation of the UAS.

\subsection{UAS Technical Lead}

A government employee within SUB-ORGANIZATION will be designated the UAS Technical Lead. This individual is responsible for the general configuration management and maintenance of the UAS fleet. The Primary Operator is responsible for managing all hardware and software configurations of the UAS fleet in accordance with the Army Airworthiness Release(s) issued by AMRDEC-AED. The UAS Technical Lead is also responsible for training and approving other SUB-ORGANIZATION personnel to serve as flight operators.

\subsection{Mission Commander}

A government employee within SUB-ORGANIZATION will be designated the Mission Commander (MC).The $\mathrm{MC}$ is responsible for planning, briefing and executing all flight operations. The $\mathrm{MC}$ is empowered to make decisions and to take the immediate corrective action necessary to prevent an accident, injury to third parties and/or flight crew personnel, and/or damage to Government and/or non-Government property or equipment. The $\mathrm{MC}$ is responsible for the safe conduct of the flight in any airspace and over any ground space for which operations are authorized by FAA COA, DoD-FAA MOA, on a training area, cantonment area or while operating in Active Restricted Airspace. The MC is the SUB- 
ORGANIZATION's representative to ensure that all established procedures, implied and specified, are followed, to the letter, in their spirit and intent. The MC must remain at the controlling site and providing over watch for the mission until the mission is completed or until properly relieved by another MC. The MC's duties include the following:

a. Assure that an assessment of the operational area meets any requirement prescribed for that location in accordance with FAA and/or Army guidance and policy.

b. All material hazards have been identified and eliminated or adequate mitigations have been implemented prior to flight operations.

c. A risk determination for the material hazards has been made and all risks assessed and accepted at the appropriate level in a signed risk acceptance memorandum to be provided to AED prior to flight operations.

d. All operational hazards have been identified and eliminated or adequate mitigations have been implemented prior to flight operations.

e. A risk determination for the operational area hazards has been made and all risks assessed and accepted at the appropriate level in a signed risk acceptance memorandum to be provided to AED prior to flight operations.

f. Supervision for all personnel involved in the mission.

g. Ensuring all personnel supporting the operation are qualified for the role they are assigned, have been properly briefed and thoroughly understand their role in the mission.

h. Logbooks are present and accurate prior to all flight operations.

i. $\quad$ All system equipment is verified in proper working order during the preflight inspection and properly set-up.

j. The mission data is recorded in the logbook.

k. Ensures that all required communications with the other controlling agencies are established and maintained throughout the entire operation.

I. A Mishap Action plan is in place prior to operations for each operational area with emergency notification procedures completed prior to flight. 
m. Conducts pre/post mission briefs with all personnel involved in the mission as prescribed within this SOP.

\section{$4.4 \quad$ Flight Operator}

A designated government employee UAS Flight Operator (FO) is responsible for the safe operation of the UAS aircraft under both manual and autonomous flight. For manual flight, the primary task of the operator is to start the unmanned aircraft (UA) and place it into GPS flight mode at/in the desired location at the operational altitude above training/test site. The operator will then place the vehicle into (autonomous hover or loiter or mapping grid mode) for the duration of operations or test. If applicable the UAS FO must remain ready to resume manual control from UAS autonomous flight mode under any condition. The operator is also responsible for complying with all local procedures outlined in this SOP, the specific area operational requirements as determined by the DOD FAA MOA or appropriate Aviation officer/Airspace manager/Range Control, pertinent FAA Certificates of Authorization (COAs) and Army Material Command (AMC) Waivers. The UAS FO will continuously advise the Safety Observer (SO) of the flight maneuvers he plans on conducting and request the Safety Observer clear the airspace before proceeding. In the event of any emergency the operator will if in the autonomous mode regain control the aircraft manually to the best of his/her ability and bring the aircraft to a safe landing or ditching zone that is briefed prior to take off. The FO has final decision on when to land or ditch the aircraft but may not violate FAA or Army regulations in an effort to "save" the UAS.

The same UAS FO is responsible for safe operation of the UAS under autonomous flight. During autonomous flight/hover, the primary task of the UAS operator is to monitor all system status indicators on the Ground Control Station (GCS). The UAS FO must remain ready to resume manual control of the UAS under any conditions. The operator will also maintain communication throughout the flight with the Safety Observer to ensure everyone knows what the other operators/observers are doing and who is controlling the UAS. 


\subsection{Safety Observer}

The UAS FO will always specify an SO and an Alternate Safety Observer (ASO) prior to flight. The SO is responsible for all communications with Air Traffic Control (ATC) and Range Control related to UAS operations. He/she is also responsible for keeping the FO aware of the need to change between manual and autonomous modes. The SO will monitor the authorized airspace to identify any errant aircraft or unsafe conditions that might exist during the flight. The SO must also monitor the surrounding area for personnel, ground and air vehicles, or other hazards natural and manmade (birds, wires, trees, etc.) and notify the UAS FO to avoid these areas. The SO must communicate with the UAS FO clearly and anticipate the movements of the aircraft to prevent an unsafe condition from developing into a mishap. In the event of an emergency, the SO will call the appropriate agency overseeing the airspace (ATC or range control, etc.) on the assigned radio frequency, or from a mobile or land telephone line. The SO will also continue to communicate with the FO on any potential environmental hazards that the operator has to be aware of while dealing with the emergency situation.

\subsection{Maintenance Personnel}

Maintenance will typically be performed by the UAS Technical Lead. Maintenance personnel are responsible for conducting all maintenance and upkeep required to ensure the safe operation of UAS. Personnel will authorize the use of each vehicle in the UAS fleet based on completion of appropriate inspection(s). Maintenance personnel, if distinct from the $\mathrm{FO}$, will assist in setting up the UAS for flight and conducting the preflight inspection as necessary. Communications related to safety will be conducted with the SO and with the lead research engineer. In the event of an emergency, the maintenance personnel will stand by for instructions from the FO in recovery of the air vehicle. 


\subsection{PERSONNEL QUALIFICATIONS:}

\subsection{Flight Operator}

The UAS FO will be approved for flight by the Mission Commander. Approval will follow the applicant passing full flight and hover testing on a simulator.

Each UAS FO will complete a minimum of four (4) flight hours annually. This requirement may be accomplished through flight simulation software and/or flights conducted with the UAS.

The UAS FO Trainee will conduct all initial qualification training using the flight simulation instruction. The UAS FO Trainee must demonstrate proficiency with the aircraft in all modes (e.g. GPS attitude mode turned off) prior to being recommended for solo flight.

The UAS FO Trainee must receive no less the four (4) hours of solo time and demonstrate proficiency during an evaluation for all the maneuvers listed below.

The UAS FO will complete a minimum of four flight operational missions annually. Each mission must include full configuration of the UAS (and its payload actuation if applicable) followed by a successful flight that demonstrates full mission functionality (e.g., captures overhead video of a test site).

\subsection{Safety Observer}

Any member of the UAS team who has read and understood all requirements and stipulations set forth in the UAS documentation package (SOP, AWR, FAA COA or FAA/DoD MOA requirements) meets the requirements necessary to act as an $S O$.

\subsection{Maintenance Personnel}

UAS team members other than the UAS Technical Lead may perform incidental maintenance and upkeep as long as they are qualified for the maintenance action, the Technical Lead is notified and the maintenance is documented. 


\subsection{QUALIFICATION TRAINING:}

6.1. As part of the flight training, all FOs will receive training in the following areas:

6.1.1. UAS systems including aircraft and transmitter.

6.1.2. Emergency procedures including radio interference and lost link procedures.

6.1.3. Crew coordination with an safety observer.

6.1.4. Vehicle starting, stopping, and servicing procedures.

6.1.5. Local operating procedures.

6.2. FOs will demonstrate ability to conform to common standards for required maneuvers as listed below.

6.2.1. For flight/hover maneuvers.

6.2.1.1. Control/drift less than $\pm 5 \mathrm{ft}$. longitudinally and laterally.

6.2.1.2. Maintain altitude $\pm 5 \mathrm{ft}$.

6.2.2. For forward flight maneuvers.

6.2.2.1. Maintain ground track during takeoff and landing.

6.2.2.2. Maintain altitude $\pm 10 \mathrm{ft}$.

6.2.2.3. Terminate landings to a flight/hover $\pm 10 \mathrm{ft}$.

6.2.2.4. Terminate landings to specified termination point, $\pm 20 \mathrm{ft}$. longitudinally and laterally.

6.3. FOs will conduct the training below annually to maintain qualification.

6.3.1. Review and discuss any applicable AWRs, MOAs, COAs.

6.3.2. Review and discuss this SOP.

6.3.3. Review and discuss system documentation/manuals.

6.3.4. Review and discuss emergency procedures. 


\subsection{LOCATION OF OPERATION:}

The UAS system will operate primarily in the designated training and research area. However, this SOP allows the UAS fleet of vehicles to operate in any airspace covered by the FAA/DoD Memorandum of Agreement for Operation of Unmanned Aircraft Systems in the National Airspace System.

\subsection{HAZARDOUS MATERIALS / AMMUNITIONS \& EXPLOSIVES}

No hazardous materials will be used as part of the operation of the UAS fleet/family of vehicles. However this SOP allows the UAS fleet/family of vehicles to operate in proximity to hazardous materials such as, but not limited to, ammunition and explosives (A\&E) on active test ranges. Safety concerns related to these hazardous materials items will be documented and proper safety procedures implemented.

\subsection{PERSONAL PROTECTIVE EQUIPMENT}

No specific personal protective equipment (PPE) is required to operate and maintain the UAS. This SOP does require all personnel to use appropriate PPE as defined herein or by the SOP of the test range where the UAS fleet/family of vehicles are operating.

10.0 REFERENCES:

\begin{tabular}{|l|l|}
\hline \multicolumn{2}{|c|}{$\begin{array}{c}\text { Reference Documents } \\
\text { (Applicable to Creation of this SOP) }\end{array}$} \\
\hline OSD 14887-07 & $\begin{array}{l}\text { MOA for Operation of UAS in the National Airspace } \\
\text { System } \\
\text { The Army Safety Program }\end{array}$ \\
\hline AR 385-10 & Army Safety Program \\
\hline DA PAM 385-10 & US Army Material Command (AMC) Safety Program \\
\hline AMCR 385-10 & AMRDEC Safety Management Plan \\
\hline AMRDEC 385-1-1 & System Safety Management Plan \\
\hline $\begin{array}{l}\text { SUB-ORGANIZATION- } \\
\text { SYS-SAFETY-MGT-PLAN }\end{array}$ & \\
\hline AR 95-23 & Unmanned Aircraft System Flight Regulations \\
\hline AR 385-95 & Army Aviation Accident Prevention \\
\hline FORSCOM Reg 350-1 & Active Duty Training for FORSCOM Units \\
\hline AR-95-20 & Contractor's Flight and Ground Operations \\
\hline UAS Platform User Manual & Most current version of the UAS Platform User Manual \\
\hline
\end{tabular}




\subsection{OPERATING PROCEDURES:}

\subsection{Ground Operations}

11.1.1. The Mission Commander (MC) will insure that all personnel comply with the requirements and stipulations of the Army Airworthiness Release, the FAA/DoD MOA, and/or an FAA COA.

11.1.2. A single, designated control station under control of the FO will be used for any mission planning.

11.1.3. Reprogramming or updating of flight controller software/firmware will be in accordance with the approved maintenance procedures.

11.1.4. Prior to implementation of any software updates the software security procedure will be used to prevent introduction of malicious code from infecting the control station and/or the air vehicle.

11.1.5. Software downloaded directly from the manufacturer's website and will be scanned for malicious software prior to connecting UAS aircraft to the control station and/or maintenance laptop. The contents of the control station and/or maintenance laptop as a whole will also be scanned regularly for malicious software.

11.1.6. Recharging of flight pack and auxiliary lithium-polymer (LiPo) batteries will be performed with LiPo-compatible chargers and according to best practices for safe charging of LiPo batteries.

11.1.7. In the event a new payload is being integrated, an electromagnetic compatibility (EMC) check will be completed on the ground prior to flight.

11.1.8. In the event a new payload is being integrated, a "center of gravity check" will be conducted and recorded prior to takeoff. Pilots will take extreme care upon initial takeoff to ensure the aircraft flight characteristics remain the same.

\subsection{Flight Scheduling}

The MC will schedule all flights in accordance with the Army Airworthiness Release, the FAA/DoD Memorandum of Agreement (MOA), and/or an FAA COA, Range Control, and/or local airfields in accordance with the deployed local operating procedures at that facility. 
11.3. Briefings and Risk Management

11.3.1. All UAS training flights are considered low risk due to the weight, limited speed, lack of flammable fuels, and the aircraft must be operated within line of sight (LOS) of the UAS FO and SO. The aircraft training flights are restricted to unpopulated areas where risk to people and property is minimal.

11.3.2. LOS needs to be maintained between the aircraft and the Mission Launch Location.

11.3.3. All UAS flights conducted for other than training must have a hazard analysis and a risk determination. The UAS FO will obtain briefings and implement the mitigations in accordance with the risk determination prior to any flight operations.

Briefings will be conducted by the MC.

11.3.4. UAS vehicles will be operated/piloted by government civilian employees.

\subsection{Weather}

11.4.1. UAS will not operate with weather conditions that exceed Visual Meteorological Conditions (VMC) in accordance with the FAA definition of VMC. The UAS will not operate beyond the following limits:
a. Ceiling less than 400 feet.
b. Visibility less than 1 statute mile.
c. Winds greater than 30 knots (35 mph).
d. Adverse weather to include rain/lighting.

11.4.2. The SO will monitor weather conditions throughout the flight and advise when conditions are approaching the conditions above.

\subsection{Operating/Safety Restrictions}

11.5.1. UAS is restricted for operational use to altitudes below $400 \mathrm{ft}$ above ground level (AGL) and within visual LOS of the FO. The UAS FO will never exceed the boundaries of the operation area established by the active restricted airspace limitations, or in accordance with the FAA/DoD MOA or FAA COA.

11.5.2. The UAS FO will comply with all safety requirements defined by the AWR, MOA, and/or COA plus additional site requirements. 
11.5.3. The UAS aircraft will not be operated within a prescribed safety standoff distance as determined by the hazard assessment of any flight crew personnel while in operation to minimize the risk to injury in the event of a mishap.

11.5.4. The UAS aircraft shall not be operated within a prescribed standoff distance, as determined by the hazard assessment of any non-flight crew personnel, during operation to minimize the risk of injury in the event of a mishap.

11.5.5. All UAS will be operated according to the correct version of the operator's manual for flight operations for each system.

\subsection{Frequency Management and Control}

11.6.1. The UAS aircraft operate using $2.4 \mathrm{GHz}$ (spread spectrum) for the radio control link and $5.8 \mathrm{GHz}$ (single channel) for each payload downlink. The UAS MC is the frequency control officer for each flight operational area. He/she is responsible for deconfliction of frequencies prior to operating the UAS.

11.6.2. The FO will insure the frequency for each operational area is correct prior to flight. He/she is responsible for de-conflicting the frequencies prior to operating the system.

11.6.3. Before each flight series, the FO will ensure that frequency allocation requirements for the operational area have been satisfied.

\subsection{Crew Coordination}

11.7.1. The $\mathrm{MC}$ is responsible to insure all personnel for the operation of the UAS must use clear communications to ensure safety. The UAS FO will rely on the Safety Observer to relay information in person, by cellular phone, and/or by radio (in which case radio operation training will be provided), as required. If the FO determines there is a problem with UAS while in autonomous GPS flight/hover, then he/she will assume full manual control of the UAS.

11.7.2. The UAS FO will use the most conservative approach when a disagreement exists.

11.8. Pre-Flight Checklist (performed by FO unless otherwise noted)

11.8.1. Assign SO and ASO. Have SO coordinate with ATC, Range Control, etc. for airspace use. 
11.8.2. Assess layout of test site with particular attention to overhead obstacles such as power lines. Identify alternate emergency landing site(s) in coordination with SO.

11.8.3. $\mathrm{MC}$ will confirm the flight plan with $\mathrm{FO}$ and SO.

11.8.4. Check all screws, propellers, connections, etc. for tightness and structural integrity.

11.8.5. Install fully charged flight pack batteries and auxiliary batteries (video links, cameras, etc.). Ensure that battery leads are secured away from the propeller arcs.

11.8.6. Confirm proper center of gravity by lifting the aircraft at the center hub with two fingers and look for excessive lean in any direction.

11.8.7. Confirm orientations of all antennas and double-check GPS compass for secure mounting.

11.8.8. Power-on payload (GoPro Hero, Thermal Camera Gimbal, etc.).

11.8.9. Power-on payload RC transmitter and test payload control.

11.8.10. Check Command and Control RC transmitter settings:

11.8.10.1 Mode: GPS

11.8.10.2 Rates (if applicable): High

11.8.10.3 Throttle: Down

11.8.10.4 Course: Off

11.8.10.5 Go Home in Standby

11.8.10.6 Gear (if applicable): Down

11.8.11. Power-on RC transmitter.

11.8.12. Confirm RC transmitter model set to correct model.

11.8.13. Power-on aircraft and wait for full GPS lock plus GPS home confirmation before disturbing the aircraft.

11.8.14. Confirm proper operation of telemetry (altitude and flight pack voltage) between transmitter and receiver. 
11.8.15. Check operation of payload camera(s) and video downlink(s) and confirm proper operation with control station. Press "record" on camera(s).

11.8.16. Clear the area of personnel and obtain clearance from SO for takeoff. Verbally announce "Clear" before starting the motors.

11.8.17. Start the motors and idle by moving the Command and Control $\mathrm{RC}$ transmitter sticks down and inward for 2 seconds or until the motors start, then release the sticks and apply $1 / 4$ throttle if necessary.

11.8.18. Test the flight controls by pitching and rolling to confirm the aircraft is leaning slightly in the direction of the flight stick.

11.8.19. Switch from Standby to Home and back to Standby to set the home position.

11.8.20. Verbally announce "Taking Off" to flight crew to relay intent to take off.

11.8.21. Verify GPS mode by noting the status light is still flashing purple.

11.8.22. Apply full throttle and Loiter/Hover at an altitude of approximately 10 feet and check for any unusual aircraft tendencies, noises, vibrations, etc.

11.8.23. Confirm proper operation of telemetry and payload downlink(s) at Control Station while in flight.

11.8.24. Execute flight plan.

11.9. Post-Flight Checklist (performed by FO unless otherwise noted)

11.9.1. Press "stop" on payload/camera(s) to conclude data acquisition.

11.9.2. Immediately check flight pack batteries and motors for excessive heat generation.

11.9.3. Disconnect all on-board batteries.

11.9.4. Power-down the RC transmitters.

11.9.5. Inspect the UAS for loose parts/connections, structural damage, etc.

11.9.6. Record flight description and data in flight log.

11.9.7. Request that SO contact ATC, Range Control, etc. upon completion of airspace use. 


\section{Annex A - Sample Hazard Analysis and Risk Assessment Worksheet}

Annex A should be completed in light of the organization's planned flight activities. Significant planning should be allotted to ensure all pertinent risks are identified, and to ensure those risks are addressed by mitigation or lessening measures. 


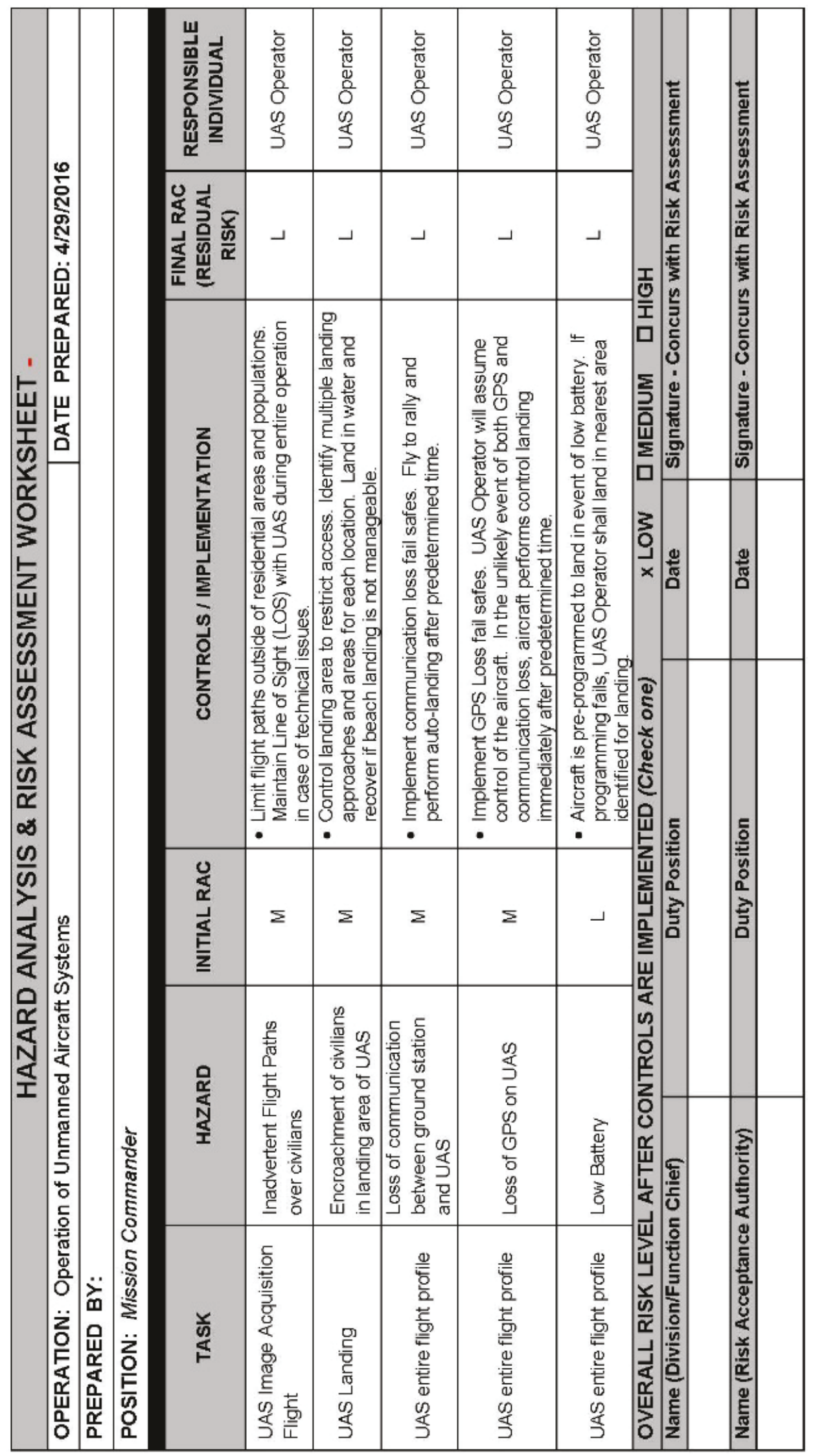

놀 


\section{Annex B: Checklist for Development of a Standing Operating Procedure}

(To be included as part of the SOP development, review and approval process. This checklist aids in the development of the whole AWR package and ensures that required documents are completed.)

SOP Title and Number: ORGANIZATION SUB-ORGANIZATION UAS SOP

Operation / Process: 1

SOP Preparer:

Date of Readiness for Review: 20160415

Gov't Management / Lead Reviewer:

Operator Reviewer:

Gov't Safety Reviewer:

Contractor Reviewer (as applicable): 


\begin{tabular}{|c|c|c|c|}
\hline General & Yes & No & $N / A$ \\
\hline $\begin{array}{l}\text { 1. Is the SOP written to eliminate, control, or reduce hazards and } \\
\text { associated risks and provide comprehensive safety protection? }\end{array}$ & $x$ & & \\
\hline $\begin{array}{l}\text { 2. Is the operation or task considered high risk, complex, or involve } \\
\text { hazardous materials and written with the appropriate level of safety } \\
\text { protection/requirements? }\end{array}$ & & & $\mathrm{x}$ \\
\hline 3. Does the SOP provide environmental protection? & $x$ & & \\
\hline $\begin{array}{l}\text { 4. Does the SOP adequately describe the purpose and methods for } \\
\text { the operation? }\end{array}$ & $x$ & & \\
\hline $\begin{array}{l}\text { 5. Can operating manuals, work plans, internal operating plans, } \\
\text { work instructions, field manuals (FMs), or other published docu- } \\
\text { ments be substituted for or supplement this SOP to reduce duplica- } \\
\text { tion and unnecessary effort? }\end{array}$ & & $x$ & \\
\hline $\begin{array}{l}\text { 6. Does this SOP involve the direct execution of mission and pro- } \\
\text { cesses and not administrative duties or excessive general safety in- } \\
\text { formation? }\end{array}$ & $x$ & & \\
\hline $\begin{array}{l}\text { 7. Does the Cover Sheet have adequate identifying information? } \\
\text { Specific areas to be included are: }\end{array}$ & $x$ & & \\
\hline a. Unique SOP number? & $x$ & & \\
\hline b. Date? & $x$ & & \\
\hline c. Operation or activity name? & $x$ & & \\
\hline d. Name of process or function? & $x$ & & \\
\hline e. Name of preparer with position/title and phone number? & $x$ & & \\
\hline
\end{tabular}




\begin{tabular}{|c|c|c|c|}
\hline $\begin{array}{l}\text { f. Signatures of responsible reviewers and management/safety authorities } \\
\text { with name, title and approval dates? }\end{array}$ & $x$ & & \\
\hline $\begin{array}{l}\text { 8. Did the direct managing authority over the operation/task prepare } \\
\text { this SOP? }\end{array}$ & $x$ & & \\
\hline $\begin{array}{l}\text { 9. Did experienced and/or qualified personnel with expertise for this } \\
\text { operation, process and/or equipment prepare this SOP? }\end{array}$ & $x$ & & \\
\hline $\begin{array}{l}\text { 10. Will the SOP be readily available to supervi- } \\
\text { sors/leads/safety/audit and operator personnel? }\end{array}$ & $x$ & & \\
\hline Writing the Standing Operating Procedure & Yes & No & $N / A$ \\
\hline 1. Was the work environment adequately addressed? & $x$ & & \\
\hline $\begin{array}{l}\text { 2. Were all required supplies and materials properly identified in de- } \\
\text { tail? }\end{array}$ & $x$ & & \\
\hline $\begin{array}{l}\text { 3. Has all required personal protective equipment (PPE) been iden- } \\
\text { tified? }\end{array}$ & & & $x$ \\
\hline $\begin{array}{l}\text { 4. Have all inherent hazards, types and risks been identified for the } \\
\text { operation and eliminated or mitigated by an adequate safety proce- } \\
\text { dure? Are the following specific areas of the SOP readily identifia- } \\
\text { ble: }\end{array}$ & $x$ & & \\
\hline a. Steps? & $x$ & & \\
\hline b. Processes? & $x$ & & \\
\hline c. Checks? & $x$ & & \\
\hline d. Precautions? & $x$ & & \\
\hline
\end{tabular}




\begin{tabular}{|c|c|c|}
\hline e. Warnings? & $\mathrm{x}$ & \\
\hline f. Cautions? & $\mathrm{x}$ & \\
\hline g. Protective equipment, clothing, guards, and barriers? & $\mathrm{x}$ & \\
\hline $\begin{array}{l}\text { 5. Are the beginning and end of a hazardous set of steps readily } \\
\text { identifiable? }\end{array}$ & $x$ & \\
\hline $\begin{array}{l}\text { 6. Have personnel from safety, environmental, logistics, quality as- } \\
\text { surance, fire, emergency operations, engineering, and production } \\
\text { input to the SOP where required? }\end{array}$ & $x$ & \\
\hline $\begin{array}{l}\text { 7. Are there provisions for a walk-through or run-through (inert for } \\
\text { explosive operations) of the steps for demonstration of safe opera- } \\
\text { tions prior to the actual planned operation or task? }\end{array}$ & & $\mathrm{x}$ \\
\hline a. Was it documented and verified? & & $\mathrm{x}$ \\
\hline $\begin{array}{l}\text { b. Was the supervisor/lead of the operation and safety present during the } \\
\text { demo? }\end{array}$ & & $\mathrm{x}$ \\
\hline $\begin{array}{l}\text { 8. Has a Job Hazard Analysis been conducted for each job type in } \\
\text { the operation? }\end{array}$ & & $x$ \\
\hline $\begin{array}{l}\text { 9. Has a hazard/risk assessment been developed in support of the } \\
\text { SOP? }\end{array}$ & $x$ & \\
\hline $\begin{array}{l}\text { a. Has the residual risk for the operation been clearly identified and accepted } \\
\text { by the appropriate authority? }\end{array}$ & $\mathrm{x}$ & \\
\hline b. Has a qualified safety professional reviewed and approved it? & $\mathrm{x}$ & \\
\hline $\begin{array}{l}\text { 10. Do each and all steps of the SOP direct the operator to accom- } \\
\text { plish its objective in a clear, concise, usable, and adequate manner } \\
\text { with an appropriate level of detail for a first time user? }\end{array}$ & $x$ & \\
\hline
\end{tabular}




\begin{tabular}{|c|c|c|c|}
\hline $\begin{array}{l}\text { 11. Are all of the steps of the SOP mission/process specific and not } \\
\text { a general safety management plan? }\end{array}$ & $x$ & & \\
\hline $\begin{array}{l}\text { 12. Are there provisions for safety critical sets of steps being vali- } \\
\text { dated by quality and/or safety? }\end{array}$ & $x$ & & \\
\hline $\begin{array}{l}\text { 13. Are equipment labels and markings cited adequately in the } \\
\text { SOP? }\end{array}$ & $x$ & & \\
\hline $\begin{array}{l}\text { 14. Does the SOP provide for prompt notification of required au- } \\
\text { thorities and management/safety in the event of emergencies or } \\
\text { other issues? }\end{array}$ & $\mathrm{x}$ & & \\
\hline $\begin{array}{l}\text { 15. Has a back-out plan (for High and Medium risk operations) in } \\
\text { case of an emergency been included? }\end{array}$ & $x$ & & \\
\hline $\begin{array}{l}\text { 16. Does the SOP index contain the SOP number, title, submitting } \\
\text { office, date, signature page, and review and approval date? }\end{array}$ & & & \\
\hline $\begin{array}{l}\text { 17. Was the SOP written in compliance with DA Pam } 385-10 \text {, } \\
\text { Chapter } 9 \text { ? }\end{array}$ & $x$ & & \\
\hline $\begin{array}{l}\text { 18. Has a Draft of the SOP been reviewed for completeness and } \\
\text { accuracy by required personnel prior to submittal for review? }\end{array}$ & $x$ & & \\
\hline Review-Concurrence-Confirmation of Use & Yes & No & $N / A$ \\
\hline $\begin{array}{l}\text { 1. Was the SOP reviewed by operating personnel and acknowl- } \\
\text { edged so via a written and signed statement? }\end{array}$ & $x$ & & \\
\hline $\begin{array}{l}\text { 2. Was the SOP reviewed, approved, and an acknowledgement } \\
\text { statement for sign-off by contractor management and safety over- } \\
\text { sight provided, as applicable? }\end{array}$ & $x$ & & \\
\hline
\end{tabular}




\begin{tabular}{|c|c|}
\hline $\begin{array}{l}\text { 3. Was the SOP reviewed, approved, and an acknowledgement } \\
\text { statement for sign-off by the required government supervi- } \\
\text { sor/lead/safety oversight authorities provided? }\end{array}$ & $x$ \\
\hline $\begin{array}{l}\text { 4. Have the qualifications of all operators to perform the task and } \\
\text { supervisors/leads as oversight of operations been validated? }\end{array}$ & $x$ \\
\hline $\begin{array}{l}\text { 5. Has an appropriate SOP review cycle been established com- } \\
\text { mensurate with the level of operation and personnel risk (a mini- } \\
\text { mum of one year for high and medium risk operations and two years } \\
\text { for low risk operations)? }\end{array}$ & $x$ \\
\hline $\begin{array}{l}\text { 6. Has the supervisor/lead in charge signed the supervisory } \\
\text { acknowledgement statement page of the SOP prior to first use, } \\
\text { when a change has been made to the SOP, or after the scheduled } \\
\text { review cycle (at least annually for high and medium risk operations } \\
\text { and if an operation or task is performed on a continuous basis)? }\end{array}$ & $x$ \\
\hline $\begin{array}{l}\text { 7. Has the operator signed the acknowledgement statement page } \\
\text { of the SOP prior to first use, when a change has been made to the } \\
\text { SOP, or after the scheduled review cycle (at least annually for high } \\
\text { and medium risk operations and if an operation or task is performed } \\
\text { on a continuous basis)? }\end{array}$ & $x$ \\
\hline $\begin{array}{l}\text { 8. Will the supervisor/lead use the SOP during training, oversight } \\
\text { and verification of operations and operators? }\end{array}$ & $x$ \\
\hline
\end{tabular}




\section{Annex C: Emergency Notification Procedures}

\subsection{EMERGENCY TELEPHONE NUMBER—911.}

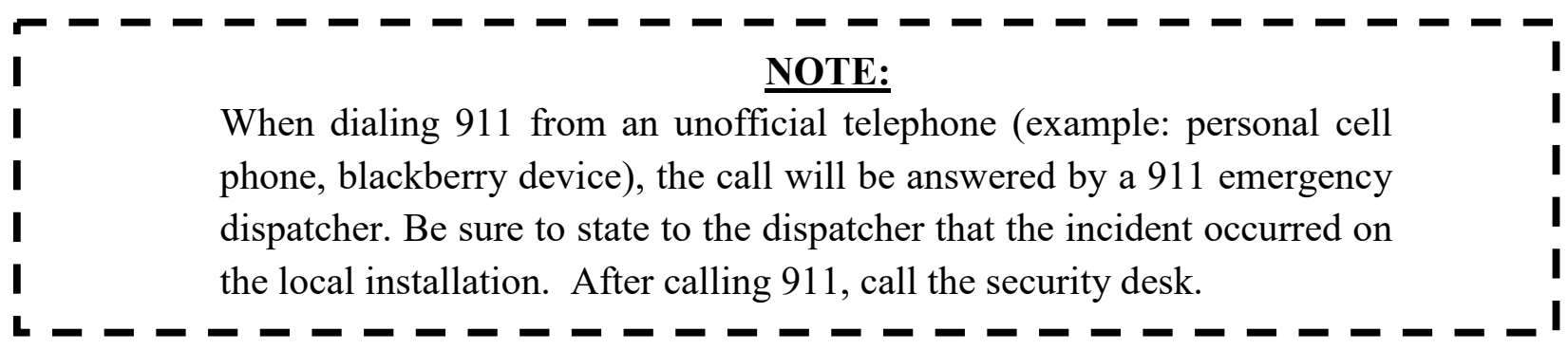

1.1. In the event of a serious injury or fatality, fire, or hazardous substance spill, immediately dial the Operational Location emergency telephone number-911.

1.2. When reporting an incident, provide as much information as possible to ensure adequate emergency response resources are dispatched; however, DO NOT WAIT until information is available before calling. The minimum information provided should include the following:

1.2.1 Where the incident occurred, such as the building number, room number and other details.

\subsubsection{Are there personnel injured? YES or NO}

1.2.3 If YES, provide operator with as much information as possible:

- Exact location of incident.

- Telephone number calling from and your name.

- What happened-FIRE, EXPLOSION, INJURY, etc.

- Number of individuals injured.

- Condition of victim(s) (if known)—conscious/unconscious, signs of life, etc.

- Is emergency medical assistance (First Aid) being providedCPR, First Aid, and AED.

1.2.4 (ONLY IF APPLICABLE) If the incident involves a FIRE with explosive materials, immediately evacuate personnel to a pre-determined "safe-haven" (as specified in the SOP), then using a 
telephone at a safe location, provide the operator with as much information as possible:

- Hazard Division (HD) of explosive materials involved-HD

- Estimated Net Explosive Weight (NEW) of explosive materials

- Location of other potentially explosive/flammable materials, e.g., flammable liquids, compressed gases, etc.

- Location and distance personnel have been evacuated too.

- Whether or not ALL personnel have been accounted for.

1.2.5 (ONLY IF APPLICABLE) If the incident involves a chemical spill or release, or fire involving hazardous chemicals, immediately evacuate personnel (with potential for exposure) away from building in an upwind direction, then using a telephone at a safe location, provide the operator with the following information:

- What hazardous chemical or substance was spilled (if known)?

- What is the estimated quantity of the spill (in pounds or gallons)?

- What was the time of the spill?

- Was the spill contained? How?

- What distance have personnel been evacuated too?

- Has the spill resulted in a fire?

- Have ALL personnel have been accounted for?

\subsection{NOTIFICATION PROCEDURES.}

2.1. After dialing 911, a Responsible Individual, Technician, or a designated person, will immediately notify the security desk and the following management and support staff (using the sequence of calling as indicated) that a serious incident has occurred and emergency response personnel have been notified: 
(a) UAS Program Manager (Name, Phone Number)

(b) Chief, Sub-Organization (Name, Phone Number)

(c) Safety Manager (Name, Phone Number)

2.2 A Responsible Individual (supervisor or lead) or a knowledgeable individual will provide whatever assistance required to the emergency response team in support of accessing the incident and determining the required protective posture level. Unless the accident area is deemed unsafe, emergency response personnel should be able to proceed directly to the accident location. If deemed unsafe (immediate life-threatening conditions) emergency response personnel may choose to stop at a safe distance to assess the incident before proceeding. Based on available information provided to the On-Scene Commander, he will initiate response actions as necessary to protect lives ( $1^{\text {st }}$ Priority) and property ( $2^{\text {nd }}$ Priority).

2.3 In the event an incident occurs during non-duty hours, the responding installation Emergency Response Team Supervisor should immediately notify the security desk, to alert the safety manager that an incident has occurred, and the required response to the incident IAW their Emergency Notification Procedures.

\subsection{EMERGENCY RESPONSE ACTIONS.}

\subsection{FIREFIGHTING. LET PROFESSIONALLY TRAINED} PERSONNEL FIGHT FIRES. DO NOT ATTEMPT TO FIGHT OR PUT OUT A FIRE IF ANY OF THE FOLLOWING CONDITIONS EXIST:

- YOU ARE UNSURE OF WHAT IS BURNING.

- THE FIRE IS SPREADING RAPIDLY.

- YOU DO NOT KNOW HOW TO SAFELY USE A FIRE EXTINGUISHER OR YOU DO NOT HAVE THE PROPER TYPE OF FIRE EXTINGUISHER.

- DENSE SMOKE IS BEING GENERATED FROM THE FIRE.

- THE FIRE MIGHT BLOCK YOUR MEANS OF EGRESS.

- $\quad$ YOUR INSTINCTS TELL YOU NOT TO DO SO. 
3.2. BUILDING EVACUATION. In the event of an emergency condition (defined as either a fire involving ammunitions and explosives (A\&E) or other hazardous substances, or a chemical spill/release), the on-site Responsible Individual (within the Facility) will immediately perform the following actions:

3.2.1 EVACUATE all personnel from the building and surrounding area (in an upwind direction) to a safe distance (location). Take any readily available first aid equipment-first aid kits, automated external defibrillator (AED), fire blankets, etc. Do not re-enter facility to obtain this equipment.

\subsubsection{IMMEDIATELY CALL—911 (Initiate Emergency Notifi- cation Procedures).}

\subsubsection{IMMEDIATELY INITIATE ACCOUNTABILTY}

PROCEDURES for all facility occupants (to include transients, visitors, and contractor-support personnel) to the best of your ability; stand-by to provide information as needed to the Emergency Response Team On-Scene Commander.

3.3. EMERGENCY FIRST AID. In the event emergency First Aid response is required, only those personnel trained in First Aid, cardiopulmonary resuscitation (CPR), and AED usage should provide care to victims (within their scope of training and capability) and remain with the victim until professional emergency medical response team can arrive on site. In the event the victim(s), others, or yourself are threatened by immediate life-threatening danger, e.g., spreading fire, chemical exposure, explosions, etc., all available and physically capable personnel will move the victim(s) to a safe area (up wind, behind a protective structure or other location / building, etc.). When moving a victim, use every possible measure to prevent further harm to the victim (spine or neck injuries). 


\section{Annex D: Environmental Protection Measures}

(SOLID \& HAZARDOUS WASTE MANAGEMENT)

\subsection{GENERAL}

1.1 All operation(s) contained in this SOP will conform to the environmental protection compliance requirements of EPA $40 \mathrm{CFR}$, AR-200-1, AR-200-2, and any local Regulation required by facility.

1.2 Operational procedure(s) contained in this document have been evaluated IAW AR 200-2 and determined that procedures and work tasks do not individually or cumulatively have a significant (negative) effect or impact on the "environment". Further, these procedures will not adversely affect "environmentally sensitive" resources or create any environmentally controversial change to existing environmental conditions on SUB-ORGANIZATION property.

1.3 All personnel conducting operational work tasks with the potential for a hazardous substance spill will be knowledgeable of the emergency response actions contained in the SUB-ORGANIZATION HAZMAT PLAN. Copies of the HAZMAT PLAN should be readily available in each work area where hazardous substances are present. In addition, the HAZMAT PLAN must be maintained on file and/or posted inside an operational facility Spill Prevention Control and Countermeasure (SPCC) Plan or the Installation Spill Contingency Plan (ISCP) for Oil and Hazardous Substances. The Plan should contain Emergency Notification Procedures and Emergency Response Actions that will be initiated in the event of a hazardous substance spill/fire.

1.4 All personnel working with hazardous materials (HM) and hazardous waste (HW) will be trained to standard IAW the Code of Federal Regulations (CFR) 29 Part 1910.1200 and 40 CFR Parts 262.34(a)(4) and 265.16(a)-(c). 


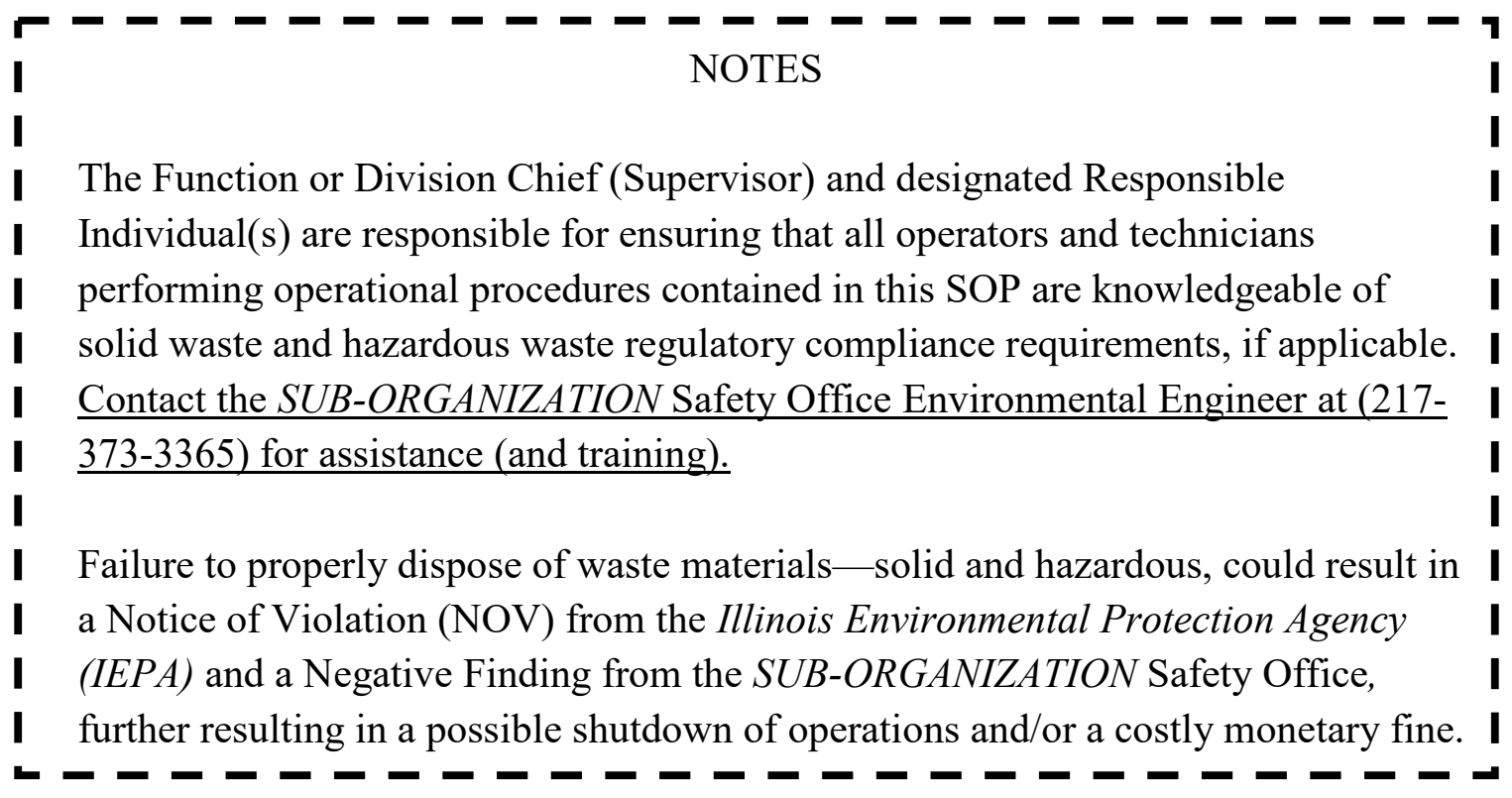

\subsection{HAZARDOUS MATERIALS / WASTE MANAGEMENT}

2.1 A Satellite Accumulation Area (SAA) should be established within the operational facility or work area for the accumulation of waste materials, as deemed necessary to support operations. If an SAA is deemed necessary, it will be maintained IAW EPA 40 CFR Part 262.34 Standards and any SUB-ORGANIZATION Safety Office Regulation.

2.2 All hazardous materials and waste (non-explosive / non-reactiveD003) will be reported IAW Local Site Environmental Management Division Regulation, Hazardous Materials/Waste Management Plan.

2.3 All solid waste (recyclable materials) that are not explosive (propellant) contaminated will be placed inside appropriate DRMO supplied waste bins, e.g., scrap metals, wood, etc., IAW DOD 4160.21-M-1.

2.4 All non-D003 waste streams will be sampled and analyzed to determine the proper waste hazard classification unless user knowledge and/or Material Safety Data Sheets are deemed adequate by the SUB-ORGANIZATION Safety Office. For assistance in determining waste stream classification, contact the SUB-ORGANIZATION Safety Office or the SUB-ORGANIZATION Safety Office Environmental Engineer: 


\subsection{PROPELLANT CONTAMINATED WASTE STREAMS}

3.1 Propellant contaminated waste streams (paper Kim-wipes, Q-tips, cheese cloth, other cleaning media) will be kept to a minimum, in approved, closed receptacles. "Brute" 10 gallon, Rubbermaid trash container (or other suitable container), with anti-static polyethylene liners (minimum 4-mil thick) will be used to contain propellant waste. The container will be marked, labeled or stenciled with "D003 REACTIVE [PROPELLANT CONTAMINATED] WASTE with the assigned Hazard Classification ( $\mathrm{HC}$ ) and Division as required, in 1-inch high white letters. Markings will be applied on the container lid and two locations on the side ( $180^{\circ}$ apart).

3.2 Propellant Contaminated Waste containers will be handled with special care. They will not be thrown about, tumbled, rolled, walked on, dragged, or slid on any surface or handled in such a way that may cause a hazardous condition.

3.3 The total weight of a Propellant Contaminated Waste container will not exceed 50 pounds. Containers weighing 50 pounds or less may be handled by a single person, provided proper lifting techniques are used.

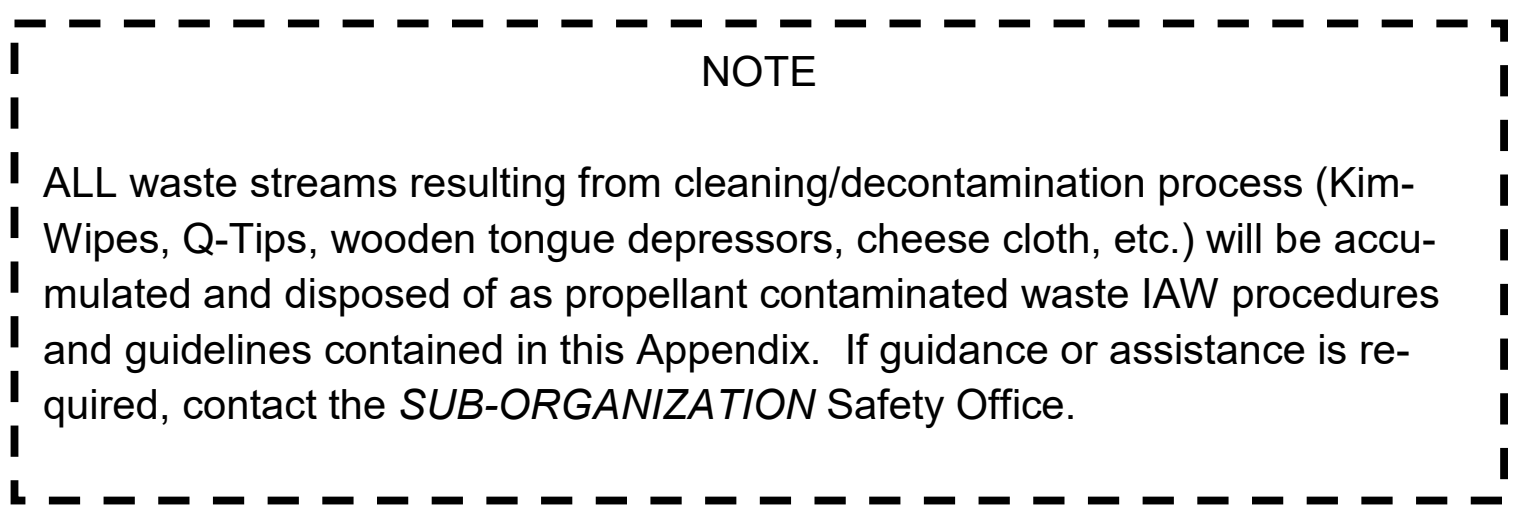

3.4 All materials potentially exposed to propellant or propellant residue will be deemed "propellant contaminated" unless a chemical analysis indicates otherwise. Individual bags of explosive contaminated waste may be placed inside a single waste container provided each bag has the air pressed out and sealed (polyethylene tape or Zip-Lock tie). INCOMPATIBLE WASTE 


\section{MATERIALS WILL NOT BE PACKAGED IN THE SAME}

CONTAINER. When the container is full, the outer bag will be sealed for turn-in.

3.5 Propellant Contaminated Waste will be removed from the work area at the end of each operating day. Waste containers, properly marked and closed will be transported (by approved HAZMAT vehicle and appropriately licensed operator) to an approved, less than 90-day A\&E waste storage area.

3.6 All solid waste (recyclable materials) that are not propellant contaminated will be placed inside appropriate Defense Reutilization and Marketing Office (DRMO)-supplied waste bins, e.g., scrap metals, wood, etc., IAW DOD 4160.21-M-1.

3.7 For additional assistance in hazardous waste stream handling, contact the SUB-ORGANIZATION Safety Office or the SUBORGANIZATION Safety Office Environmental Engineer. 


\section{Annex E - Mishap Action Plan}

\subsection{GENERAL}

1.1 This plan should be updated for every operational location and carried with the flight log book. The purpose of this plan is to provide roles and responsibilities for every member of the flight crew should an accident or mishap occur.

1.2 Emergency Flight Termination.

a. The authority to execute an emergency Flight Termination is Mission Commander (MC) responsibility. The MC delegates authority to the Flight Operator (FO), as deemed necessary.

b. The FO is responsible for notifying the Safety Observer (SO) and other flight crew in the area when executing an emergency flight termination.

c. The SO will notify the FO when intruder aircraft pose a threat to continued safe operations and advise the FO to execute and emergency Flight Termination.

d. Situations for emergency flight termination include but are not limited to:

(1) The aircraft appears to be in uncontrolled flight and/or impact outside of the operational consideration imminent.

(2) The aircraft fails to respond to any data link commands.

(3) Aircraft enter the operational area.

1.3 Uncontrolled flight outside of the approved operational area

a. The FO shall make every effort to return the UAS to the operational area.

b. If the aircraft continues in an uncontrolled state the:

(1) FO shall immediately notify the following authorities (based on operational location): 
- Air Traffic Control (ATC)

- Airspace manager

- All prescribed authorities per local requirements (police, fire department, etc.)

(2) MC if not present

(3) The SO shall continue to track the aircraft as long as possible and record positions.

1.4 Downed Aircraft inside and outside of the approved operational area

a. FO shall immediately notify the following authorities: (based on operational location)

- ATC

- Airspace manager

- All prescribed authorities per local requirements (police, fire department, etc.)

b. The FO shall notify the MC as soon as practicable.

c. The FO shall record the incident in writing as soon as possible.

\subsection{Downed Aircraft Recovery Team Procedures}

a. Downed Aircraft Recovery Team (DART) is comprised of key maintenance personnel to aid in the security and subsequent salvage operations of a downed aircraft. Key members include the $\mathrm{FO}, \mathrm{MC}$ if present, $\mathrm{SO}$ and trained maintenance flight crew members.

b. Control of personnel and crash site should be either the FO or MC.

c. The FO (if $\mathrm{MC}$ is not present) will immediately take actions necessary to protect personnel and equipment, secure all control stations, and notify the MC if not present of a UAS mishap. The FO will provide the date, time, and location of mishap and any known 
injury/damage information. Isolate all operators and crew involved with the flight. The $\mathrm{MC}$ or his representative will take charge of the scene and make all other notifications. Within 30 minutes of initial notification or arrival at mishap scene, the DART Leader or his representative will again contact the above individuals and provide preliminary mishap data for a mishap report.

d. Upon notification of an incident, the DART Leader assembles the team/equipment, moves to the crash site, and initially assists with security on the site, at the discretion of the FO. The mishap investigation begins once the site is secure.

e. DART Leader ensures communications and transportation requirements have been coordinated prior to commencing recovery operations. Immediately relay any deficiencies to the MC or supervisor for corrective action.

1.6 Actions at Mishap Scene (recommended actions be IAW AR 385-40, AR 95-23, and USARAK Reg. 385-1 as applicable)

a. DO NOT MOVE OR REMOVE ANYTHING. The mishap site must remain undisturbed and in its original state, until the end of the onsite investigation, or until the MC has given approval to remove an item.

b. Fire at the mishap site. Handle any incident involving a fire or crash of an aircraft with extreme care. Protect mission essential equipment and control the spread of any secondary fires.

c. Handling evidence at the mishap site. Exercise care not to disturb the evidence, as its location and physical orientation provide valuable data for the investigating team. Photograph all evidence, parts, and pieces at the mishap site where they lay.

(1) Initiate mishap/accident form.

d. Identify the personnel assigned to the DART and their knowledge about the UAS. Familiarity with the UAS is paramount to avoid unnecessary exposure to hazardous conditions at a mishap site.

e. Communications. Ensure the DART personnel have compatible communications equipment with the other support personnel, i.e., airfield personnel, fire, police, etc. Perform communication tests to determine functionality. 
f. Emergency Response Kit. Ensure the Emergency Response Kit is readily available to the designated DART personnel, and is in the flight crew vehicle prior to operations.

g. Fire procedures should be established for reporting and control procedures in the event of an aircraft fire of collateral fire associated by the mishap.

h. Fire response items should include all necessary firefighting equipment to team users.

i. Flight crew members who will be part of the fire response team should have training for the expected fire-fighting considerations.

1.7 Mishap Report. The mishap report should include the following:

a. Operational flight crew involved in the mishap

b. Location with details of crash location

c. Number and Type of Injuries

d. Was fire present or was there the possibility of fire?

e. Property damage to $3^{\text {rd }}$ parties and/or aircraft system

f. Fuel spillage / amount

g. Damage estimate to $3^{\text {rd }}$ parties and/or aircraft system

h. Aircraft total time and mission time

i. Local time of incident/mishap

j. Describe probable cause of how mishap occurred

k. Written and signed statements of all witnesses (all statements should be taken as soon as possible (ASAP) and witnesses should be segregated during the taking of statements)

I. Other pertinent information

m. Person reporting the mishap

n. Person filling out the mishap report

1.8 Guidance:

a. AR 95-23 Unmanned Aircraft System Flight Regulations 
b. DA-PAM 385-10 Army Safety Program

c. DA-PAM 385-40 Army Accident Investigation and Reporting 


\section{Appendix E: System Safety Management Plan Template/Sample}

The following template is reproduced here so that other users can see what is needed to create their own System Safety Management Plan. 
SYSTEM SAFETY MANAGEMENT PLAN

FOR

UNMANNED AIRCRAFT SYSTEMS (UAS)

Organization Name

Sub-Organization Name

Date Prepared:

$09 / 23 / 2016$

PREPARED BY: 
UNMANNED AIRCRAFT SYSTEMS SYSTEM SAFETY MANAGEMENT PLAN CONCURRENCE:

SIGNATURE

DATE

Name

Title

Name

Title

Name

Title 


\section{Introduction}

\subsection{Purpose}

This System Safety Management Plan (SSMP) establishes U.S. Army Construction Engineering Research Laboratory ( $S U B-O R G A N I Z A T I O N)$ small Unmanned Aircraft Systems (sUAS) life cycle management policies, objectives, and responsibilities for System Safety Program (SSP) execution. The SSP provides the means by which managers can identify and control hazards associated with all current and emerging UAS. The SSMP also describes the methodology for accepting residual risk.

\subsection{References}

a. Military Standard 882-C (MIL-STD-882C), 19 January 1993, System Safety

b. MIL-STD-882D, 10 February 2000, Standard Practice for System Safety.

c. Department of the Army Pamphlet 385-10 (DA Pam 385-10), 23 May 2008, Army Safety Program.

d. AR 385-10,23 August 2007, The Army Safety Program

e. Program Executive Officer (PEO), Aviation Policy Memorandums

(1) PEO Aviation Policy 05-12, 5 June 2006, Processing of System Safety Risk Assessments.

(2) PEO Aviation Policy 05-13, 21 November 2005, Processing of Hazard Executive Summaries.

(3) PEO Aviation Policy 05-15, 21 March 2006, Processing of Aircraft Findings and Recommendations

(4) PEO Aviation Policy 05-30, 23 September 2005, Project Manager System Safety Program Responsibilities

(5) PEO Aviation Policy 07-05, 10 January 2008, System Safety Risk Acceptance of Interim Risk Prior to Fielding.

(6) PEO Aviation Policy 08-03, 20 June 2008, System Safety Risk Management Process. 
f. Memorandum, Office of the Chief of Staff, DACS-SF, 26 March 1991, Application of Hazard Management.

g. Memorandum of Instruction (MOI), AMSAM-SF, 25 Jun 2007, Safety Risk Management Application and Accountability

\subsection{Scope}

This SSMP will be executed by the SUB-ORGANIZATION, Unmanned Aircraft Systems Team, who will ensure requirements are appropriately levied on supporting Government organizations and on prime contractors via contractual statements of work. The SSMP establishes the methods by which the program manager monitors, evaluates and ensures the execution of the SSP.

\subsection{System Safety Program Goal}

The UAS safety program goal is to identify, control and/or eliminate safety risks associated with system operation, maintenance, and support as defined in approved operational requirements and the system specifications.

\subsection{Policy}

It is the SUB-ORGANIZATION's policy to conduct the following at the Program Manager level:

a. Search continuously and systematically for potential system hazards and to assess their risk in terms of credible severity and probability.

b. Use a System Safety Working Group (SSWG) Team approach to risk management.

c. Resolve hazards in a time frame commensurate with the level of risk and program resources.

d. Eliminate hazards through engineering solutions, where possible, rather than implementing procedural controls.

e. Identify and document hazards and resultant actions in a tracking system through final resolution. 
f. Exercise, when warranted, low level-residual risk acceptance and process high-level residual risks within SUB-ORGANIZATION, in consultation with AMRDEC, IAW guidance provided.

g. Assist the User in defining materiel and operational constraints, and risks associated with proposed local mission or material changes as an interface with their mission risk assessment process.

\subsection{Definitions}

The following definitions are consistent with MIL-STD-882 and AR 385-10, and are tailored to fit the needs of the SUAS SSP:

a. Hazard - Any real or potential condition that can cause injury, illness, or death to personnel; damage to or loss of a system, equipment or property; or damage; or damage to the environment.

b. Hazardous Material - Any substance that, due to its chemical, physical, or biological nature, causes safety, public health, or environmental concerns that would require an elevated level of effort to manage.

c. Life Cycle - All phases of the system's life including design, research, development, test and evaluation, production, deployment (inventory), operations and support, and disposal.

d. Mishap - An unplanned event or series of events resulting in death, injury, occupational illness, damage to or loss of equipment or property, or damage to the environment.

e. Mishap Risk - An expression of the impact and possibility of a mishap in terms of potential mishap severity and probability of occurrence.

f. Residual Mishap Risk - The remaining mishap risk that exists after all mitigation techniques have been implemented or exhausted, in accordance with the system safety design order of precedence.

g. Safety - Freedom from those conditions that can cause death, injury, occupational illness, damage to or loss of equipment or property, or damage to the environment.

h. System Safety - The application of engineering and management principles, criteria, and techniques to achieve acceptable mishap risk, within the constraints of operational effectiveness and suitability, time, and cost, throughout all phases of the system life cycle. 
i. System Safety Engineering - An engineering discipline that employs specialized professional knowledge and skills in applying scientific and engineering principles, criteria, and techniques to identify and eliminate hazards, in order to reduce the associated mishap risk.

j. System Safety Management - An element of program management that establishes the system safety program requirements and ensures the planning, implementation, and accomplishment of tasks and activities to achieve system safety consistent with the overall program requirements.

k. System Safety Program (SSP) - The combined tasks and activities of system safety management and system safety engineering that enhance operational effectiveness by satisfying the system safety phases of the system life cycle.

1. System Safety Program Plan (SSPP) - A formal document prepared by the contractor that describes the planned safety tasks required to meet contractual system safety requirements. It includes organizational responsibilities, methods of accomplishment, milestones, depth of effort, and integration with other program engineering and management activities and related systems.

m. System Safety Working Group (SSWG) - An assembly of primary and associate members organized and chartered to assist the PM in achieving system safety objectives.

\subsection{Acronyms}
a. AAAR
Abbreviated Aviation Accident Report
b. AAE
Army Acquisition Executive
c. $\mathrm{AMC}$
Army Materiel Command
d. $\mathrm{AR}$
Army Regulation
e. AMRDEC
Aviation and Missile Research Development and Engineering Center
f. AMCOM
Aviation and Missile Command
g. AWR
Air Worthiness Release
h. CHPPM
Center for Health Promotion and Preventive Medicine
i. $\mathrm{CRC}$
Combat Readiness Center
j. DA
Department of the Army 


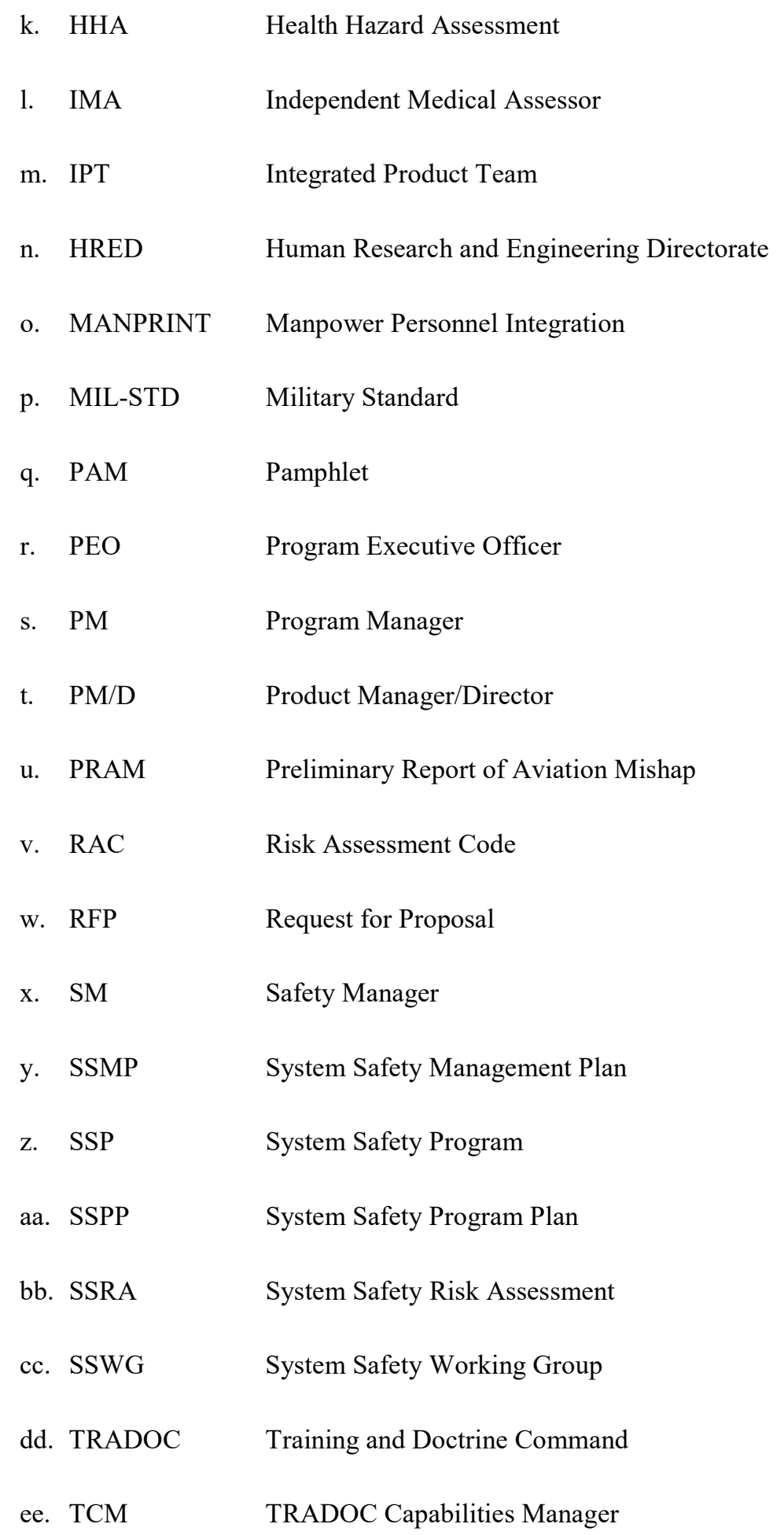




\section{Organizations and Responsibilities}

\subsection{General}

The Program Manager (PM) has overall responsibility for ensuring system safety throughout a system's life cycle. A formal SSWG will be established and will convene periodically (at least once per annum) during the life of the system to ensure appropriate coordination of the system safety concerns and to assist in identification, coordination, tracking, and correction of the system safety hazards. The SSWG will consist of primary and associate members selected to represent their respective activities. The PM or Safety Manager (SM) and SSWG primary members will handle day-to-day system safety activity. Findings and conclusions of the SSWG will be provided as recommendations to the Program Manager.

\subsection{Integration of Associated Disciplines}

a. Integrated Product Team (IPT). System safety specific testing issues and test data will be exchanged to assure hazards are identified and resolutions verified. The general safety of the test effort will also be reviewed. The Safety Manager is responsible for providing system safety documentation required by test activities.

b. Environmental. The system safety program may identify environmental issues during the hazard analysis and resolution process. System safety will coordinate with the appropriate Regulatory Office to hand off environmental hazards to the proper agency.

\subsection{Responsibility}

Individual and organizational responsibilities to achieve the required safety policy are described in the following paragraphs.

\subsubsection{Program Manager}

The Program Manager will:

a. Designate or carry out the duties of SM and delineate, delegate or carry out appropriate SSP implementation authority.

b. Establish and charter the necessary SSWGs (Appendix A). 
c. Ensure that applicable system safety engineering and management instructions, regulations, specifications, and standards are implemented with contract statements of work and system specifications.

d. Ensure that specific SSP objectives, requirements and funding are incorporated into planning and execution documents.

e. Assign budget and manpower resources to accomplish system safety management tasks required by Army regulation and this SSMP.

f. Ensure that hazard analyses are performed, documented, and reviewed IAW Army regulation policy.

g. Ensure that hazard risk management and residual risk acceptance is accomplished IAW Army regulation and PEO Aviation policy.

h. Establish decision-authority levels for the acceptance of residual risk IAW PEO Aviation policy.

i. Establish and maintain a documentation system (hazard tracking and/or SSRA files) for risk acceptance decisions.

j. Identify at each milestone review the hazards, residual risks, risk acceptance recommendations, or provide hazard resolution plans and status.

k. Establish government and contractor relationships and interactions that will ensure positive contributions to and an understanding of contractual system safety requirements.

1. Establish procedures for tracking system safety performance.

$\mathrm{m}$. Ensure that a formal hazard tracking system is established and adopted, and develop procedures for incorporating integrated human factors and health safety data identified by the cognizant disciplines throughout the project life.

n. Ensure that a safety assessment is conducted for all change proposals to confirm that new hazards are not introduced by design changes.

\subsubsection{Safety Manager}

In the absence of the Program Manager, each SM will:

a. Monitor the contractor SSP. 
b. Develop a tracking methodology for tracking hazards, findings and recommendations to final resolution; the final resolution being a completed formal software or a hardware change, or an approved system safety risk assessment.

c. Prepare and present a formal System Safety Risk Assessment (SSRA) in accordance with AR 385-10 for accepting residual risks categorized as medium or high.

d. Review all relevant Hazard Reports and Failure Reports to identify safety concerns.

e. Coordinate and manage the SSWG as Chairperson.

f. Develop a SSWG meeting schedule.

g. Coordinate all activities to ensure that cognizant contractor, Government, and field personnel are kept abreast of current activities relevant to system safety.

h. Ensure that all safety information bulletins and messages are routed to the appropriate personnel.

i. Maintain all relevant archival documents including, but not limited to, hazard tracking, deliverable safety reports, and meeting minutes.

j. Assure all development or integration contracts and applicable delivery orders contain the optimum system safety performance requirements in accordance with the philosophy of this plan.

k. Ensure that system safety documents required for milestone decision reviews are generated and provided for use in the review package.

1. Ensure that Hazard Tracking Records are reviewed prior to each System Safety Working Group meeting to determine any change in hazard status, and provide resulting conclusions and recommendations to the group.

m. Prepare Risk Determinations in the format of SSRAs, but requiring only PM signature, when a document is needed to communicate but not accept risk, e.g., to support safety messages.

n. Prepare and staff a Risk Acceptance Memorandum for Record to accept interim risk where an SSRA is not appropriate, such as for test. 
2.3.3 Aviation and Missile Research, Development, and Engineering Center (AMRDEC):

The Aviation Engineering Directorate (AED) and other directorates, as applicable, will provide a description of hazards identified during AWR process. They will also apply their expertise toward resolution action identification and implementation. AED will be represented as member(s) of the SSWG.

\subsubsection{Contractor System Safety Support}

The prime contractor will execute the contracted statement of work, which includes participation as a primary SSWG member and participation in the ITP.

\section{Risk Management}

Systematic analysis processes will be used to identify potential hazardous conditions. Analyses are not restricted to MIL-STD-882 processes but will also include reliability, human factors and environmental methods. All means will be used to

proactively identity hazards before related mishaps occur in the field. Priority will be given to identification before a design is finalized. The basic analysis steps are:

a. Perform a systematic assessment chosen to achieve the desired outcome.

b. For each identified hazard, determine the most serious credible outcome in terms of severity and probability and assign a risk assessment code.

c. For hazards that cannot be completely eliminated, for whatever reason, perform risk acceptance procedures commensurate with the residual risk level.

\subsection{Hazard Severity Categories}

The MIL-STD-882 hazard severity categories assess the consequences of the hazard outcome. Mishap severity categories provide a qualitative and/or quantitative measure of loss that could result from personnel error, environmental conditions, design inadequacies, procedural deficiencies, or component failure/malfunction. MIL-STD-882, Revision C added the degree of environmental damage to the definitions. Only system safety oriented environmental issues will be tracked under 
this system safety program. The environmental severity definitions are provided as guidance. NOTE: Severity codes are in Arabic numerals to coincide with the hazard tracking system database input requirements. MIL-STD-882 uses Roman numerals. They are interchangeable.

a. Category 1 -Catastrophic: Could result in death, permanent total disability, or irreversible severe environmental damage that violates law or regulation.

b. Category 2- Critical: Could result in severe injury or severe occupational illness (permanent partial disability), or reversible environmental damage causing a violation of law or regulation.

c. Category 3 -Marginal: Could result in minor injury or minor occupational illness (no permanent effect) resulting in one or more lost work days(s), system loss (loss of $\$ 20 \mathrm{~K}$ or more, but less than $\$ 200 \mathrm{~K}$ ), or minor environmental damage (without violation of law or regulation where restoration activities can be accomplished).

d. Category 4 -Negligible: Could result in less than minor injury or occupational illness (no lost workdays), or minor system damage (less than $\$ 20 \mathrm{~K}$ ), or less than minor environmental damage.

\subsection{Hazard Probability Levels}

The hazard probability will reflect the qualitative and/or quantitative expectation that the potential mishap, at the credible severity, will occur during operation at the system level. Refer to table 1 .

NOTE: There will be times when an additional probability should be referenced to show a high risk in relation to a specific phase or environment. This can be done in the hazard narrative with an explanation of the specific condition. For example, a specific maintenance hazard may have a very low hazard probability due to very infrequent performance. However, if the activity is required, there is a very high probability of injury. Without an additional condition specific reference point, the actual high-risk activity would be missed. 
Table 1. Probability level, description and anticipated reasonable expectation of occurrence.

\begin{tabular}{|l|l|l|l|l|}
\hline Level & Description & $\begin{array}{l}\text { Individual Item } \\
\text { (qualitative) }\end{array}$ & $\begin{array}{l}\text { Fleet or Inventory } \\
\text { (qualitative) }\end{array}$ & $\begin{array}{l}\text { Probability } \\
\text { (mishaps per 10,000 flight } \\
\text { hours) }\end{array}$ \\
\hline A & Frequent & $\begin{array}{l}\text { Likely to occur } \\
\text { often in the life of } \\
\text { an item. }\end{array}$ & Continuously experienced. & $\begin{array}{l}\text { Greater than 1000 hours } \\
\text { (expected to occur no more } \\
\text { than 1 in 10 flight hours). }\end{array}$ \\
\hline B & Probable & $\begin{array}{l}\text { Will occur several } \\
\text { times in the life of } \\
\text { an item. }\end{array}$ & Will occur frequently. & $\begin{array}{l}\text { Less than or equal to 100 } \\
\text { and greater than 10 hours } \\
\text { (expected to occur no more } \\
\text { than 1 in 100 flight hours). }\end{array}$ \\
\hline C & Occasional & $\begin{array}{l}\text { Likely to occur } \\
\text { sometime in the } \\
\text { life of an item. }\end{array}$ & $\begin{array}{l}\text { Will occur several times. } \\
\text { Oess than or equal to 10 } \\
\text { hours (expected to occur } \\
\text { no more than 1 in 1,000 } \\
\text { flight hours). }\end{array}$ \\
\hline D & Remote & $\begin{array}{l}\text { Unlikely but } \\
\text { possible to occur in } \\
\text { the life of an item. }\end{array}$ & $\begin{array}{l}\text { Unlikely, but can } \\
\text { reasonably be expected to } \\
\text { occur. }\end{array}$ & $\begin{array}{l}\text { Less than or equal to 1 } \\
\text { hour (expected to occur no } \\
\text { more than 1 in 10,000 } \\
\text { flight hours). }\end{array}$ \\
\hline
\end{tabular}

\subsection{Risk Assessment Code (RAC)}

The RAC is the combination of the severity category and the probability level, written as a number and a letter (refer to table 2). The RAC may be changed but the change must be justified and documented in the Hazard Tracking Record, or suitable supporting document. An initial RAC is assigned upon hazard identification and may change as the hazard is better defined or mitigation actions are taken. The goal is to complete the mitigation action by bringing the probability to zero. RACs for hazards with no residual risk requiring acceptance are described in paragraph $3.6 \mathrm{~g}$. 
Table 2. Table illustrating RAC matrix for determining risk assessment.

\begin{tabular}{|l|l|l|l|l|}
\hline & Frequent (A) & Probable (B) & Occasional (C) & Remote (D) \\
\hline Catastrophic (I) & High Risk & High Risk & High Risk & High Risk \\
\hline Critical (II) & High Risk & High Risk & High Risk & Medium Risk \\
\hline Marginal (III) & Medium Risk & Medium Risk & Medium Risk & Low Risk \\
\hline Negligible (IV) & Low Risk & Low Risk & Low Risk & Low Risk \\
\hline
\end{tabular}

Table 3. Command Authority for Risk Reporting and Acceptance.

\begin{tabular}{|l|l|l|l|l|}
\hline $\begin{array}{l}\text { RISK ASSESSMENT } \\
\text { CODE }\end{array}$ & LEVEL & PRIORITY & AUTHORITY & LEVEL \\
\hline IA-ID, IIA-IIC & HIGH & URGENT & $\begin{array}{l}\text { SUB- } \\
\text { ORGANIZATION }\end{array}$ & Director \\
\hline IID, IIIA-IIID & MEDIUM & CRITICAL & $\begin{array}{l}\text { SUB- } \\
\text { ORGANIZATION }\end{array}$ & $\begin{array}{l}\text { Deputy } \\
\text { Director }\end{array}$ \\
\hline IIID, IVA-IVD & LOW & ROUTINE & $\begin{array}{l}\text { SUB- } \\
\text { ORGANIZATION }\end{array}$ & Division Chief \\
\hline
\end{tabular}

\subsection{Risk Resolution}

a. Once a hazard has been identified and a RAC assigned, a decision will be made concerning what action will be taken to remedy the hazard. Hazards will be addressed according to priority of table 2 . The SSWG will identify potential methods for controlling or eliminating a hazard and estimate the expected effectiveness of each method. The SSWG will provide a written report/meeting minutes to the PM setting forth the risk assessment results and the hazard control recommendations.

b. Specific conditions required for recommending a hazard as "closed":

(1) Risk resolution by reclaiming parts- for example, searching for a lot of suspect parts by serial number:

(a) Safety message transmitted to locate and provide disposition instructions for parts, with required feedback from all addressees. If all parts are not located, then, 
(b) Second safety message as a follow-up, if required. If all parts are not located, then,

(c) Issue a directive affecting the Operating and/or Maintenance Manual issued to perform continuous inspection for specific parts or components.

(2) Risk reduction through the establishment of a permanent inspection intervalfor example, an addition to inspection checklists:

(a) Safety message announcing the change and requiring the inspection.

(b) Verified Technical Manual change published.

(3) Low level hazard risk reduction through a onetime inspection:

(a) Safety message.

(b) Verify that all affected serial numbered systems have log books updated to require the inspection.

(4) Medium or High level hazard risk reduction by one time inspection:

(a) Safety message requiring a report of inspection completion and/or condition found from every affected serial numbered system, within a specific suspense, as determined appropriate for the circumstances.

(b) Direct follow-up on all non-reported system serial numbers and from depot locations.

(c) Directive affecting the Operation and/or Maintenance Manual issued to perform continuous inspection for specific parts or components.

(5) Risk resolution by material change Modification Work Order or other forced retrofit program:

(a) An approved modification program.

(b) Funding approved and in place.

(c) System Safety Risk Assessment approved to accept interim risk, if required. 
(6) Risk reduction by attrition:

(a) An approved program decision acknowledging that tracking the materiel change is not necessary.

(b) An approved System Safety Risk Assessment to accept any interim risk, if required.

(c) Closure at zero risk may not be possible since completion is open ended.

(7) System Safety Risk Assessment:

(a) The SSRA is approved and the course of action, if any, is verified.

(b) An SSWG decision that SSRA actions do not require monitoring. (For example, a time limit is placed on the action required.)

3.5 Risk Acceptance

a. Materiel Developer Risk Acceptance

(1) Hazard resolution recommendations should serve as the initial documentation for a risk-acceptance decision. Table 3 defines the Command to which each hazard must be reported, and the decision authority for accepting the risk associated with each hazard.

(2) The consequences of risk acceptance of the proposed configuration and alternative actions should be stated quantitatively when practical. The decision to accept the risk shall also consider impact on schedule, operational effectiveness, and other factors.

b. Interface with User Mission Risk Assessment Actions.

(1) User/Operator Risk Assessments are encouraged.

(2) When the User requests a deviation from established configuration or procedures, the PM will address the request, coordinating directly with the requester and User Representative. 


\subsection{Hazard Tracking}

a. A hazard tracking system will be implemented by the PM to track all identified hazards. An example Hazard Tracking Record is at Appendix B.

b. The status of a hazard will be listed as "proposed" when it is submitted from any source and until opened or deleted by the SSWG or PM.

c. A hazard is in the "open" status when the SSWG or PM formally acknowledges it.

d. The "monitor" status identifies a category of open hazards that is assigned by the SSWG or PM to hazards which are awaiting test completion for better definition of the hazard, hazards awaiting test for substantiation of corrective action, hazards which are based on isolated events and more data is needed to justify corrective action/hazard closure, or hazards for which corrective action has been defined and is in progress.

e. The "recommended closed" status applies to hazards which the SSWG or PM has recommended to be closed.

f. The status of a hazard will be listed as "closed" only if the decision authority (Table 3-2) has given written approval of the final risk category (signed SSRA for medium or high risk), or the risk has been eliminated. The Hazard Tracking Record (Appendix B) will provide closure criteria.

g. In the Hazard Tracking Record database, hazards closed with no residual risk will receive 0-A (zero A), 0-R (zero R), or 0-S (zero S) codes. Previously accepted or closed hazards will be evaluated periodically (at least semi-annually) for changes in risk. The SSWG will approve recoding and the PM will be notified through the meeting minutes. Open or Monitor hazards may be recommended for zero residual risk closure with SSWG and PM approval. The Hazard Tracking Record will be updated to show the date of the review, the justification for RAC assignment, the action taken with the PM, and the final severity and probability. RACs for hazards with no residual risk will be assigned as follows:

(1) 0-A (zero A)- hazards which are no longer valid due to errors, superseded by other hazards and/or similar actions will be administratively coded 0-A and the action taken section appropriately annotated. 
(2) 0 -R (zero-R)- hazards eliminated by a materiel/design change, to include a life or overhaul time change.

(3) 0-S (zero-S)- hazards with no residual risk requiring acceptance by virtue of the design meeting or exceeding all applicable consensus and/or military standards and being operated in an environment consistent with those standards, i.e., being operated with published procedures that may include operating limitations, field inspections, and maintenance procedures.

h. Hazards within the "Low Risk" RAC may be closed without a formal System Safety Risk Assessment. The SSWG will recommend closure to the PM as part of the SSWG briefing and/or minutes. PM signature on the minutes shall constitute risk acceptance. The SSWG recommendation and reference to the signed minutes shall be noted in the Hazard Tracking Record.

i. The mishap, Equipment Improvement Recommendation and Quality Deficiency Report experience will be reviewed annually to determine whether or not previous risk management decisions should be reevaluated. 


\section{Annex A: \\ Program Manager \\ System Safety Working Group Charter}

\section{PURPOSE}

Establish a technically qualified advisory group for the Program Manager on system safety management as a means to enhance the safe design, sustainment and operation of the system and its mission and kits.

\section{MISSION}

The mission of the System Safety Working Group (SSWG) is to function as an element of project management and actively identify and assess hazards, monitor system safety task accomplishment, identify system requirements, organize and coordinate interfacing functions, and provide timely and effective recommendations to the PM.

\section{AUTHORIZATION}

Small Unmanned Aircraft Systems, SUB-ORGANIZARION.

\section{REFERENCES}

a. AR 385-10, The Army Safety Program, 23 August 2007.

b. DA PAM 385-10, Army Safety Program, 23 May 2008.

c. DA PAM 385-16, System Safety Management Guide, 4 September 1987.

d. MIL-STD-882D, System Safety Program Requirements, 10 February 2000.

\section{TASKS}

e. Oversee the log of system safety hazards, provide appropriate risk assessments, and track resolution actions. Recommendations to close hazards will be made to the PM. Hazard closure shall be in accordance with the current risk management procedures defined in the SUB-ORGANIZATION SUAS System Safety Management Plan 
f. Respond to requests by the PM for recommendations on program matters impacting system safety.

g. Coordinate with appropriate activities to identify and evaluate those areas in which system safety implications exist.

h. Provide recommendations for establishing or revising system safety requirements.

i. Identify and tailor SUB-ORGANIZATION SUAS documentation applicable to an effective system safety program. Tasks are:

(1) Assure integration of tailored system safety requirements into $S U B$ ORGANIZATION SUAS concerned contracts.

(2) Maintain the System Safety Management Plan and the System Safety Working Group Charter.

(3) Assure that safety issues for test and evaluation are identified and integrated into the appropriate test strategy and plans.

(4) Coordinate system safety documentation required for materiel acquisition milestone decisions.

(5) Assure corrective and/or acceptance action is taken on all hazards, particularly medium and high risk hazards.

(6) Analyze mishap/incident data for indications of new hazards and for the degree of success with previous assessments and resolution actions.

(7) Review the status of open hazard corrective actions, including accident findings and SSWG Action Items.

\section{OPERATION}

\section{Membership:}

(Note: Contractor members will be excluded from participation upon request of the Chairman when the potential for conflict of interest exists, 
proprietary information is discussed, or for other reasons when the propriety of their presence is questioned. The meeting announcement will, when possible, reflect any restrictions.)

a. The SSWG will be Chaired by the PM, or his/her designee.

b. Primary (regular) members are required to attend each meeting to successfully fulfill the purpose of the group. Primary members are the representatives of the following organizations:

(1) SUB-ORGANIZATION SUAS Team

(2) U.S. Army Aviation and Missile Research, Development, and Engineering Center (AMRDEC), Aviation Engineering Directorate (AED).

c. Advisory Members are representatives of other organizations, agencies, contractors, suppliers, etc., who will be invited to participate on an as-required basis.

d. Changes to the System Safety Working Group member organizations (primary members) require PM approval. Changes of advisory and associate member organizations will be made as required and approved by the co-chairperson.

\section{e. Meetings:}

(1) System Safety Working Group meetings will be held to work on individual issues and will usually include in-depth informational discussions of relevant system safety issues and advancements. Tasking will be recorded on Action Item sheets and managed by the PM to completion.

(2) Primary SSWG member meetings shall also be held at the call of the SM to assist in identification, definition, assessment, and resolution of immediate system safety issues. These meetings will normally be held telephonically and are not required to be concurrent conversations. These meetings will be an adjunct to the PM management team and, with the exception of new hazard list or tracking system input, will be reported under their procedures.

f. Administration: 
(1) Agenda items may be proposed by any organization but will be approved by the Safety Manager.

(2) Minutes will be prepared for formal SSWG meetings. A summary of key issues, action items and action agencies will be briefed prior to SSWG meeting termination to assure complete understanding and accuracy.

(3) SSWG recommendations will include minority opinions as applicable.

[NOTE: The following pages reproduce appendices to the safety system document.\} 


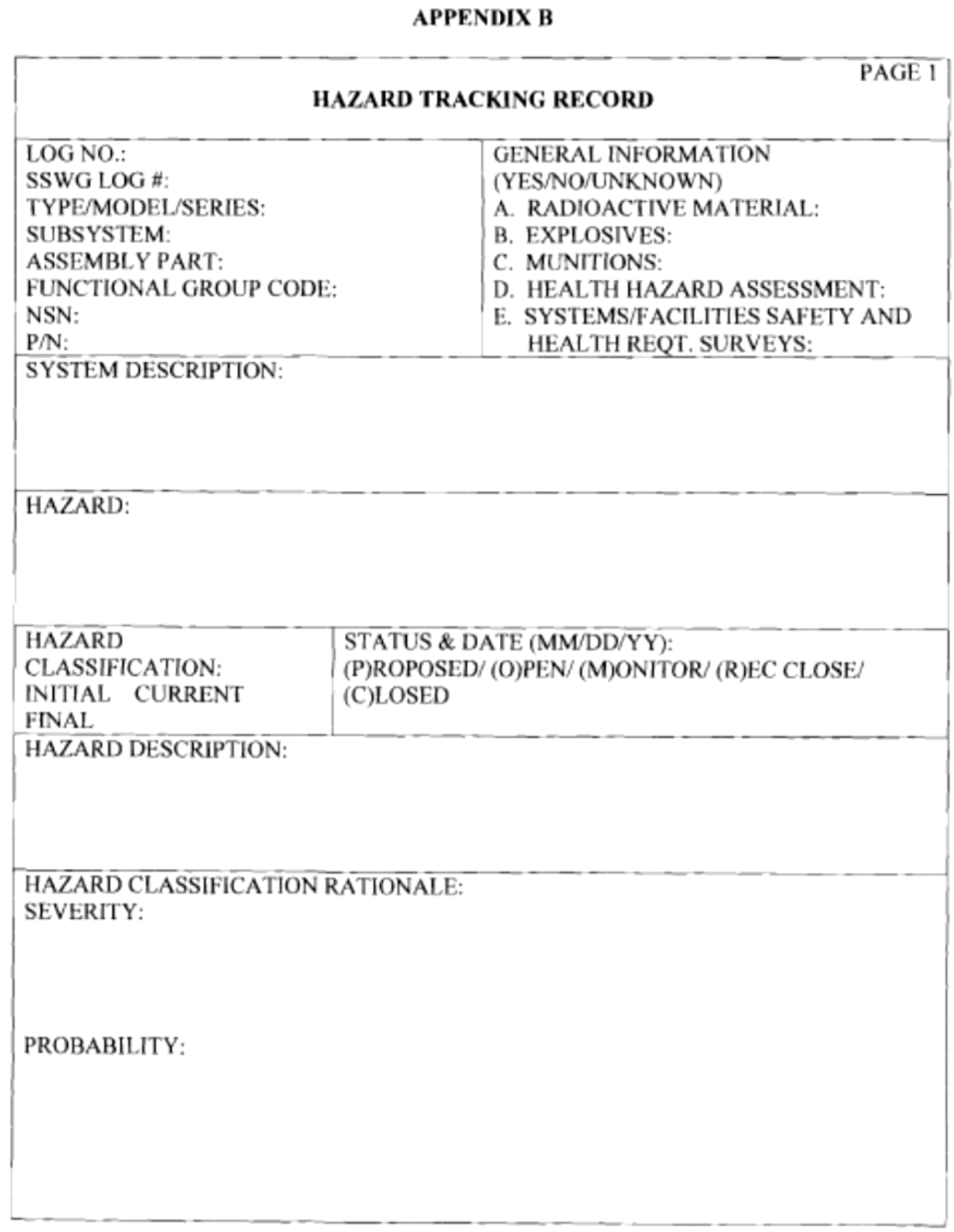




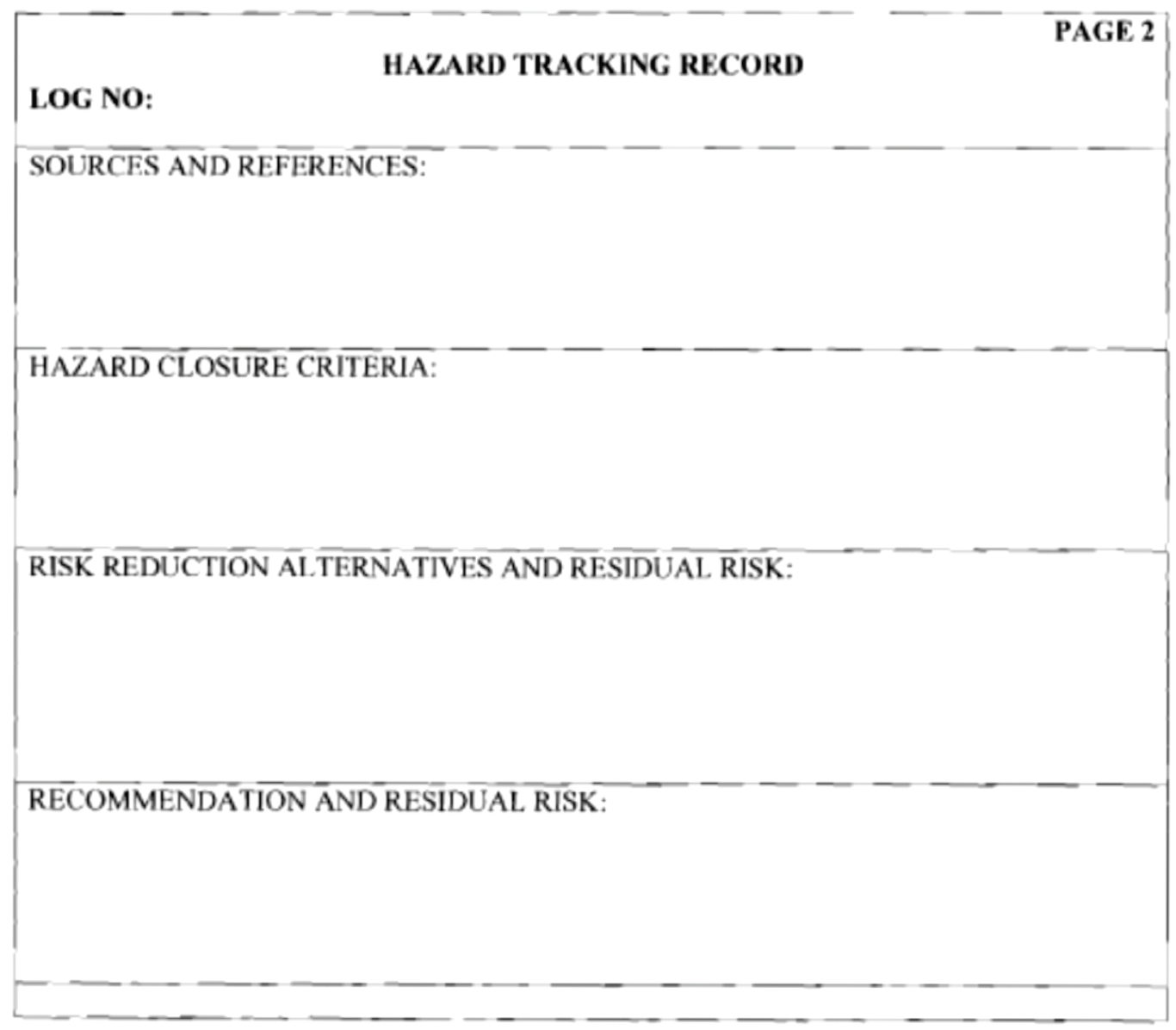




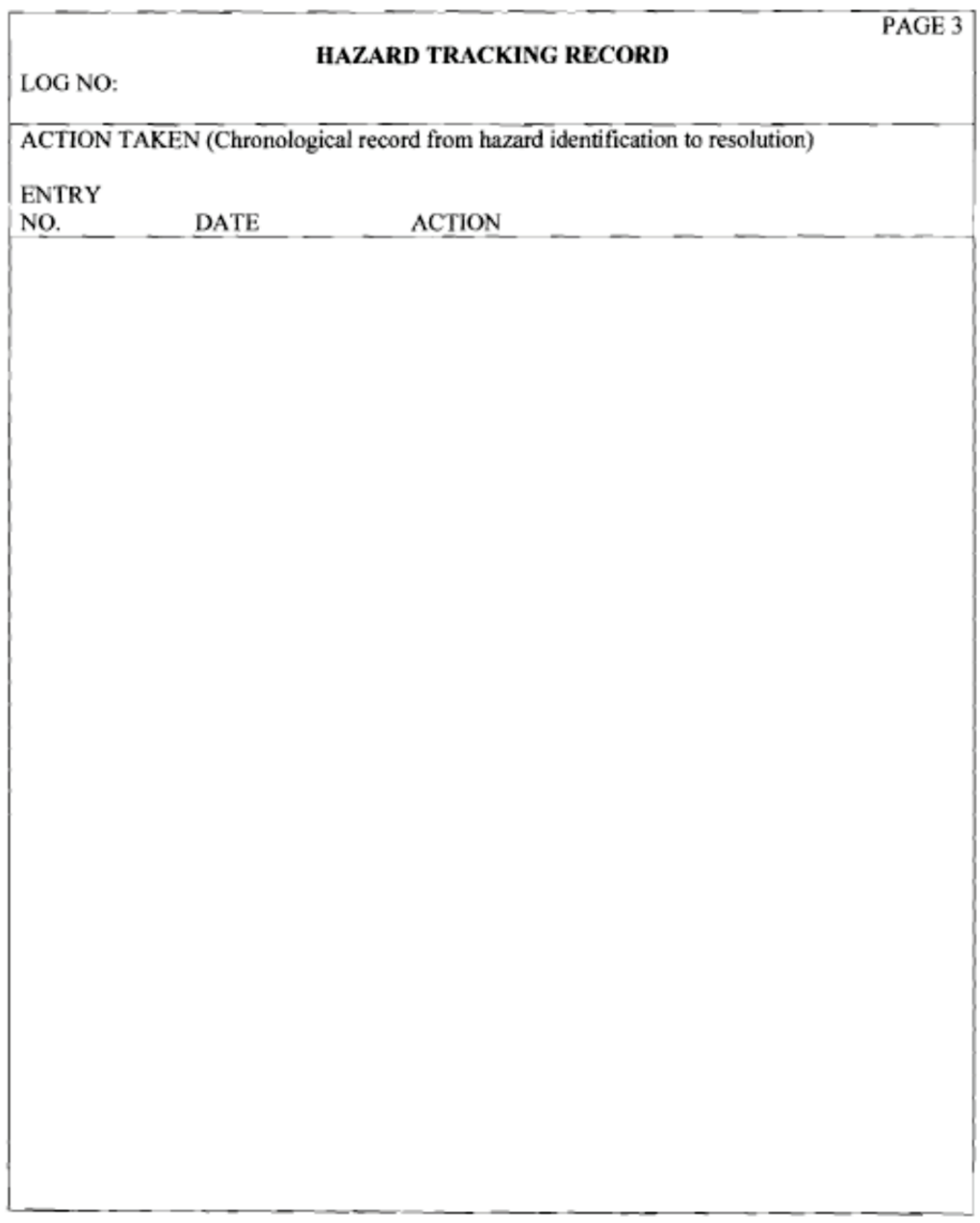

B-3 


\section{Appendix F: Operational Risk Assessment and Acceptance Memo Template (unformatted)}

The following template is reproduced here so that other users have knowledge of the contents of an Operational Risk Assessment and Acceptance memorandum. NOE: Highlighted text refers to individual operations. 


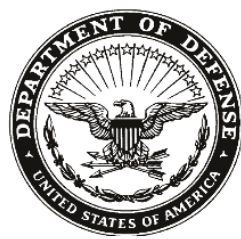

\section{DEPARTMENT OF THE ARMY US ARMY ADDRESS OF THE REQUESTING UNIT}

\section{OFFICE SYMBOL OF REQUESTING UNIT}

MEMORANDUM FOR: U.S. Army Engineering Directorate

SUBJECT: Operational Risk Assessment and Acceptance for XYZ UAS AND ABC OPERATIONAL AREA

1. Reference System Safety Management Plan for XYZ Unmanned Systems, DATE OF DOCUMENT SIGNATURE

2. The $A B C$ District $X Y Z$ Unmanned Aerial Vehicle System (UAS) Team has evaluated the composite residual risk for the $A B C O P E R A T I O N A L A R E A$ as a “(THE LEVEL OF RISK DETERMINED AND ACCEPTED, LOW, MEDIUM, $H I G H)$ " risk mission.

3. ABC District XYZ UAS TEAM was tasked to perform an Operation Risk Assessment based on the Level 3 Airworthiness issued by AED of the $A B C$ OPERATIONAL AREA for the XYZ UAS. The Level 3 Airworthiness Releases (AWRs) are considered as safety cases and as such non-traditional airworthiness issues are considered for each project site. The ABC District XYZ UAS TEAM is requesting that $\mathrm{AED}$ issue an $\mathrm{AWR}$ for the $A B C O P E R A T I O N A L A R E A$ /ocation

4. This Operational Risk Assessment was done to include the operational $X Y Z$ $U A S$ history and to determine operational risks involved at the site to be flown.

5. ABC District XYZ UAS TEAM notes that the size, weight, speed and duration of flight of the $X Y Z U A S$ resolve many airworthiness issues other small UAS engender. However because the system has not undergone extensive airworthiness qualification $X Y Z U A S$ must consider this system unreliable and subject to erroneous behaviour at any time during its operation and mitigate for potential hazards. The operational mission profile will focus on operational considerations (where and how to operate) to support the AWR safety case.

6. At this time, because of a lack of MIL-STD-810 test characterization $A B C D i s$ trict XYZ UAS TEAM finds hazards associated with this system are not quantifiable and therefore defaults to conducting flight operations in areas where personnel situational awareness of operation and population density will be controlled as mitigation. 
7. The undersigned acknowledges and accepts the (THE LEVEL OF RISK DETERMINED AND ACCEPTED, LOW, MEDIUM, HIGH) level risks associated with operation of the $X Y Z U A S$

Name

Rank/Title (e.g. MG, GS-15)

District or Unit Location

Branch of Army (e.g. United States Special Operations Command, USACE, etc.),

Encl 


\section{Appendix G: Material Risk Assessment Memo Template}

The following template is reproduced here so that other users have knowledge of the contents of a Material Risk Assessment memorandum. NOTE: Highlighted text refers to individual assessments. 


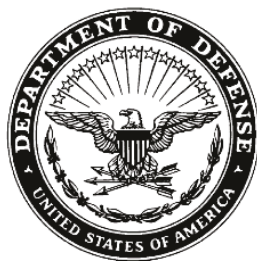

\section{DEPARTMENT OF THE ARMY \\ US ARMY ADDRESS OF THE REQUESTING UNIT}

OFFICE SYMBOL OF REQUESTING UNIT

MEMORANDUM FOR: U.S. Army Engineering Directorate (AED)

SUBJECT: Material Risk Assessment and Acceptance for XYZ UAS

1. Reference System Safety Management Plan for XYZ Unmanned Systems, DATE OF DOCUMENT SIGNATURE

2. The $A B C$ District $X Y Z$ Unmanned Aerial Vehicle System (UAS) Team has evaluated the composite residual risk for the XYZ Unmanned Systems as a "(THE LEVEL OF RISK DETERMINED AND ACCEPTED, LOW, MEDIUM, HIGH)" risk.

3. ABC District XYZ UAS TEAM performed a Material Risk Assessment based on the Airworthiness Technical Interchange Meeting (TIM) held by AED for the $X Y Z U A S$. AED concluded the $X Y Z U A S$ has not met full airworthiness qualification or reliability demonstration requirements. Evidence that the $X Y Z U A S$ was designed to accepted engineering standards and/or evidence of adequate engineering data to determine their compliance with acceptance standards was not presented at the TIM. The reliability and safety of this system cannot be determined therefore it must be considered not airworthy and subject to unintended behavior or catastrophic failure at any time during operations.

4. This Material Risk Assessment acknowledges AEDs findings. ABC District $X Y Z U A S T E A M$ notes that the size, weight, speed and duration of flight of the $X Y Z U A S$ resolve many airworthiness issues other small UAS engender. However, because the system has not undergone extensive airworthiness qualification $A B C$ District XYZ UAS TEAM must consider this system unreliable and subject to erroneous behaviour at any time during its operation and mitigate for potential hazards. The operational mission profile will focus on operational considerations (where and how to operate) to support the AWR safety case.

5. The undersigned acknowledges and accepts the (THE LEVEL OF RISK DETERMINED AND ACCEPTED, LOW, MEDIUM, HIGH) level risks associated with operation of the $X Y Z U A S$ 
Name

Rank/Title (e.g. MG, GS-15)

District or Unit Location

Branch of Army (e.g. United States Special Operations Command, USACE, etc.),

Encl 


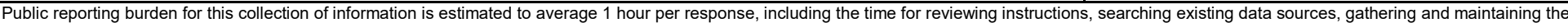

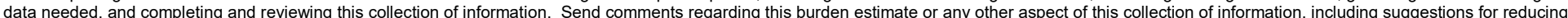

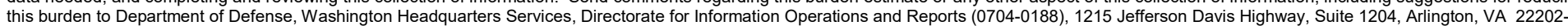

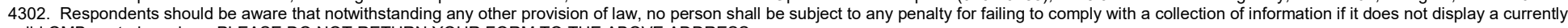
valid OMB control number. PLEASE DO NOT RETURN YOUR FORM TO THE ABOVE ADDRESS.
1. REPORT DATE (DD-MM-YYYY)
December 2018

4. TITLE AND SUBTITLE

Evaluation of Roof Leak Detection Utilizing Unmanned Aircraft Systems Equipped with

Thermographic Sensors
3. DATES COVERED (From - To)

5a. CONTRACT NUMBER

5b. GRANT NUMBER

5c. PROGRAM ELEMENT NUMBER

5d. PROJECT NUMBER

457492

5e. TASK NUMBER

5f. WORK UNIT NUMBER

8. PERFORMING ORGANIZATION REPORT NUMBER

ERDC/CERL TR-18-39

\section{PERFORMING ORGANIZATION NAME(S) AND ADDRESS(ES)}

U.S. Army Engineer Research and Development Center (ERDC)

Construction Engineering Research Laboratory (CERL)

PO Box 9005

Champaign, IL 61826-9005

\section{SPONSORING / MONITORING AGENCY NAME(S) AND ADDRESS(ES)}

Office of the Assistant Chief of Staff for

Installation Management

600 Army Pentagon

Washington, DC 20314-1000

\section{DISTRIBUTION / AVAILABILITY STATEMENT}

Approved for public release. Distribution is unlimited.

\section{SUPPLEMENTARY NOTES}

\section{ABSTRACT}

Management of the extensive U.S. Army roof portfolio increasingly re-lies on intelligent, data-driven decisions in a resource-limited environment. Roof leaks constitute millions of dollars in damage to equipment, insulation, and other assets, and can be the source for mold and other human environmental hazards. In recent years, thermographic imaging has been used as a nondestructive means to detect the location of wet insulation in low-pitched roofs. Traditional acquisition methods of non-destructive roof leak detection include handheld rooftop inspection and aerial imaging by conventional, manned aircraft. Through the advent and maturation of unmanned aircraft system (UAS) technology, nondestructive thermographic inspections are a viable alternative to tradition-al thermographic data acquisition methods. This report concludes that the use of a UAS for roof leak detection is a cost-effective and efficient alter-native to other inspection methods, and it gives recommendations for the U.S. Army's use of the technology.

\section{SUBJECT TERMS}

Drone aircraft, Remote sensing, Thermography, Roofing, Water leakage, Military bases

\begin{tabular}{|c|c|c|c|c|c|}
\hline \multicolumn{3}{|c|}{ 16. SECURITY CLASSIFICATION OF: } & \multirow{2}{*}{$\begin{array}{l}\text { 17. LIMITATION } \\
\text { OF ABSTRACT } \\
\text { UU }\end{array}$} & \multirow{2}{*}{$\begin{array}{l}\text { 18. NUMBER } \\
\text { OF PAGES } \\
123\end{array}$} & 19a. NAME OF RESPONSIBLE PERSON \\
\hline $\begin{array}{l}\text { a. REPORT } \\
\text { Unclassified }\end{array}$ & $\begin{array}{l}\text { b. ABSTRACT } \\
\text { Unclassified }\end{array}$ & $\begin{array}{l}\text { c. THIS PAGE } \\
\text { Unclassified }\end{array}$ & & & $\begin{array}{l}\text { 19b. TELEPHONE NUMBER (in- } \\
\text { clude area code) }\end{array}$ \\
\hline
\end{tabular}

